NASA-CR-200112

\title{
A Scientific Assessment of a New Technology Orbital Telescope
}




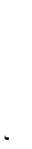




\section{A Scientific Assessment of a New Technology Orbital Telescope}

Task Group on BMDO New Technology Orbital Observatory

Space Studies Board

Commission on Physical Sciences, Mathematics, and Applications

National Research Council 
NOTICE: The project that is the subject of this report was approved by the Governing Board of the National Research Council, whose members are drawn from the councils of the National Academy of Sciences, the National Academy of Engineering, and the Institute of Medicine. The members of the task group responsible for the report were chosen for their special competences and with regard for appropriate balance.

This report has been reviewed by a group other than the authors according to procedures approved by a Report Review Committee consisting of members of the National Academy of Sciences, the National Academy of Engineering, and the Institute of Medicine.

The National Academy of Sciences is a private, nonprofit, self-perpetuating society of distinguished scholars engaged in scientific and engineering research, dedicated to the furtherance of science and technology and to their use for the general welfare. Upon the authority of the charter granted to it by the Congress in 1863 , the Academy has a mandate that requires it to advise the federal government on scientific and technical matters. Dr. Bruce M. Alberts is president of the National Academy of Sciences.

The National Academy of Engineering was established in 1964, under the charter of the National Academy of Sciences, as a parallel organization of outstanding engineers. It is autonomous in its administration and in the selection of its members, sharing with the National Academy of Sciences the responsibility for advising the federal government. The National Academy of Engineering also sponsors engineering programs aimed at meeting national needs, encourages education and research. and recognizes the superior achievements of engineers. Dr. Harold Liebowitz is president of the National Academy of Engineering.

The Institute of Medicine was established in 1970 by the National Academy of Sciences to secure the services of eminent members of appropriate professions in the examination of policy matters pertaining to the health of the public. The Institute acts under the responsibility given to the National Academy of Sciences by its congressional charter to be an adviser to the federal government and, upon its own initiative, to identify issues of medical care, research, and education. Dr. Kenneth I. Shine is president of the Institute of Medicine.

The National Research Council was organized by the National Academy of Sciences in 1916 to associate the broad community of science and technology with the Academy's purposes of furthering knowledge and advising the federal government. Functioning in accordance with general poljcies determined by the Academy, the Council has become the principal operating agency of both the National Academy of Sciences and the National Academy of Engineering in providing services to the government, the public, and the scientific and engineering communities. The Council is administered jointly by both Academies and the Institute of Medicine. Dr. Bruce M. Alberts and Dr. Harold Liebowitz are chairman and vice chairman, respectively, of the National Research Council.

Support for this project was provided by NASA Contract NASW 4627 and by Research Grant N00014-94-1-G031 between the National Academy of Sciences and the Department of the Navy, Office of the Chief of Naval Research. The content of this report does not necessarily reflect the position or the policy of the U.S. government, and no official endorsement should be inferred.

Copies of this report are available from

Space Studies Board

National Research Council

2101 Constitution Avenue, N.W.

Washington, D.C. 20418

Copyright 1995 by the National Academy of Sciences. All rights reserved.

Printed in the United States of America 


\section{TASK GROUP ON BMDO NEW TECHNOLOGY ORBITAL OBSERVATORY}

MICHAEL F. A'HEARN, University of Maryland, Chair

ROGER ANGEL, University of Arizona

ANITA COCHRAN, University of Texas, Austin

JAMES L. ELLIOT, Massachusetts Institute of Technology

HOLLAND C. FORD, ${ }^{*}$ Johns Hopkins University

CHRIST FTACLAS, Hughes Danbury Optical Systems

GARTH D. ILLINGWORTH, University of California, Santa Cruz

Staff

DAVID H. SMITH, Executive Secretary

ALTORIA B. ROSS, Senior Program Assistant

ERIN C. HATCH, Research Assistant

CHRISTOPHER DES AUTELS, Research Assistant

"Term ended in 1994, but continued as a liaison to the Space Telescope Science Institute. 


\section{SPACE STUDIES BOARD}

CLAUDE R. CANIZARES, Massachusetts Institute of Technology, Chair JOHN A. ARMSTRONG, IBM Corporation (retired)

LAWRENCE BOGORAD, Harvard University

JOSEPH A. BURNS, ${ }^{*}$ Cornell University

JOHN J. DONEGAN, U.S. Navy (retired)

ANTHONY W. ENGLAND, University of Michigan

DANIEL J. FINK, D.J. Fink Associates, Inc.

MARTIN E. GLICKSMAN, Rensselaer Polytechnic Institute

RONALD GREELEY, Arizona State University

BILL GREEN, former member, U.S. House of Representatives

HAROLD J. GUY, ' University of California, San Diego

NOEL W. HINNERS, Lockheed Martin Astronautics

JANET G. LUHMANN, University of California, Berkeley

JOHN H. McELROY, University of Texas, Arlington

ROBERTA BALSTAD MILLER, Consortium for International

Earth Sciences Information Network

BERRIEN MOORE III, University of New Hampshire

MARY JANE OSBORN, University of Connecticut Health Center

SIMON OSTRACH, Case Western Reserve University

CARLÉ M. PIETERS, Brown University

JUDITH PIPHER, " University of Rochester

MARCIA J. RIEKE, University of Arizona

ROLAND SCHMITT, Clifton Park, New York

JOHN A. SIMPSON, University of Chicago

ARTHUR B.C. WALKER, JR., ' Stanford University

MARC S. ALLEN, Director

*Former member. 
COMMISSION ON PHYSICAL SCIENCES, MATHEMATICS, AND APPLICATIONS

ROBERT J. HERMANN, United Technologies Corporation, Chair

STEPHEN L. ADLER, Institute for Advanced Study

PETER M. BANKS, Environmental Research Institute of Michigan

SYLVIA T. CEYER, Massachusetts Institute of Technology

L. LOUIS HEGEDUS, W.R. Grace and Co.

JOHN E. HOPCROFT, Cornell University

RHONDA J. HUGHES, Bryn Mawr College

SHIRLEY A. JACKSON, U.S. Nuclear Regulatory Commission

KENNETH I. KELLERMANN, National Radio Astronomy Observatory

KEN KENNEDY, Rice University

HANS MARK, University of Texas, Austin

THOMAS A. PRINCE, California Institute of Technology

JEROME SACKS, National Institute for Statistical Sciences

L.E. SCRIVEN, University of Minnesota

LEON T. SILVER, California Institute of Technology

CHARLES P. SLICHTER, University of Illinois at Urbana-Champaign

ALVIN W. TRIVELPIECE, Oak Ridge National Laboratory

SHMUEL WINOGRAD, IBM T.J. Watson Research Center

CHARLES A. ZRAKET, The MITRE Corporation (retired)

NORMAN METZGER, Executive Director 



\section{Preface}

During the first decade of its existence, the Strategic Defense Initiative Organization (now the Ballistic Missile Defense Organization-BMDO) spent approximately $\$ 25$ billion on development of new technology in support of its ballistic missile defense objectives. Apart from kinetic- and directed-energy weapons development, a significant fraction of these resources were devoted to development of technology for lightweight, high-performance spacecraft and their subsystems. With the Cold War at an end and a new emphasis on exploitation of defense-related technology in the civilian and even commercial sectors, the availability of innovative space capabilities is providing new opportunities for space research that have previously been precluded by cost, weight, or technical feasibility.

The application of defense technology is facilitated when space missions are designed in such a way as to simultaneously serve defense test needs and scientific data collection. An example of such an approach was the Clementine small spacecraft mission, launched in January 1994; its military goals were the verification of certain spacecraft subsystems under extended flight conditions in the deep-space radiation environment. The test program, however, was designed in consultation with the planetary science community. As a result, Clementine was able to collect valuable scientific data about the Moon-data that would not otherwise be available to the scientific community. NASA has now recognized the value of missions devoted to technology demonstrations by proposing the New Millennium program.

As part of a program designed to test the Alpha chemical laser weapons system in space, BMDO developed components of an agile, lightweight, 4-meter telescope, equipped with an advanced active-optics system. The original test program included both a tracking and a weapons component. Because of budget shortfalls and program redirection, however, BMDO abandoned space testing of the weapons component some years ago. More recently, BMDO was forced to defer the pointing and tracking portion of the test program.

BMDO had proposed to make space available in the telescope's focal plane for instrumentation optimized for scientific applications in astrophysics and planetary astronomy for a potential flight mission. Such a flight mission could still be undertaken if new or additional sponsorship can be found. Despite this uncertainty, BMDO requested assistance in defining the instrumentation and other design aspects necessary to enhance the scientific value of a pointing and tracking mission "should this program or a similar one be funded in a subsequent Defense Plan." In response to this request, the Space Studies Board established the Task Group on BMDO New Technology Orbital Observatory (TGBNTOO) and charged it to: 
1. Provide instrumentation, data management, and science-operations advice to BMDO to optimize the scientific value of a 4-meter mission; and

2. Support a Space Studies Board assessment of the relative scientific merit of the program.

This report deals with the first of these tasks.

The initial, information-gathering phase of the study involved site visits to Itek Optical Systems in Lexington, Massachusetts (October 6-7, 1994) and Lockheed Missiles and Space Co. in Sunnyvale, California (November $17-$ 19, 1994). During the course of these meetings, task group members were briefed on the 4-meter telescope project by representatives from BMDO, Charles Stark Draper Laboratories, and host organizations. While at Itek, members inspected completed optical components of the 4-meter telescope and viewed associated manufacturing and testing facilities. While at Lockheed, members toured spacecraft production and testing facilities. A detailed outline of this report and initial drafts of its sections were completed at the task group's third and final meeting, which was held in Washington, D.C. (December 19-20). Work on drafting and finalizing the report continued during the first half of 1995.

The TGBNTOO appreciates the time and thoughtful attention provided by the many individuals who helped contribute to this report. We wish to single out the following for special thanks: Sam Williams (Lockheed Missiles and Space Co.), Roland Plante (Itek Optical Systems), Larry Stepp (Gemini 8-meter Telescope Project), William Holzer (Hughes Santa Barbara Research Center), and, in particular, Stephen E. Strom (University of Massachusetts, Amherst), for his considerable input to the section on star formation in Chapter 5. The comments and criticisms of reviewers of early drafts of this report are gratefully acknowledged. Of course, the findings, conclusions, and judgments of this report are solely the responsibility of the task group.

Michael F, A'Hearn, Chair

Task Group on BMDO

New Technology Orbital

Observatory 


\section{Contents}

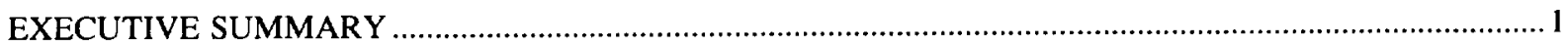

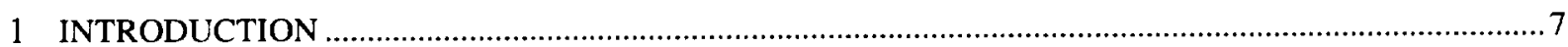

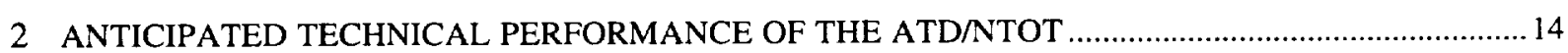

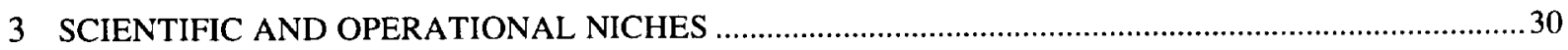

4 ASTRONOMICAL TECHNOLOGY DEMONSTRATIONS ….............................................................. 40

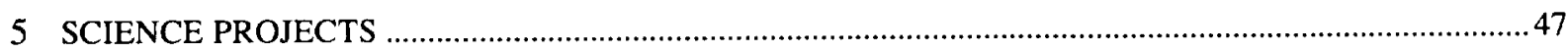

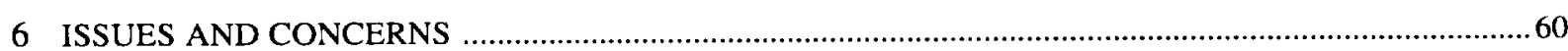

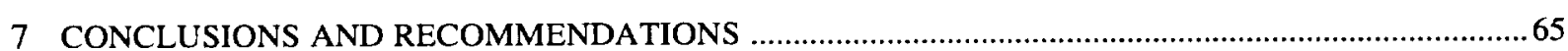

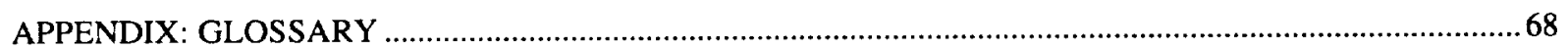





\section{Executive Summary}

The end of the Cold War and a decline in the fortunes for space research have gone hand in hand. If the space sciences are to continue advancing and not slip into a decline matching their dwindling budgets, new and innovative ways will have to be found to perform space missions. This is particularly true for space astronomy, given that certain features of spacecraft design (e.g., telescope apertures and detector sizes) are constrained by the laws of physics and cannot be miniaturized and still carry out their scientific tasks.

Not all of the events of recent years have been detrimental for space science. The decline in superpower rivalries has opened new avenues for international cooperation. Similarly, once-secret military technology has become available for civilian applications. Indeed, declining defense and space budgets have given rise to hybrid projects with both military and scientific goals. Prime among these was the recent Clementine lunar orbiter. This report assesses another such project, a large space telescope. In addition to demonstrating technology of interest to the Department of Defense (DOD), this mission has significant scientific capabilities, both in enabling direct astronomical observations and in demonstrating technology that may drastically alter the cost/performance ratio of future NASA missions.

At the height of the Cold War, the DOD's Strategic Defense Initiative Organization (SDIO) actively sponsored development of the technology needed to make space-based laser weapons feasible. As part of this program, SDIO developed many components of an agile, ultra-lightweight, 4-meter space telescope, equipped with an advanced active-optics system. Budgetary shortfalls and program redirection led to the cancellation of any tests of laser weapons in space. However, many components of the system had other applications of interest to both the DOD and the scientific community, and so development of the space-based telescope continued under the socalled Advanced Technology Demonstrator (ATD) program of SDIO's successor, the Ballistic Missile Defense Organization (BMDO).

To assist in the evaluation of the ATD's scientific potential, BMDO asked the Space Studies Board to provide advice on instrumentation, data management, and science operations to optimize the scientific value of a 4-meter mission. Following the initiation of the study by the Task Group on BMDO New Technology Orbital Observatory, however, a combination of budgetary pressures and redirected defense priorities forced BMDO to defer the ATD mission. Nevertheless, BMDO reaffirmed that "planning advice and recommendations [about the scientific aspects of the 4-meter mission] would still be valuable in formulating future joint experiments should this program or a similar one be funded in a subsequent Defense Plan."

Despite the uncertain future of a flight test of the 4-meter telescope and the currently unknown national 
security goals of such a mission, the task group proceeded to analyze the astronomical potential of the deferred mission. Given the potential scientific aspects of the 4-meter telescope, this project is referred to as the New Technology Orbital Telescope (NTOT), or as the ATD/NTOT, to emphasize its dual-use character. The task group emphasizes that it was specifically charged to assess the astronomical capability of the ATD/NTOT and therefore included only people with competence for that specific assessment.

The ATD/NTOT mission was conceived as a low-cost demonstration of technology, intended for use in future national security spacecraft, but having implications for astronomy. As such it is:

- Designed to cost ( $\$ 350$ million, including launch);

- Uses existing technology and/or designs wherever possible;

- Has a 3-year development schedule and a nominal orbital lifetime of 1 year; and

- Is not driven by specific astronomical requirements.

Given these characteristics, the task group adopted the basic philosophy that any potential involvement of the astronomical community in the ATD/NTOT should, at least initially, be predicated on the assumption that the ATD/NTOT is primarily a test of new technology for astronomy and is not a mission driven by any particular astronomical requirements. In this light, the ATD/NTOT's greatest benefit to the astronomical community will be to show whether or not it is possible to break the Hubble paradigm-that is, to demonstrate that it is possible to obtain large space optics at low cost. While doing this, it could carry out major astronomical studies not possible with the Hubble Space Telescope (HST) even with its currently planned improvements in instrumentation.

The basic features of the ATD/NTOT are the following:

- A 4-meter-aperture, 17-mm-thick, primary mirror equipped with some 260 actuators for on-orbit refiguring;

- Afocal optics with an image-stabilization mirror located at an image of the entrance pupil to adjust the pointing anywhere within a \pm 5.7 -arc-minute region without moving the spacecraft;

- Graphite polycyanate (graphite epoxy) structures for the entire telescope assembly;

- Use of an on-board inertial reference to maintain pointing stability over a bandwidth from 1 to $300 \mathrm{~Hz}$;

- The ability to track stars, as faint as 19 th magnitude, through the full aperture of the telescope to maintain pointing stability against disturbances at frequencies of less than about $10 \mathrm{~Hz}$;

- A design optimized for agility and rapid slewing from one part of the sky to another; and

- A highly eccentric orbit with a 12-hour period allowing continuous viewing of targets over much of the sky for periods up to about 8 hours.

Estimates of the performance of the ATD/NTOT suggest that it approaches the diffraction limit at nearinfrared wavelengths. In the optical, its full width at half maximum (FWHM) is better than that for any current or planned facility, while the diameter for 50\% encircled energy is comparable to the HST's. The reason for this is the relative roughness of the primary mirror. In fact, the primary mirror dominates all other sources of wavefront errors in the ATD/NTOT's error budget. This suggests an obvious enhancement: improving the figure of the primary mirror by a factor of two so that its contribution to the telescope-level error budget is comparable to that of the other components. Not only could this improvement be achieved at relatively low cost, but it would also have a dramatic impact on the ability of the ATD/NTOT to do both the technology demonstrations and the observing projects outlined in this report.

The baseline instrument package for the ATD/NTOT consists of a variety of optical- and infrared-array detectors. The one of most interest is a $1024 \times 1024$ indium antimonide (InSb) infrared array that would have state-of-the-art astronomical capabilities if operated at a cold enough temperature. The two passive, visible, finetracking arrays would have some astronomical applications. These would, however, be limited because their charge-coupled devices (CCDs) are line-transfer devices, and the arrays and their amplifiers are not optimized for low readout noise. The obvious deficiency in the instrument package is the absence of an optical framing camera of astronomical quality. The addition of such an instrument would have a very significant impact on the astronomical capabilities of the ATD/NTOT.

Understanding the areas in which the ATD/NTOT might have significant advantages over existing and 
planned facilities is critical to deciding which scientific projects and technological demonstrations to emphasize. To do this the task group considered two aspects of the ATD/NTOT: its optical and near-infrared performance, and the operational modes in which it can be used most cost effectively. Consideration of performance factors led to the conclusion that the ATD/NTOT has major advantages:

- In the near infrared (2 to 4 microns), where the sky background is reduced by several orders of magnitude;

- In the far red ( $>0.7$ micron), where the sky background is reduced by one order of magnitude;

- For programs that depend on high contrast between a point source and its neighborhood, or those that require subarc-second spatial resolution; and

- For programs that are photon-starved, that is, receive little attention with the HST.

Consideration of operational factors led to the conclusion that the ATD/NTOT is best suited to large surveys because repeated use of the telescope in a single mode, by a small team of scientists, is the most cost-effective operating procedure. In addition, large surveys make less than optimum use of complex, multiuser facilities such as the HST.

In determining what scientific and technological projects the ATD/NTOT is most suited to perform, the task group's overriding priority has been to minimize cost while still ensuring the capability to do exciting science. The first astronomical goal of an ATD/NTOT flight should be to test the applicability of its technology for use in future space science missions. Prime among these tests are (in no particular order):

- Demonstration by actual astronomical application of the ability to adequately refigure a large mirror in orbit to obtain astronomical-quality images, both with and without ground-based intervention;

- Evaluation of image quality and its consistency both across the field of view as the fast steering mirror stabilizes the telescope's line of sight and in a variety of thermal environments;

- Characterization of the likely degree of passive cooling by establishing the thermal emission from the optical and other components of the system in both the initial low Earth orbit and the eventual highly elliptical (or Molniya) orbit;

- Investigation of the stability of field distortions, particularly as the figure of the primary mirror responds to its control actuators and, also, as the fast-steering mirror stabilizes the telescope's line of sight;

- Exploitation of the ATD/NTOT's agility and large fuel reserves to actively maneuver the spacecraft so that it is in the right place, at the right time, to observe ephemeral events such as occultations;

- Exploration of the possibilities presented by the Molniya orbit to conduct very long integrations in a costeffective manner; and

- Utilization of the facility as an experimental testbed for various modes of ground operations that may be needed for future space science missions.

The implementation of some of the task group's suggested enhancements to the baseline ATD/NTOT would significantly improve the evaluation of both the technology and the astronomical significance of a program of technology demonstrations. Two of the most important enhancements are enhancing the figure of the primary mirror and adding an optical framing camera. These improvements would allow far more rigorous tests of, for example, the image quality that can be realized with the ATD/NTOT technology. Addition of just the optical framing camera would permit studies of the system's photometric stability. Also of great importance is enhancing the cooling of the InSb array, since this would allow a better evaluation of the ATD/NTOT's infrared performance.

If the ATD/NTOT's technology passes its key tests and can exceed its 1-year design lifetime, then it will have a significant capability for astronomical research. An area in which the ATD/NTOT should excel is in studies of origins. The creation and evolution of the universe and its component galaxies, stars, and planets is a topic of great scientific and popular interest and one in which large-scale surveys play major roles. To highlight the ATD/ NTOT's potential in these areas, the task group discusses four possible observing programs:

- A series of deep surveys of the early universe at near-infrared wavelengths to study the evolution of galaxies, define the magnitude/number-count relation, and search for new "standard candles" at high redshifts. All 
of these projects are consistent with the baseline mission but would benefit significantly from the addition of an optical framing camera and enhancement of the primary mirror.

- A survey of the outer solar system to define the size- and radial-distributions of the primitive bodies constituting the Kuiper Disk (down to $\sim 1-\mathrm{km}$ bodies at $\sim 40 \mathrm{AU}$ ). This project requires an optical framing camera and would benefit significantly from the use of an enhanced primary mirror.

- High-resolution optical studies of the disks, jets, and winds associated with young stellar objects during the embedded, accretion-dominated, and post-accretion phases of their evolution. The success of this project depends critically on enhancement of the primary mirror and the addition of an optical framing camera.

- Synoptic occultation observations of Pluto and Triton to monitor global atmospheric change due to seasonal variations in insolation. Although compatible with the baseline mission, this project may be expensive in terms of operations and use of spacecraft resources because it would involve extensive spacecraft maneuvering and orbital changes.

In the course of the task group's deliberations, a number of items arose that raise questions about the astronomical utility of the ATD/NTOT. In particular:

- Little or no systems analysis has been performed to verify that the ATD/NTOT's individual components can be combined to form a working astronomical telescope.

- There is some doubt about the ability of the baseline ATD/NTOT to track guide stars as faint as would be needed for certain observations. Although there are solutions to this problem, the problem may be moot if the telescope is devoted to surveys.

- Aspects of the ATD/NTOT's design, particularly that of the tripod supporting the secondary mirror, may scatter stray light into the focal plane.

- The rate at which the figure of the primary mirror will need correction as it deforms due to thermal and other drivers, and the impact corrections may have on observing overhead, are not clear.

- Software problems of the type that ultimately doomed the Clementine mission must be avoided.

- The effect of cosmic-ray events on the ATD/NTOT's imaging arrays when the spacecraft is operating in the highly eccentric Molniya orbit is a significant factor.

- Although building to cost is becoming a key feature of NASA's present and future missions, neither NASA nor the space science community has much experience with this mode of operation.

While the resolution of these issues is beyond the scope of this study, they must nevertheless be resolved as the ATD/NTOT mission is further defined.

The task group's analysis shows that the ATD/NTOT mission uses advanced technology that has important potential applications for future space astronomy missions. Furthermore, its advertised cost-effectiveness is crucial to NASA's ability to carry out significant space astronomy missions in an era of tightly constrained budgets. Both of these factors have not escaped the notice of other groups. Thus, both the High-Z and Polar Stratospheric Telescope concepts draw heavily on the capabilities of the ATD/NTOT's technology. The task group's basic conclusion is that the ATD/NTOT mission does have the potential for contributing in a major way to astronomical goals. It is equally clear that if the ATD/NTOT performs as advertised, it could undertake astronomical observations that could not be matched by any other facility now in existence or under development. Thus the task group's first and foremost recommendation is as follows:

1. To optimize the return to astronomy from the ATD/NTOT, the astronomical community should be directly involved in the continued study and development of this mission, including system engineering and complete mission analysis. These community representatives should be selected by NASA, and their role should be to advise NASA on the continuing value of this mission for astronomy. The group should include not only astronomers proposing specific observing programs, but also individuals with particular expertise in the design of large telescopes and space missions.

Since any scientific applications of the ATD/NTOT are a bonus, the task group further recommends the following: 
2. If the ATD/NTOT mission flies, a suite of tests of the suitability of its technology for astronomical applications should be carried out. Some of these tests can be conducted concurrently with DOD's demonstration mission, but others require an astronomical phase of the mission.

3. Although scientific goals must be kept in mind and accommodated insofar as possible during the planning of the ATD/NTOT mission, these goals should not impose requirements that would have a major impact on development or operations costs.

4. The ATD/NTOT's astronomical promise is sufficient, even at this preliminary stage, that it is appropriate to plan for a mission phase devoted to astronomical observations. The resources devoted to planning an astronomical mission should be kept to a minimum until such time as the ATD/NTOT's scientific and technological capabilities are better defined.

A mission phase dedicated to astronomical observations, while highly desirable, could be extremely expensive if not managed appropriately. Given the philosophy of designing to cost, the development costs for astronomical research programs must be kept to an absolute minimum. In order to minimize operational costs, the task group recommends the following:

5. The astronomical phase of the ATD/NTOT mission should be carried out by a principal investigator and a science team, with rotating membership to accommodate a range of scientific expertise. No provision should be made for a traditional guest observer program.

6. An extended ATD/NTOT mission should concentrate on extensive surveys that repeatedly use the ATD/ NTOT in a single mode.

7. Astronomical data collected in the ATD/NTOT mission should be delivered promptly to an existing public archive that is independent of and expected to outlive the mission.

Because DOD sponsorship of the ATD/NTOT is uncertain, the mission's exact specifications are unclear. The task group has assumed a baseline performance predicated on the requirements necessary to perform the mission that BMDO has now deferred. This analysis revealed several areas in which enhancements beyond the baseline specifications would have a significant impact on the ATD/NTOT's astronomical capabilities. Some of these enhancements may ultimately be required by, or at least be consistent with, a DOD mission if and when it is finally defined. Although the task group has not evaluated the cost-effectiveness of all of these enhancements (something that must be done during the system engineering phases of the mission), it has discussed their potential impact with representatives of Lockheed and Itek. In one case the costs are well defined and the performance benefits reasonably determined. In other cases, neither the costs nor the actual improvements in performance are very well determined. It is, however, the sense of this task group that these enhancements are likely to be very cost-effective and important for the astronomical aspects of the mission. In particular:

8. The figure of the ATD/NTOT's primary mirror should be improved by roughly a factor of two to reduce its surface error to $\sim 17 \mathrm{~nm}$, and, thus, the total system's wavefront error to roughly $50 \mathrm{~nm}$ rms. Itek estimates that this enhancement would cost $\sim \$ 100,000$.

9. A large-format, framing, optical CCD of astronomical quality should be included in the ATD/NTOT's focal-plane package. A less expensive but clearly less desirable option would be to replace the baseline linetransfer CCDs in the fine-tracking sensors with frame-transfer CCDs.

10. The ATD/NTOT's baseline infrared detector, an InSb array, should be optimized for sensitivity by, for example, additional cooling and by minimizing the number of emitting surfaces in the optical path.

The task group briefly discussed other enhancements that could significantly improve the scientific return from an extended mission dedicated to astronomical observations. Unlike those discussed above, however, all of these modifications would add significantly to the cost of the mission and/or perhaps be incompatible with the national security objectives of the mission. These enhancements include:

- Modifying the ATD/NTOT's orbit to minimize and/or stabilize the thermal load on the spacecraft;

- Optimizing the design to enhance the passive-cooling characteristics of the telescope and focal-plane instruments; and

- Adding additional instruments (e.g., a dedicated infrared focal plane) for scientific research. 
Even if the ATD/NTOT mission is not eventually funded by BMDO and does not find another sponsor in the national security community, the task group believes that the time devoted to this study has been of use. While the complete package of technologies embodied in the ATD/NTOT proposal promises exciting advances in astronomical capabilities, it should be remembered also that much of its hardware already exists. A complete adaptiveoptics system and examples of thin primary mirrors, for instance, currently sit gathering dust in testing chambers. Many of these subsystems are themselves interesting additions to the tools at the disposal of astronomers and may find scientific applications very different from those for which they were designed. The ATD/NTOT may never fly, but if this report does nothing more than illuminate some of the capabilities lurking in the shadows of the Cold War, it will have achieved something worthwhile.

\section{REFERENCE}

1. Payton, Gary E., Deputy for Technology Readiness, BMDO, memorandum to the Space Studies Board, October $24,1994$. 


\section{Introduction}

For decades astronomers have known about the advantages of locating astronomical telescopes in space. From this vantage point it is possible to view the full span of the electromagnetic spectrum. Images of astronomical bodies are not distorted by passage through Earth's turbulent atmosphere, and, in principle, observations are not limited by vagaries of the weather or the diurnal cycle.

The Hubble Space Telescope (HST) has vindicated many of these claims, but only at a very high cost. In the 15 years since the HST was built, enormous advances have been realized in the design, manufacturing, and testing of optical systems. These new technologies allow us to make realistic plans for the next-generation of space telescopes-spacecraft with optical systems that are larger and lighter, yet much cheaper, than the HST's.

New techniques for figuring and testing allow rapid and inexpensive polishing of large mirrors to accuracies rivaling or exceeding the HST's. The feasibility of using wavefront sensors and active-optics systems to control the figure of mirror surfaces has been proven by many groups. The University of Arizona and the European Southern Observatory, for example, have demonstrated that 4-meter-class mirrors, equipped with multiple actuators, can achieve wavefront errors of better than $53 \mathrm{~nm}$ rms (admittedly with substantially more rigid mirrors than those discussed in this report). A necessary component of such systems, the optical sensors used to construct wavefront maps from star measurements, has also been developed and proven by researchers on both sides of the Atlantic Ocean.

To be significantly less costly than the HST, a second-generation space telescope not only must employ many of the technologies outlined above, but also must utilize new operational concepts to reduce operating expenses. The use of a very thin, actively supported primary mirror, for example, can significantly reduce spacecraft mass. Similarly, placing the telescope in a high Earth orbit can significantly increase observing efficiency and greatly reduce demanding operational constraints relative to those of a similar instrument in a low Earth orbit, such as the HST.

Each of these new concepts, however, carries a significant penalty in terms of increased risk. And, neither NASA nor the astronomical community can afford another expensive and highly visible failure such as the HST's spherical aberration. Before it rushes to embrace these new technologies, the astronomical community must have some confidence that mission-critical elements will work as advertised. This report discusses the astronomical potential of a military project, which, it is claimed by its proponents, will provide astronomers with this technology validation at a relatively low cost.

Although this so-called Advanced Technology Demonstrator (ATD) mission is motivated by future national 
security requirements, the task group notes that many of its components and the technologies they embody are similar to those required for an advanced astronomical observatory in space. Thus the ATD hardware may potentially have a role to play as a New Technology Orbital Telescope (NTOT) in fields such as cosmology, astrophysics, and planetary astronomy. Given the dual nature of the mission, the task group henceforth refers to a joint military/astronomy mission exploiting the ATD hardware as the ATD/NTOT. A purely military mission is referred to as the ATD.

\section{BMDO'S ADVANCED TECHNOLOGY DEMONSTRATOR}

With funding from the Strategic Defense Initiative Organization (SDIO), Itek Optical Systems has applied many of the techniques relevant to future space telescopes to create a fully active 4-meter mirror system with a very thin facesheet and lightweight carbon-fiber support structure. It has been proposed that Itek's optics and other hardware be test flown as part of the ATD program sponsored by the Ballistic Missile Defense Organization (BMDO).

The basic philosophy adopted for the as yet unfunded ATD program is not to design hardware to meet requirements set by specific operational (or scientific) objectives. Rather, the ATD is a design-to-cost program in which the specific tasks to be accomplished are designed around the capabilities of existing or minimally modified hardware. Since these components are key to any potential scientific utility, the bulk of this report is devoted to assessing the ability of this hardware to perform a variety of priority astronomical observations.

As presented to the Task Group on BMDO New Technology Orbital Observatory by representatives of BMDO, Lockheed, and Itek, DOD's technological goals for the ATD program include the following: ${ }^{2}$

- Complete resolution of the ability to track and point a laser beam at a missile in the boosting phase in a space

environment from the correct orbit geometries and ranges for a space-based laser;

- Track and gather data on reentry vehicles and decoys at long ranges;

- Gather imagery in the visible and near-infrared of space objects at geosynchronous orbits;

- Gather Earth background data at high resolution on the water and carbon dioxide bands in the short- and medium-wave infrared as a data base for future system design;

- Gather data on the observables of a missile in the boost phase; and

- Demonstrate the ability to designate targets on the ground from a space platform for the use of laser-guided weapons in a tactical scenario.

The task group emphasizes that it has neither the charge nor the expertise to assess the utility, realism, or feasibility of these technology goals.

The principal components that must be assembled to undertake such tests are the following:

1. A laser;

2. A telescope;

3. A sensor package to aim at, to track, and to observe targets;

4. An image stabilization system to enable tracking of targets;

5. A spacecraft bus to provide housekeeping functions such as power and communications; and

6. A launch system to place the whole package into an appropriate orbit.

\section{The Telescope}

Perhaps the most important element of the ATD is its telescope. Since 1975, the federal government has invested more than $\$ 100$ million to fund a series of research and development programs at Itek aimed at the design, production, and testing of large, active-optics technology (see Box 1.1, "Optical Heritage," for more details). Although the ATD is designed to collimate and direct a high-energy laser beam, the changing world situation now means that it is possible to contemplate nonmilitary applications for the large, lightweight, segmented mirrors developed by these programs. 


\section{Box 1.1 Optical Heritage}

The optical components proposed for use in the ATD/NTOT draw on the heritage of a number of projects, including the Large Advanced Mirror Program of the 1980s and the Adaptive Large Optics Technologies and Large Optical Segment programs of the early 1990 s.

\section{Large Advanced Mirror Program}

The Large Advanced Mirror Program (LAMP) was a ground demonstration of a 4-meter, actively controlled, segmented mirror. Each of its seven, 2-meter, quasi-hexagonal segments was approximately $17 \mathrm{~mm}$ thick and attached to surface control actuators mounted in a stiff, lightweight graphite-epoxy back-up structure. A total of 312 surface control and 42 segment position actuators enabled the LAMP mirror to be controlled so as to yield a wavefront accuracy of better than $100 \mathrm{~nm}$ rms. The LAMP mirror was subsequently installed as the beam expander for a hydrogen fluoride laser at the San Juan Capistrano test range in California.

\section{Adaptive Large Optics Technologies}

The Adaptive Large Optics Technologies (ALOT) project-a 4-meter, lightweight, segmented-mirror telescope-was designed and built by ltek to test the technology for autonomous capture, phasing, and figure control of a fully adaptive primary mirror by sensing an extended scene in real time. The telescope consists of a central 2.6-meter annular mirror, with 144 actuators, surrounded by a number of $0.7 \times 2.1$-meter radial mirror segments (only one, however, was built) each equipped with 43 actuators (see Figure 1.1). Testing of ALOT in Itek's thermal-vacuum chamber revealed that the active control system could yield a residual wavefront error of $70 \mathrm{~nm}$ rms in the presence of active disturbances to the beam.

All of ALOT's support structures are made of graphite-epoxy composite, including the tripod supporting the secondary mirror, the Cassegrain baffle, and the reaction structure supporting the segmented primary mirror. All components, except the control electronics, are space qualifiable and compatible with launch by a Titan IV booster. The principal modifications required for space qualification of the existing ALOT hardware would be upgrading the electronics, replacing the control computer with one that is space-qualified, modifying the software, and adding thermal insulation and an external shroud.

The ATD/NTOT would utilize (or copy) much of the existing ALOT hardware with the exception of its segmented mirror.

\section{Large Optical Segment}

The Large Optical Segment (LOS) project is currently in progress. Its goal is to use the technology developed for LAMP to build two of the thirteen 4-meter segments required for an 11-meter mirror. Unlike the quasi-hexagonal structure of LAMP's primary, LOS's will resemble ALOT's in that it will consist of a central annular mirror surrounded by a number of radial segments. Each of the segments is ground from a 4-meter blank of Corning ULE glass.

The ATD/NTOT would substitute LOS's 4-meter monolithic central segment (or a copy) for ALOT's segmented mirror, thus greatly reducing the number of actuators needed to control the figure of the ATD/NTOT's primary mirror.

\section{Sensor Package}

The same military desires that spawned research into the means to control the targeting of space lasers also inspired research into the design of the instrumentation necessary to assess potential targets and aim weapons. One such activity was the StarLITE Special Studies Task, undertaken by Lockheed, ${ }^{2}$ which resulted in the design of a low-cost sensor suite, containing a variety of visible and infrared focal-plane arrays, that could acquire, track, and point a simulated laser weapon at a boosting target.

\section{Image Stabilization}

Tracking a boosting rocket at a distance of many hundreds to thousands of kilometers requires that the beam expander maintain a very precise line-of-sight stability. This stability may be achieved by the inclusion of an appropriate on-board inertial reference in the pointing and tracking system. An example of such a device is the Inertial Pseudo Stellar Reference Unit (IPSRU) developed at the Charles Stark Draper Laboratories. 


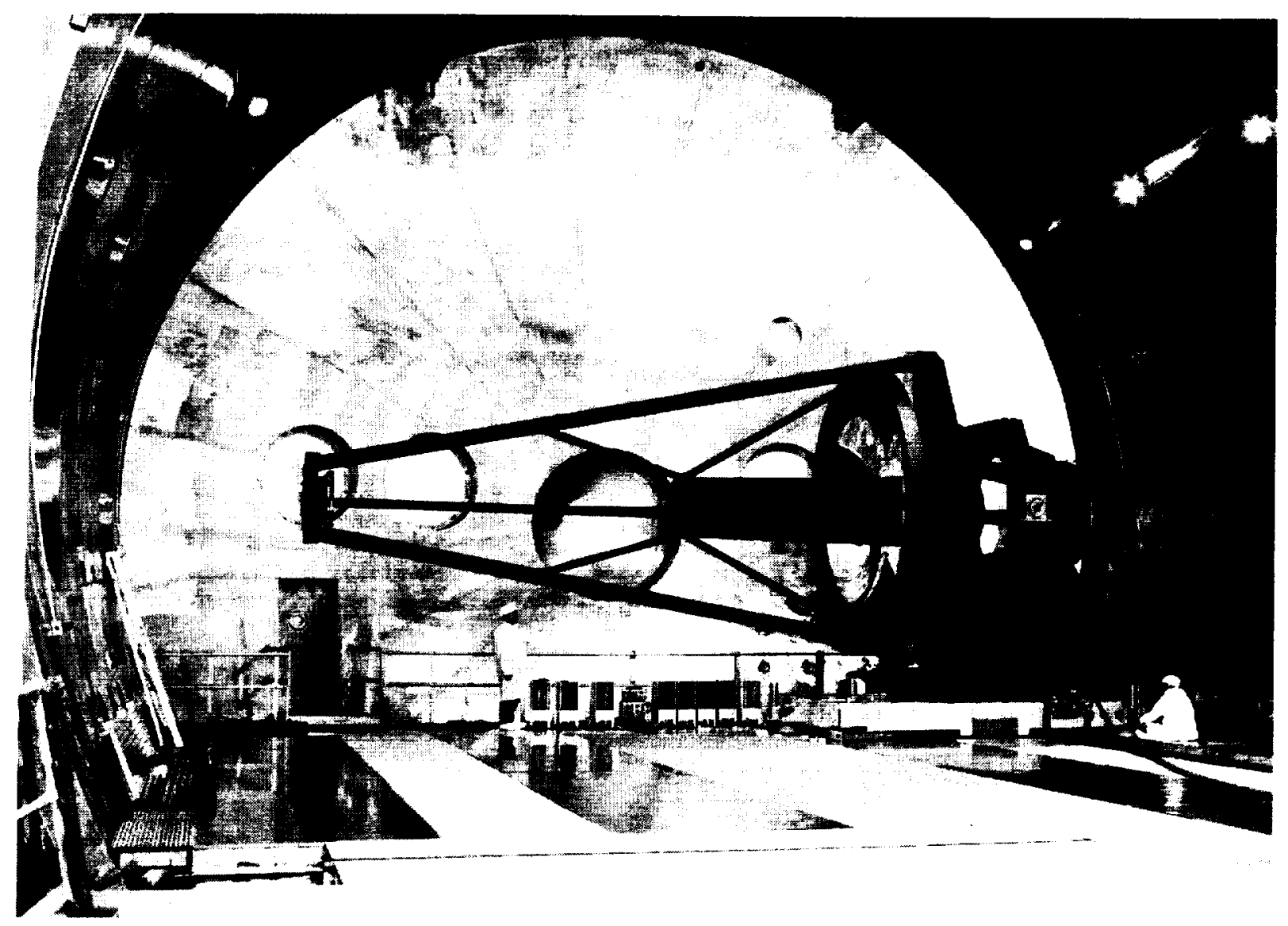

FIGURE 1.1 The 4-meter Advanced Large Optics Technologies (ALOT) telescope is shown in a testing chamber at Itek Optical Systems. The proposed ATD/NTOT project would reuse or replicate much of this hardware, with the exception of ALOT's 4-meter segmented mirror, which would be replaced by a monolithic, meniscus mirror of equal size similar to the central, annular segment of the Large Optical Segment project. (Courtesy of Itek.)

\section{Spacecraft Bus}

Custom-designing satellites to meet the requirements of each particular payload is inherently expensive. Commercial opportunities, such as meeting the demand for the many tens or hundreds of satellites required by, for example, global cellular telecommunications systems such as Iridium and Teledesic, have prompted many aerospace concerns to develop generic buses capable of supporting a variety of payloads. Lockheed's F-SAT (Frugal satellite), for example, can provide power, attitude control, computing, communications, and propulsion for payloads in the 1 - to 5 -ton class.

\section{Launch System}

The decline in superpower rivalry that has made once-classified defense technology available for civilian use has also opened other possibilities. Among these is access to Russia's stable of large, reliable, and relatively cheap booster rockets. Indeed, access to the highly capable Proton launcher is now marketed commercially by a consortium including Lockheed and several Russian enterprises. Two launches have been booked to date, with options taken out for an additional 13. The first flight is scheduled for March 1996. 
The proposed costs for the major components of the ATD, together with their construction schedules, are summarized in Box 1.2.

\section{POTENTIAL VALUE OF THE ATD/NTOT TO SPACE ASTRONOMY}

At first glance, the ATD/NTOT promises to be an evolutionary step in advancing technology in exactly the directions needed to eventually undertake some of the most challenging missions in space astronomy. One of the most fundamental questions is whether life exists elsewhere in the universe. An infrared space telescope capable of detecting Earth-like planets around nearby stars requires new technology such as that to be demonstrated by the proposed ATD/NTOT mission.

One possible design for an instrument to detect extrasolar planets would use two cooled mirrors, configured as a Bracewell interferometer to cancel the light of the star. Once a planet is detected, the same telescope could search the infrared spectrum of the planet's atmosphere for the presence of water, carbon dioxide, and ozone. Ozone at the same high level as seen in Earth's spectrum would be a strong indicator of life, since ozone is formed from oxygen that is created and maintained only by living organisms.

Other fundamental observations, such as the imaging of galaxies from their very beginnings early in the life of the universe, also require large cooled telescopes. The High-Z proposal, for example, developed in response to a 1994 request from NASA for new mission concepts in astrophysics, would attack this problem by deploying an instrument like the ATD/NTOT in a heliocentric orbit ranging from 1 to $3 \mathrm{AU} .{ }^{3}$ The natural background at a wavelength of 3 microns is, from this vantage, the darkest of any part of the optical/infrared spectrum. Thus, High$\mathrm{Z}$ could observe the distant universe, as it was near the beginning of time, with greater sensitivity than any other telescope. A somewhat similar concept, the Polar Stratospheric Telescope, envisages deploying a telescope similar to the ATD/NTOT beneath a high-altitude aerostat tethered in Alaska. ${ }^{4}$ The ATD/NTOT technology is

\section{Box 1.2 ATD Costs and Schedule}

The proposed costs of major elements of the ATD 4-meter space telescope, together with a summary of its construction schedule and the facilities to be used in its testing, are based on information supplied by Lockheed and Itek. Costs of modifications necessary for an astronomy mission are not included. Although the task group did not perform any detailed analyses to verify this information, nothing quoted below seems unreasonable.

Costs

- 4-meter telescope from ltek

$\sim 50 \mathrm{M}$

- Sensor package from Lockheed

$\sim 25 \mathrm{M}$

- F-SAT bus

- Payload integration

$-\$ 50 M$

$-\$ 40 M$

- Space vehicle integration

$-\$ 20 M$

- Proton launch vehicle

$-\$ 60 M$

- Space operations and data reduction $\quad-\$ 25 \mathrm{M}$

- Cost

- Management reserve

$\sim \$ 270 \mathrm{M}$

$-\$ 80 \mathrm{M}$

- Realistic costs

$\sim \$ 350 \mathrm{M}$

Schedule

- Itek can build 4-meter telescope in 2 years

- F-SAT bus available in 2 years

- Sensor suite also takes 2 years

- Modular approach allows for 12-month integration and testing

Available Test Facilities

- Itek's test chamber for telescope and sensor suite

- Lockheed's acoustic and thermal vacuum chambers for vehicle test 
even being considered for moderate-sized, ground-based telescopes, although the problems implicit in this application are quite different from those found in space applications, for example, the reasonably high-frequency response necessary to maintain the shape of the primary mirror in the presence of wind buffeting.

\section{TASK GROUP'S EXPERTISE, PHILOSOPHY, AND APPROACH}

Because it includes people with considerable expertise in the design, construction, and testing of astronomical optics, the task group can make at least reasonable estimates of the likelihood that the ATD/NTOT will achieve the optical performance advertised by its designers. Given this fact and the task group's charge most of this report is devoted to an analysis of the optical performance and potential of the ATD/NTOT. This focus is most appropriate because the optical components are the key features that allow the ATD/NTOT to be considered as a potentially viable astronomical project.

On the other hand, this task group has little or no experience in areas such as spacecraft engineering and structural analysis. Thus, it claims no expertise in judging the likelihood of success in these areas, even though such success is clearly crucial to providing a facility that is astronomically useful for the long term. Yet Lockheed has an extensive and successful record in these areas. The task group has therefore relied, for the most part, on the past performance achieved by Itek and Lockheed in producing many components of the system and on its own estimates of the compounding of various sources of errors and the extent to which they may affect the astronomical performance.

\section{Operating Philosophy}

The basic purpose of the ATD is to demonstrate technologies that may be used in future systems built by DOD. This approach has the advantage that critical components can be tested and proven in a realistic environment before any commitment is made to the development or deployment of inherently expensive systems. In this spirit, the task group adopted as its basic philosophy that any potential involvement of the astronomical community in the ATD/NTOT should, at least initially, be predicated on the assumption that the ATD/NTOT is primarily a test of new technology for astronomy and is not a mission driven by any particular scientific requirements.

In this light, the greatest value of the ATD/NTOT for astronomy will be to show whether or not it is possible to break the Hubble paradigm, that is, to demonstrate that it is possible to get large space optics at low cost. While doing so, it may also be able to contribute to major astronomical results.

\section{Attention to Possible Mission Enhancements}

Although the task group's approach is to evaluate what important scientific observations and tests can be performed during the ATD/NTOT's baseline mission (as it understands that mission), it notes that there is significant uncertainty as to the precise nature of the baseline mission. Discussions between the task group and project officials have already led to changes that either enhance or do not interfere with the baseline mission and do not have a significant cost impact. Accordingly, the task group also offers its thoughts on possible scientific enhancements to the mission, where appropriate. As the mission's goals evolve, some of these enhancements may add significantly to the value of the ATD/NTOT for all potential participants. Indeed, the principal purpose of involving astronomers early in the planning for the ATD/NTOT was to ensure that these discussions would be initiated and would continue.

\section{Content of This Report}

In the following chapters the task group examines the ATD/NTOT concept in more detail to determine if the ATD/NTOT is capable of performing useful astronomical observations. More importantly, it investigates whether the ATD/NTOT is an effective stepping-stone to the next generation of space telescopes. 


\section{REFERENCES}

1. An Advanced Technology Demonstrator for a 4-Meter Space Experiment, LMSC F439223, Lockheed Missiles \& Space Company, Sunnyvale, Calif., 1993.

2. ADAPT: StarLITE Special Studies Task Final Report, LMSC P005092, Lockheed Missiles \& Space Company, Sunnyvale, Calif., 1993.

3. Stockman, Hervey (Peter) S., High-Z: A Near-IR Space Telescope for Probing the Early Universe, Space Telescope Science Institute, Baltimore, Md., 1994.

4. Ford, H., Bely, P., Bally, J., Crocker, J., Dopita, M., Tilley, J., White, R., Allen, R., Bartko, F., Brown, R., Burg, R., Burrows, C., Clampin, M., Harper, D., Illingworth, G., McCray, R., Meyer, S., Mold, J., and Norman, C., "POST: A Polar Stratospheric Telescope," SPIE. Vol. 2199, Advanced Technology Optical Telescopes V, p. 298, 1994. (Note, this reference describes an early version of the POST concept.) 


\section{Anticipated Technical Performance of the ATD/NTOT}

The proposed ATD/NTOT is not a typical scientific mission in which technical specifications, derived from top-level science requirements, must be met during development, construction, and deployment. Rather, it is a mission designed to demonstrate newly available technologies that may be used in future DOD missions and that have obvious applications to scientific missions. As such, its performance must be characterized differently since it will be assembled, as much as possible, from existing components and designs rather than according to the specific requirements for a particular task. In this respect, the ATD/NTOT has more in common with the recent Clementine lunar orbiter than, say, the Hubble Space Telescope.

This chapter discusses the baseline concept for the ATD/NTOT as described by representatives of Itek and Lockheed. The technical evaluation of the mission is based on the task group's assessment of those aspects of the ATD/NTOT whose performance can be approximately predicted on the basis of these briefings. In other words, the task group describes the performance levels that the ATD/NTOT is most likely to achieve. The actual performance will be defined more clearly as launch approaches, although still not perfectly, given the nature of any technology demonstration. For this reason, the task group considers it essential that the astronomical community view the ATD/NTOT mission primarily as a demonstration and test of astronomically important technology, with significant scientific results being a hoped for, but not necessarily guaranteed, bonus. Recommended deviations from the baseline, where they significantly enhance astronomical performance, are identified in the course of this analysis.

\section{BASIC DESIGN FEATURES}

The telescope is the most fundamental element in determining the value of the ATD/NTOT mission for astronomy. Some of its key features have not been used previously in any astronomical telescopes in space. These features include:

- A 4-meter-aperture, thin-facesheet, primary mirror equipped with some 260 position actuators for on-orbit refiguring;

- Afocal optics with an image-stabilization mirror located at an image of the entrance pupil to adjust the pointing anywhere within a \pm 5.7 -arc-minute region without moving the spacecraft;

- Graphite polycyanate (graphite epoxy) structures for the entire telescope assembly; 
- Use of an on-board inertial reference to maintain pointing stability over a bandwidth from 1 to $300 \mathrm{~Hz}$;

- The ability to track stars, as faint as 19 th magnitude, through the full aperture of the telescope to maintain pointing stability against disturbances at frequencies of less than $10 \mathrm{~Hz}$; and

- A design optimized for agility and rapid slewing from one point on the sky to another.

The layout of the proposed telescope and details of its optics are shown in Figures 2.1 and 2.2, respectively. Its basic design is that of an afocal, three-mirror anastigmat. The use of three mirrors permits good imaging performance over a relatively large field of view. The 4-meter-diameter, $f / 1.25$ (a design with a focal ratio of $f / 1.7$ is also possible) parabolic primary mirror is $17 \mathrm{~mm}$ thick and is equipped with some 260 actuators to control its shape. The convex secondary and concave tertiary mirrors are both hyperboloids. The optical parameters of all three mirrors are given in Table 2.1.

The telescope relays an image of the entrance pupil from the primary mirror to a flat image-stabilization or fast steering mirror (FSM) with a demagnification of 40 (i.e., with an exit pupil diameter of $10 \mathrm{~cm}$ ). (A demagnification of 20 is another possibility.) The FSM, located at the relayed pupil, is used to stabilize the image at frequencies up to $300 \mathrm{~Hz}$ over an area of sky 0.2 degrees across.

The FSM is followed by a dichroic beam splitter that divides the light into infrared and visible branches at a wavelength near 1 micron. These separate beams are subsequently directed to a number of different focal planes by additional beam dividers and reimaging optics. The visible branch contains a wavefront sensor used to assess the shape of the primary mirror and generate correcting signals for the primary's figure control actuators. The assumed parameters of the completed telescope are summarized in Boxes 2.1 and 2.2 These numbers are used in subsequent sections that address the ATD/NTOT's optical performance.

\section{EXPECTED POINT-SPREAD FUNCTION}

Knowledge of the observatory-level point-spread function (PSF) and encircled energy is useful for defining the kinds of science and the spectral region to which an optical system such as the ATD/NTOT is best applied. In practice, of course, this determination is a complex systems engineering issue requiring many kinds of input and much analysis. However, the PSF can be estimated by considering two factors known about the ATD/NTOT: its line-of-sight stability and its figure error.

\section{Line-of-Sight Stability}

The telescope's line of sight is maintained at frequencies greater than $1 \mathrm{~Hz}$ by illuminating a quad cell with a gyroscopically stabilized light beam from a so-called Inertial Pseudo-Stellar Reference Unit (IPSRU). The resulting error signal is sampled at $300 \mathrm{~Hz}$ and used to drive the FSM so that it keeps the telescope's image fixed relative to the focal plane. Although the IPSRU's gyros drift at a rate of the order of 5 milliarc sec (mas) per second, they are corrected at frequencies of less than $10 \mathrm{~Hz}$ by determining the centroid of the image of a guide star viewed with the fine-guidance CCDs. The error-budget allotment for image stability is quoted as 4.5 mas per axis; thus, the two-dimensional image stability is 6.5 mas. The task group convolved this figure with a Gaussian to broaden expected point-spread functions.

\section{Figure Error}

For this discussion, the ATD/NTOT telescope is assumed to consist of five mirrors. The first three are the primary, secondary, and tertiary mirrors of the afocal telescope assembly. The fourth is the FSM at the reimaged telescope pupil, and the fifth is a hypothetical mirror required to bring light to a focus. Bringing the telescope to focus over a reasonable field of view actually requires at least two optics, but assuming only one makes only a small difference in predicted performance. Since astronomical telescopes generally feed a focused image to their instruments, the inclusion of the FSM and a focusing optic allows a direct comparison with performance at the focal plane of other telescopes. 

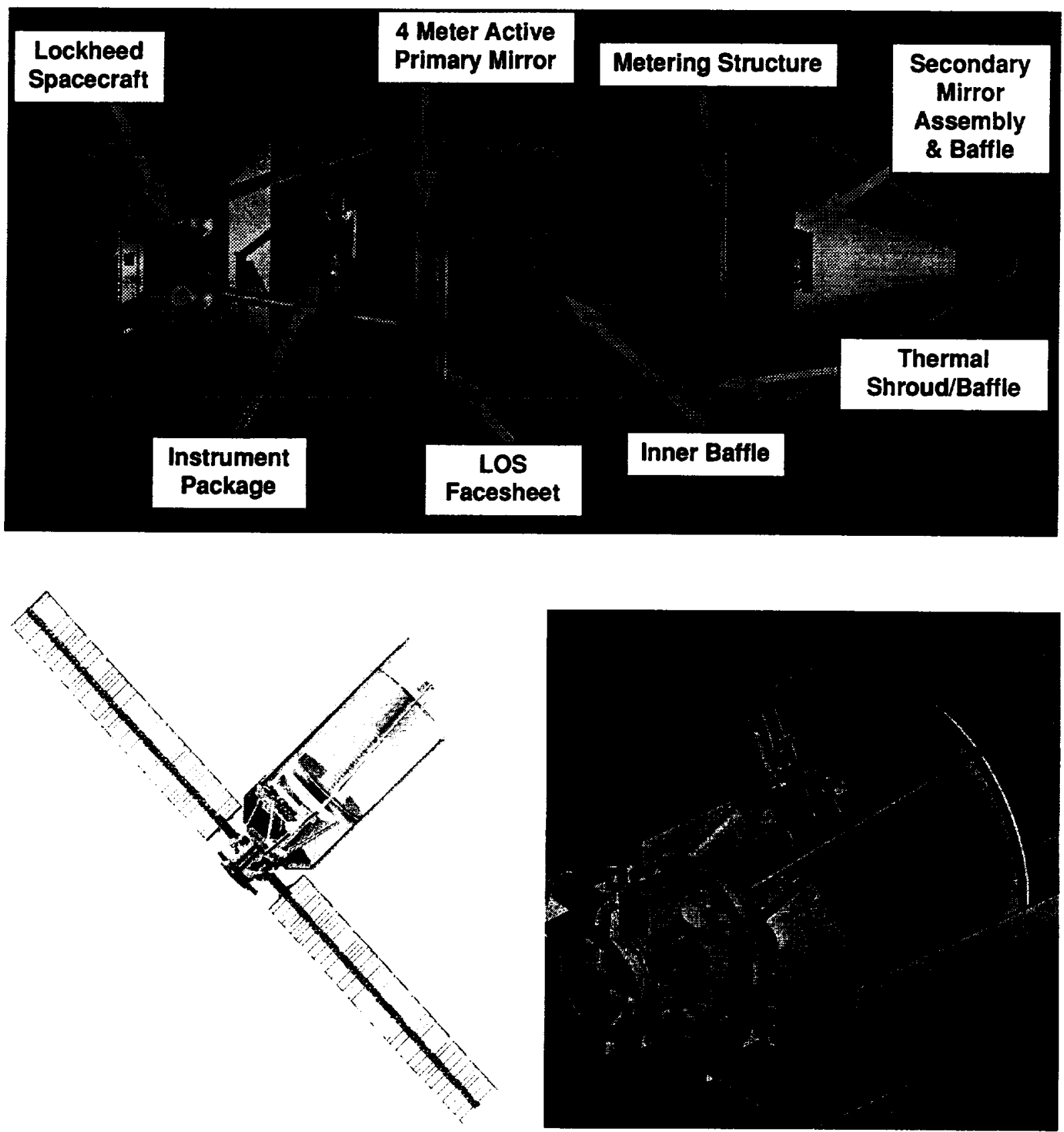

FIGURE 2.1 Key features of the ATD/NTOT. Components not used before in any astronomical telescope in space include a lightweight 4-meter mirror with active optics, an afocal design using a fast steering mirror and an on-board inertial reference for image stabilization, and a graphite polycyanate structure for the entire telescope assembly. Upper panel: This view of the ATD/NTOT packed inside the shroud of a Proton booster shows the layout of the principal components, including Lockheed's spacecraft bus - the F-SAT-and the thin, 4-meter primary mirror derived from Itek's Large Optical Segment (LOS) project. Lower left panel: A view of the ATD/NTOT in orbit with its solar panels extended. Lower right panel: A detail of the ATD/ NTOT showing (left) the F-SAT bus with its solar panels extended, (center) the instrument package, and (right) the rear face of the primary mirror and its adaptive-optics system. (Courtesy of Lockheed.) 


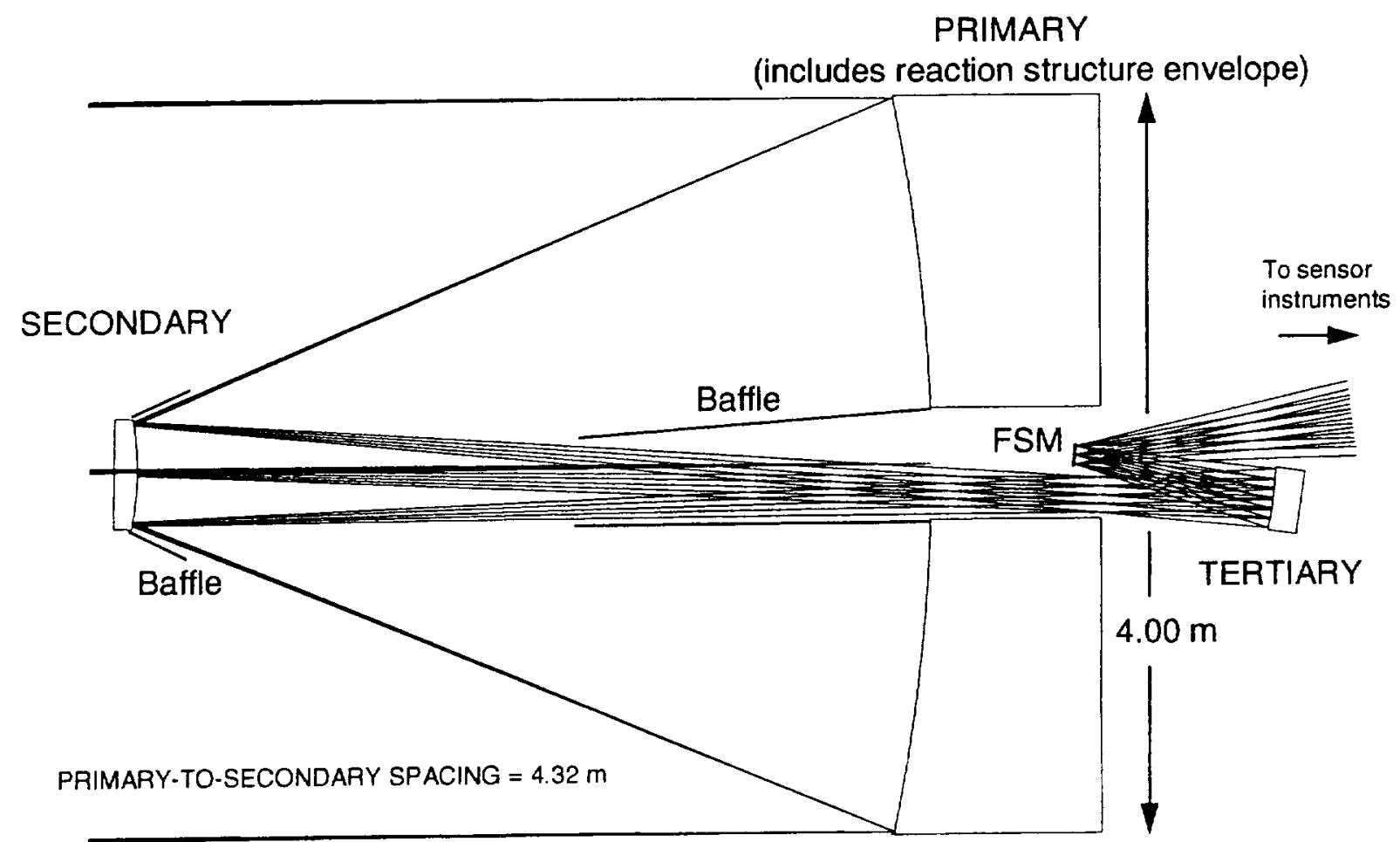

FIGURE 2.2 The ATD/NTOT's secondary and tertiary mirrors relay an image of the entrance pupil from the primary mirror to a fast steering mirror (FSM). The FSM is followed by a dichroic beam splitter that divides the light into separate infrared and visible beams at a wavelength near 1 micron. These separate beams are subsequently directed to a number of different focal planes by additional beam dividers and reimaging optics. The visible branch contains a wavefront sensor (not shown) used to assess the shape of the primary mirror and to generate correcting signals for the primary mirror's figure control actuators. (Courtesy of Lockheed.)

TABLE 2.1 Specifications of the ATD/NTOT's Primary, Secondary, and Tertiary Mirrors Based on Information Received from Lockheed and Itek

\begin{tabular}{|c|c|c|c|}
\hline Parameter & Primary & Secondary & Tertiary \\
\hline Aspheric type & $\begin{array}{l}\text { Paraboloid } \\
(\mathrm{CC}=-1.000)\end{array}$ & $\begin{array}{l}\text { Hyperboloid } \\
(\mathrm{CC}=-1.690)\end{array}$ & $\begin{array}{l}\text { Hyperboloid } \\
(\mathrm{CC}=-1.009)\end{array}$ \\
\hline \multicolumn{4}{|l|}{ Parent } \\
\hline Size & $400.0 \mathrm{~cm}$ & $58.9 \mathrm{~cm}$ & $38.6 \mathrm{~cm}$ \\
\hline F-Ratio & $\mathrm{f} / 1.25$ & $\mathrm{f} / 1.33$ & $f / 2.47$ \\
\hline Aspheric & $528.1 \mu \mathrm{m}$ & $101.3 \mu \mathrm{m}$ & \\
\hline Aspheric slope & $1.8 \mu \mathrm{m} / \mathrm{mm}$ & $2.8 \mu \mathrm{m} / \mathrm{mm}$ & \\
\hline \multicolumn{4}{|l|}{ Section } \\
\hline Size & & & $33.8 \mathrm{~cm}$ \\
\hline Offset & & & $2.527 \mathrm{~cm}$ \\
\hline F-Ratio & & & $\mathrm{f} / 2.82$ \\
\hline Aspheric & & & $8.15 \mu \mathrm{m}$ \\
\hline Aspheric slope & & & $0.3 \mu \mathrm{m} / \mathrm{mm}$ \\
\hline Footprint & $400.0 \mathrm{~cm}$ & $54.8 \mathrm{~cm}$ & $10.0 \mathrm{~cm}$ \\
\hline Test type & $\begin{array}{l}\text { Parabola test with } \\
\text { compensator }\end{array}$ & Hindle & $\begin{array}{l}\text { Parabola test with } \\
\text { compensator }\end{array}$ \\
\hline
\end{tabular}




\begin{tabular}{|ll|}
\hline \multicolumn{1}{|c|}{ Box 2.1 } & \multicolumn{1}{c|}{$\begin{array}{c}\text { Assumed Specifications of the ATD/NTOT Based on } \\
\text { Information Received from Lockheed and Itek }\end{array}$} \\
Primary mirror aperture & 4 meters \\
Optical configuration & 3 -mirror, afocal, anastigmat \\
Primary f-ratio & $1.25(1.7$ also possible) \\
Diftraction-limited field of view & 34 arc min \\
Afocal magnification & 40 (20 also possible) \\
Spectral band & 0.45 to $10 \mu \mathrm{m}$ \\
Static wavefront error of primary mirror & 0.07 micron \\
Obscuration (including support structure) & $15 \%$ \\
Coating & Silver \\
Operating temperature & $250 \mathrm{~K}$ (1000-km orbit) \\
& $\sim 200 \mathrm{~K}$ (Molniya orbit) \\
Line-of-sight stability & 0.0045 arc sec/axis in \\
& presence of spacecraft \\
& disturbances \\
On-board memory & 8 Gigabytes \\
& \\
\hline
\end{tabular}

The primary mirror's actuators correct figure errors below the Nyquist frequency of about 10 cycles per aperture. The resulting, post-correction figure error will then be the residual error below the Nyquist frequency and uncorrected errors at higher frequencies.

There are two principal sources for the residual wavefront error in the so-called baseline telescope design for the ATD/NTOT. First, on the basis of previous programs, Itek estimates that the primary mirror will have a surface accuracy of roughly $35 \mathrm{~nm} \mathrm{rms}$ and thus will contribute $70 \mathrm{~nm} \mathrm{rms}(2 \times 35)$ to the wavefront error. Second, the four additional optics will contribute an additional $37 \mathrm{~nm}$ to the telescope's wavefront error (based independently on test data supplied by Itek and by estimating errors individually for each optic based on size, speed, and conic). The combination of these two errors is $80 \mathrm{~nm}$ rms. The disproportionate contribution to this total due to the roughness of the primary mirror suggests a possible enhancement. Since obtaining improved surface accuracy at frequencies of 10 to 20 cycles per aperture is likely to be relatively inexpensive, it is possible that the final figure quality could be improved dramatically and cost-effectively by seeking a better figure on the primary mirror. Since scattered light scales with the square of the figure error, even modest improvements in figure can result in significant performance gains. These gains and their consequences are discussed in Box 2.3, "Enhanced Telescope Design."

\section{Point-Spread Functions}

To model the PSFs the task group generated a synthetic power spectrum of the errors in the surface figure to represent the figure error of the optics as filtered through the actuator system. A power-law spectrum was assumed and then filtered within 10 cycles per aperture. The integrated residual figure was normalized to that of the particular telescope design being evaluated (i.e., either the baseline or the enhanced design discussed in Box 2.3). The filtered and unfiltered power spectra are shown in Figure 2.3. Using this synthetic power spectrum of the primary, the assumed power spectrum for the other optical elements, and an assumed broadening due to jitter, PSFs were calculated at various wavelengths.

The PSFs and encircled energies of the baseline design in the visible and near-infrared regions are given in Figure 2.4. At a wavelength of 1 micron, the Strehl ratio is $\sim 0.75$. At 0.6 micron, the encircled energy in the central peak is $45 \%$ (this compares favorably with the prerefurbished HST's Strehl ratio of 0.15 ). The full width at half maximum (FWHM) at optical wavelengths is better than that for any current or planned facility. The 
Box 2.2 ATD/NTOT Weight Summary (in kilograms) Based on Information Received from Lockheed and Itek

\section{Primary Mirror Assembly}

Facesheet (17mm thick solid) $\quad 440$

$\begin{array}{ll}\text { Reaction structure } & 291\end{array}$

Actuators

$\begin{array}{ll}\text { Primary mirror assembly bipods } & 27\end{array}$

Central baffle $\quad 22$

$\begin{array}{lr}\text { Cabling } & 100\end{array}$

$\begin{array}{ll}\text { Subtotal } & 1,010\end{array}$

Secondary Mirror Assembly

Mirror (70\% lightweight)

Bipod flextures

Actuators

Reaction structure

Baffle

Subtotal

Tertiary Mirror Assembly

Mirror (50\% lightweight)

Bezel

Subtotal

Other

Metering structure

AFT metering structure

Instrument support structure

210

Shroud

Electronics

Fast steering mirror assembly

Wavefront sensor

Miscellaneous

Subtotal

168

41

73

9

35

37

DOD Instruments

680

Scientific Instruments

(up to) 900

F-SAT

1000

F-SAT Fuel

500

Contingency

TOTAL 


\section{Box 2.3 Enhanced Telescope Design}

As has been established, the roughness of the primary mirror dominates the system error in the baseline telescope design. Thus, improving it leads to a directly proportional improvement in the total system. This, in turn, allows much improved astronomical imaging and also allows a much better evaluation of the various components of the technology and how they contribute to the quality of the images. Many astronomical investigations push the limit of spatial resolution and require the maximum contrast in a scene. The extent to which this is needed for DOD's demonstration of technology is outside the task group's scope of expertise, although it seems likely that if the DOD missions involve reconnaissance rather than just target acquisition and tracking, then maximizing the contrast in the images may be important. It may also be important even for acquisition and tracking on faint targets.

ltek estimates that improving the surface of the primary mirror by a factor of two-to less than $20 \mathrm{~nm}$ rms compared to less than $40 \mathrm{~nm}$ rms as now specified--would cost approximately $\$ 100,000$. Assuming that this enhancement is made, ITEK's experience suggests that a requirement of $20 \mathrm{~nm}$ ms would lead to a primary mirror with an actual surface error of about $17 \mathrm{~nm}$ ms.

To assess this possibility, the task group analyzed a departure from the baseline in which the primary mirror and telescope-level residual wavefront errors are, respectively, 34 and $50 \mathrm{~nm}$ rms. It does not make sense to consider enhancing the primary's figure much (say, by more than another factor of two) beyond this level since, at this point, the system's figure error is being driven by the remaining optics in the telescope and focal plane.

The point-spread functions (PSFs) and encircled energies for the enhanced design at the visible and near-infrared wavelengths are given in Figure 2.5. The value of the enhanced figure requirements is evident from a comparison with Figure 2.4. The performance relative to the diffraction limit improves at longer wavelengths as shown in the two figures, although the full width at half maximum (FWHM) naturally increases in absolute units at longer wavelengths.

At infrared wavelengths, the system is approaching the diffraction limit in the baseline (Strehl $>0.8$ ), and so the gain with the improved primary is not large. At optical wavelengths, however, the image quality is dominated by errors in the primary mirror in the baseline, so that improving that single specification makes a large difference in both the Strehl ratio and the diameter of the circle with $50 \%$ encircled energy. (In the task group's crude modeling, the FWHM of the central peak is already very good and does not change significantly with the improvement in figure).

Even at 0.8 micron, an improved primary mirror ensures that the various sources of error are approximately comparable so that the various limitations on the telescope's performance can be reliably assessed at optical wavelengths where the performance is much more critical. At the shortest wavelengths, significant power from a point source is redistributed far from the central core so that the circle with $50 \%$ encircled energy is increasing as the wavelength decreases, even though the FWHM of the central peak is decreasing. The enhanced design pushes the wavelength of the minimum circle down to the vicinity of 0.6 micron.

Making this enhancement would lead to a dramatic improvement in the ATD/NTOT's astronomical performance at optical wavelengths. There should be an improvement by a factor of two in contrast between a point source and the background at a wavelength of 0.75 micron, leading to a corresponding improvement in the limiting magnitude of the system, since the dominant noise source at these wavelengths is the zodiacal light. The improvement in resolution is equally important for studying the spatial structure of sources at this range of angular sizes and could make the ATD/ NTOT dramatically better in spatial resolution than the Hubble Space Telescope, even with the planned Advanced Camera.

The ability to evaluate all aspects of the technology for their applicability to astronomy is also dramatically improved by the improved surface accuracy. There are many other sources of error that cause the PSF to deteriorate, including the stability of the field distortion, the stability of the tracking, and so on. When the contribution of these sources is small compared to the errors of the primary mirror surface, it becomes much more difficult to evaluate the degree to which these sources are satisfactory. The task group concludes, therefore, that this enhancement is very desirable and in subsequent discussions refers to the so-called enhanced telescope design frequently, comparing it with the baseline design where appropriate. 
relatively poor figure of the primary mirror, however, leads to a diameter for $50 \%$ encircled energy that is no better than that of the HST. The performance relative to the diffraction limit improves at longer wavelengths, although the FWHM naturally increases in absolute units at longer wavelengths. At infrared wavelengths $(\lambda>1 \mathrm{micron})$, the system is approaching the diffraction limit (Strehl ratio $>0.8$ ).

Not included in these calculations is any effect due to surface micro-roughness or to the $5 \%$ of the light striking the primary mirror that is diffracted wide of the image core by the "spider" supporting the secondary mirror. Itek has considerable experience in building mirrors with small micro-roughness, and published figures indicate a limit of $1 \mathrm{~nm}$ for the micro-roughness; the task group has not considered this issue in detail. The effect of the secondary supports is about five times larger for the ATD/NTOT than for telescopes with a more traditional truss design. Thus images with more greatly pronounced spider flares can be expected (Figure 2.6). The quantitative effects of the spiders can be approximated by multiplying the PSFs and encircled energies by 0.95 . Print-through of the actuators is included implicitly in the peak at 10 cycles of the task group's filtered surfacepower spectrum (see Figure 2.3), although circular symmetry is assumed and the print-through will enhance the effects at certain azimuths of the PSF.

The results of the task group's analysis are summarized in Table 2.2, which compares the baseline and enhanced (see Box 2.3) specifications for the primary mirror, taking into account the $5 \%$ loss due to diffraction on

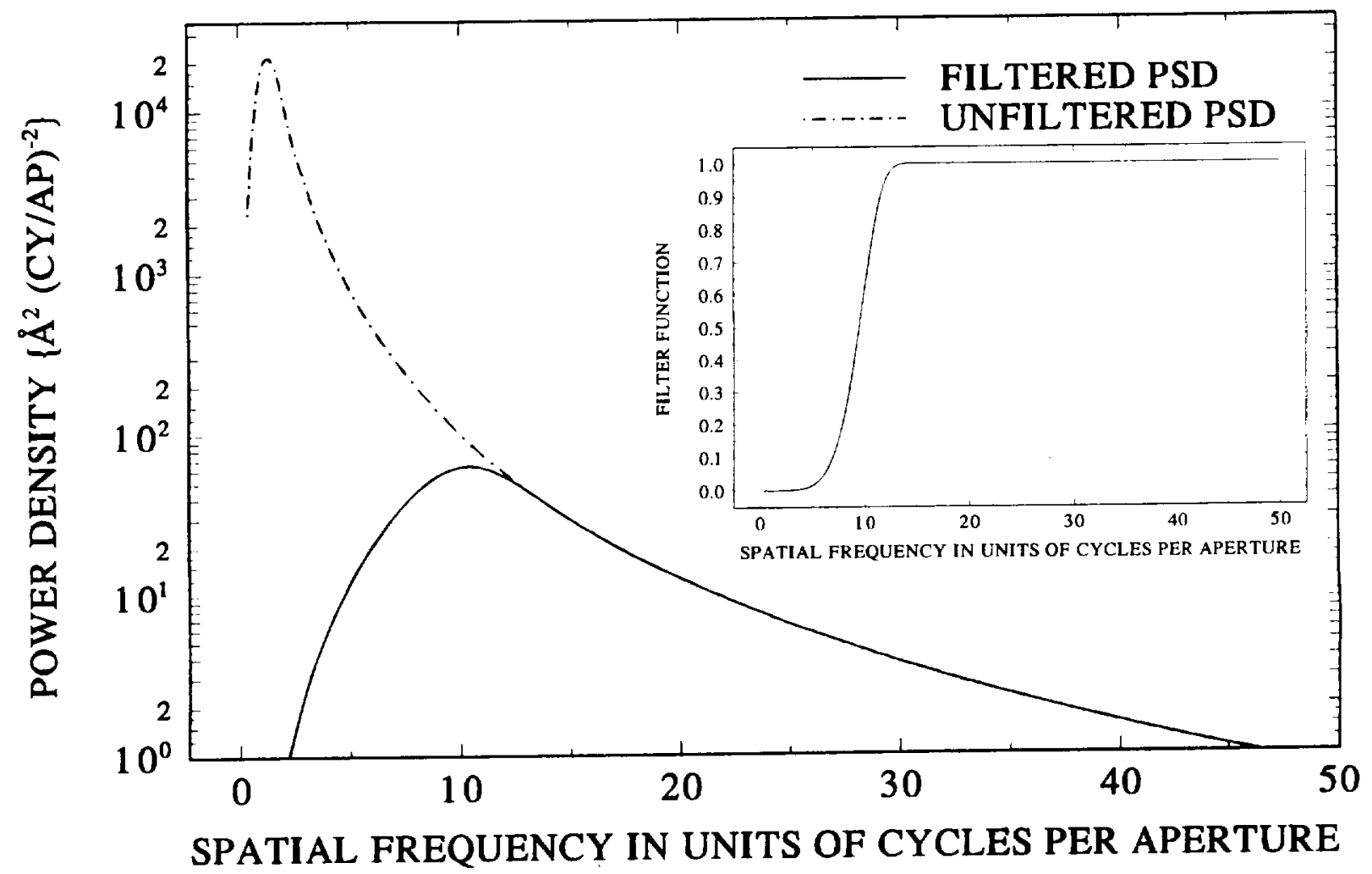

FIGURE 2.3 The synthetic power spectrum of surface figure errors generated by the task group to represent the figure error of the optics both before and after filtering through the actuator system. The filtered spectrum was found by assuming a power-law spectrum that was filtered to within 10 cycles per aperture using the inset filter function. 


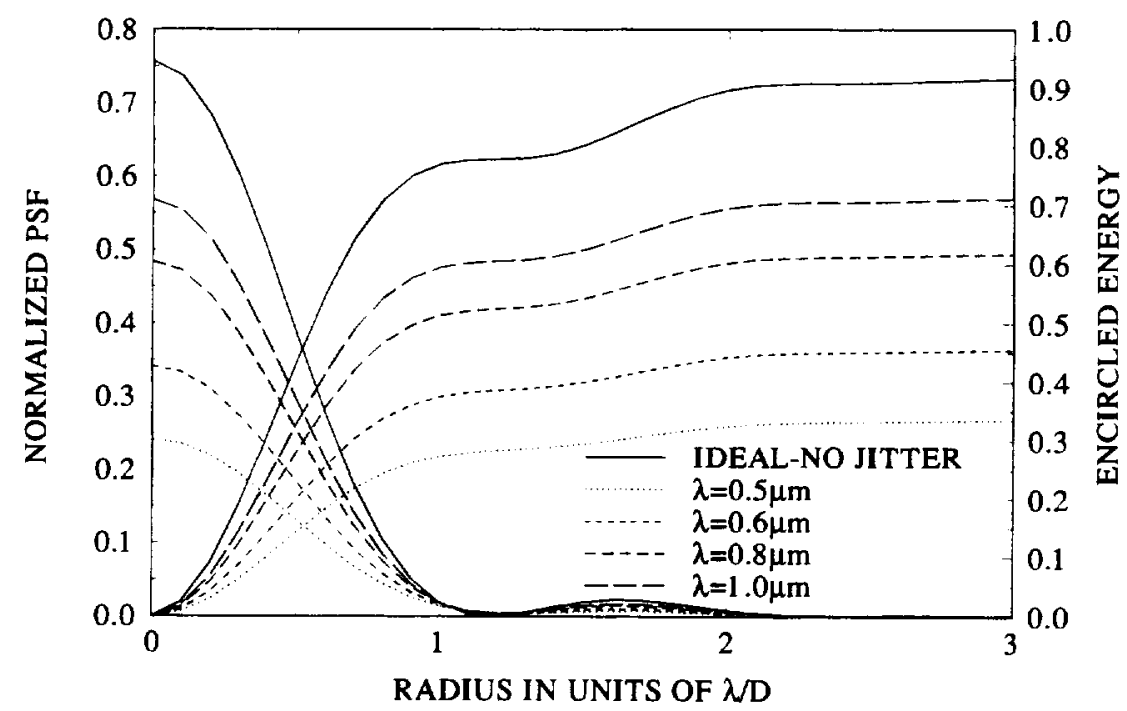

FIGURE 2.4 The task group's calculated point-spread functions (PSFs) and encircled energy curves are shown for the baseline design of the ATD/NTOT with a total wavefront error of $80 \mathrm{~nm}$ (i.e., for a primary mirror having a wavefront error of $70 \mathrm{~nm}$ and assuming a pointing jitter of 6 milliarc sec). All PSFs are normalized such that their integral over all space is unity when focal-plane coordinates are expressed in units of $N D$ (where $D$ is the telescope's aperture). By scaling out the wavelength in this manner, PSFs corresponding to different wavelengths can all be compared to the same ideal curve.

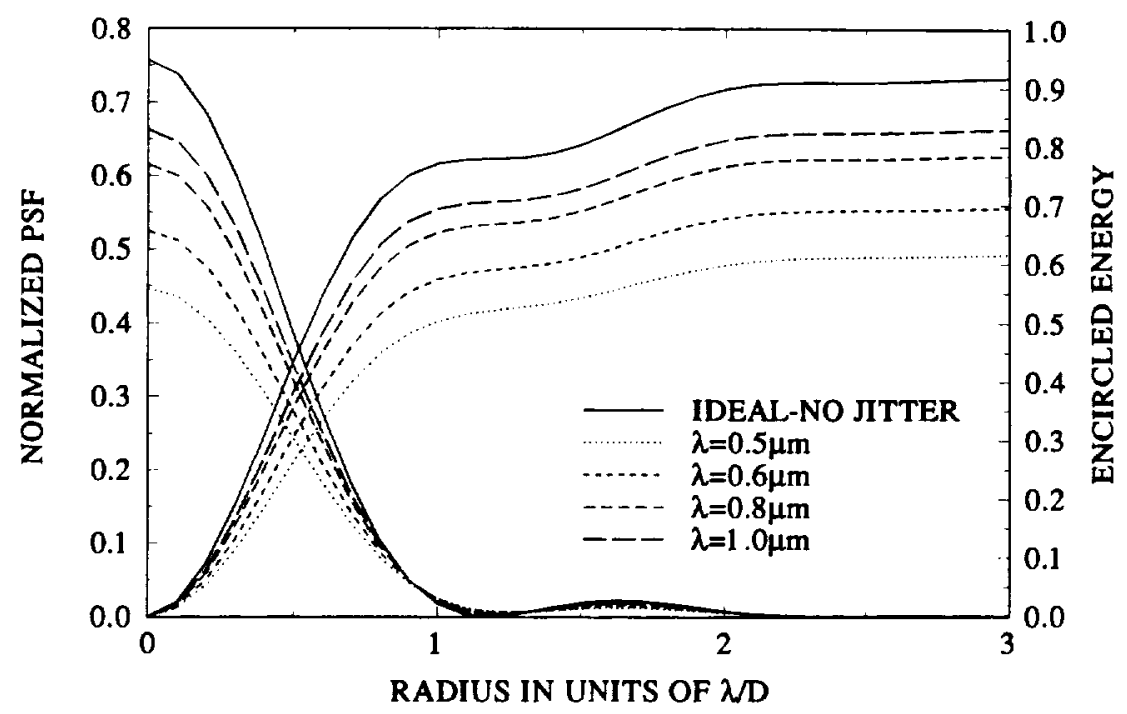

FIGURE 2.5 The task group's calculated point-spread functions (PSFs) and encircled energy curves are shown for an enhanced design of the ATD/NTOT (i.e., one with a primary mirror having a wavefront error of $35 \mathrm{~nm}$ and assuming a pointing jitter of 6 milliarc sec). As in Figure 2.4, all PSFs are normalized such that their integral over all space is unity. 

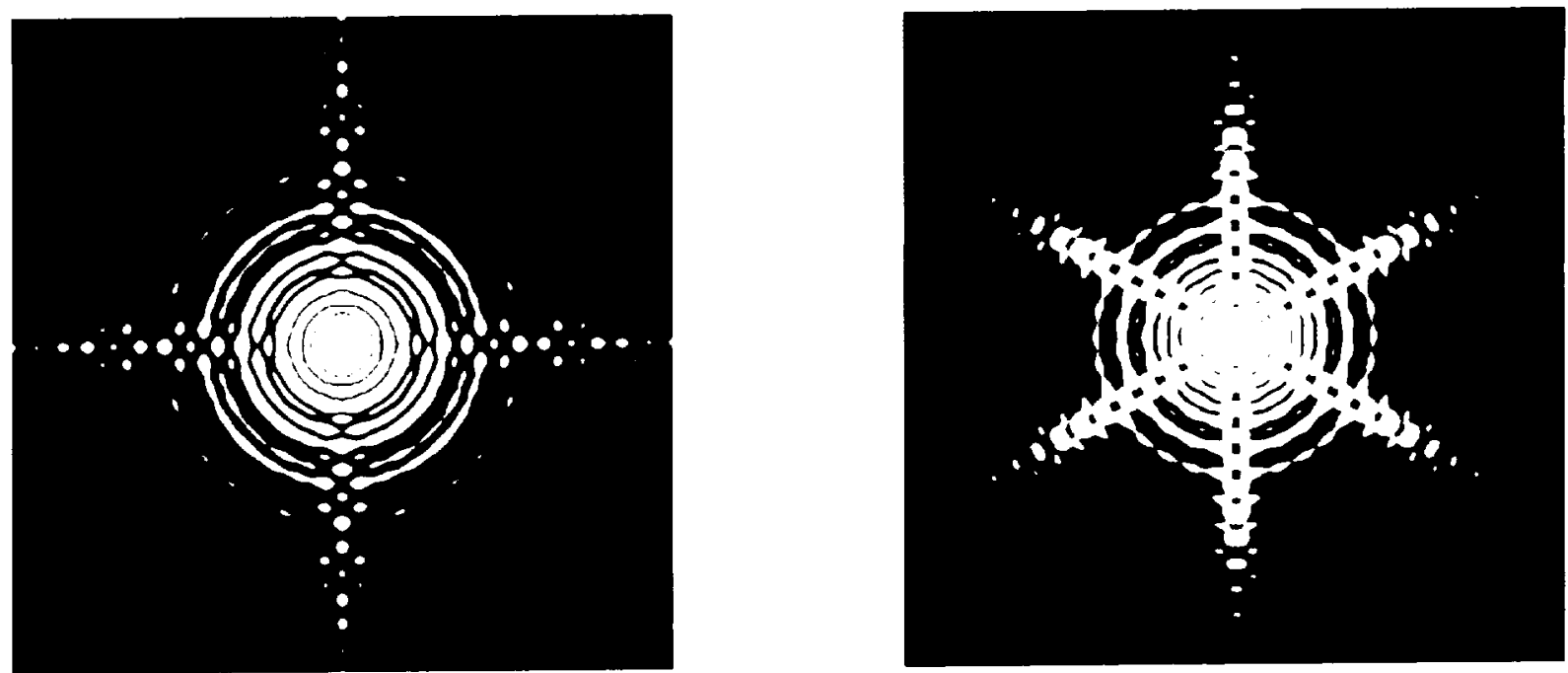

FIGURE 2.6 These synthetic images illustrate the task group's calculations comparing ideal diffraction-limited pointspread functions (PSFs) for the ATD/NTOT (right) and HST (left) optical systems. Both are normalized to unit intensity, and both are identically saturated at the center to bring up the spider flares. The spatial scale is in units of $\lambda D$ (where $D$ is the aperture). These images are intended to demonstrate the differences in the basic structure of the two PSFs due to the different means used to support each telescope's secondary mirror. Since the two telescopes have different diameters and Strehl ratios, these figures would have to be rescaled in order to represent absolute energy content. At a wavelength of 0.6 micron, the Strehl ratio of the HST's optical system is about twice that of the baseline ATD/NTOT, but the latter has 2.8 times the collecting area and its PSF is 1.7 times narrower at the same wavelength.

the truss, the inaccuracies of the other mirrors, and the jitter in the pointing of the ATD/NTOT. Table 2.2 also includes comparative data for the HST and also the Keck telescope (both with and without adaptive optics). It is clear that the enhanced design, which uses off-the-shelf technology, offers significant advances in astronomical performance over that of the HST, even taking into account the Advanced Camera that is currently under development for use with the HST. While not directly comparable, because of the effects of Earth's atmosphere, the enhanced ATD/NTOT has a near-infrared performance rivaling that expected from the Keck telescope when it is eventually outfitted with adaptive optics.

\section{FOCAL-PLANE SUITE}

The baseline suite of focal-plane instruments is defined by the needs of nonastronomical users of the ATD/ NTOT telescope. These instruments are summarized in Table 2.3. The beam from the FSM passes through a dichroic beam splitter that yields spatially separated infrared and optical beams. These in turn pass through neutral beam splitters and imaging optics (Figure 2.7).

The infrared detector of primary astronomical interest is the indium antimonide (InSb) array, assumed to be one of the new $1024 \times 1024$ arrays soon to be available from Hughes Santa Barbara Research Center. When operated at appropriately cold temperatures, this detector has state-of-the-art astronomical capabilities. The baseline design includes a pixel size of 0.05 arc sec. The task group assumes that the final scale of the imaging optics will be easily adjustable during the detailed design phase if it is decided to optimize the sampling of the PSF. It also assumes that the array will have the capability of subarray readout and/or many-amplifier fast readout that would allow, for example, multiple-sample reading of the array during a long integration.

The passive, visible, fine-tracking arrays are appropriate for some astronomical applications. But in the baseline design, they are line-transfer arrays with alternate rows masked for readout. Moreover, the amplifiers on the chips are not of the low-noise, low-speed design needed to minimize the readout noise. These arrays have 
TABLE 2.2 Summary of Optical Performance for ATD/NTOT, Keck, and HST

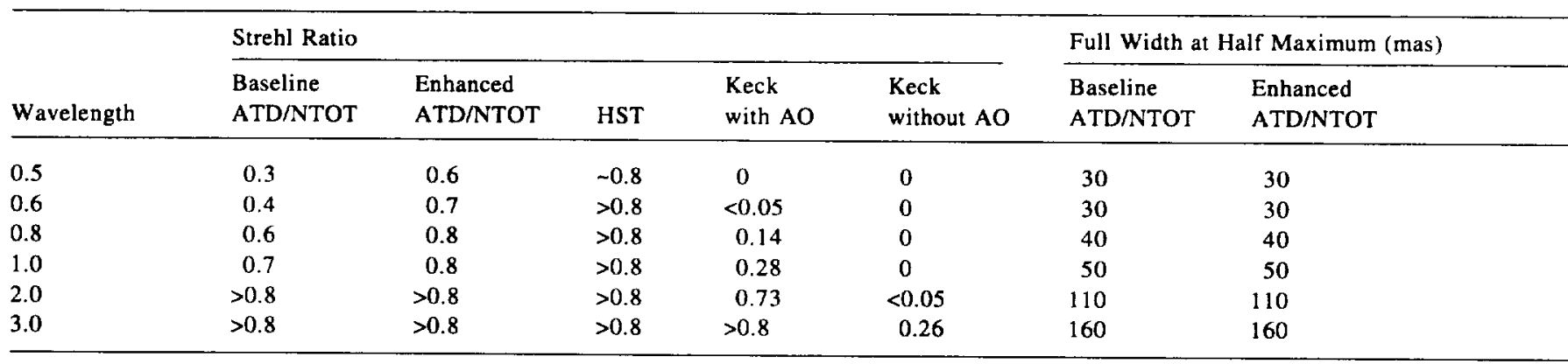

NOTE: The values for the HST correspond to what would be delivered by the optical telescope assembly, corrective optics, and a wellsampled detector (such as the Faint Object Camera at visible wavelengths). The values for the Keck telescope assume that the telescope and its adaptive-optics ( $\mathrm{AO}$ ) system are behaving properly and that the atmosphere is in the 25 th percentile (better than 0.35 arc sec). The image quality properties are dominated by the atmosphere for Keck without $A O$, and by the limited amount of correction that is applied using a single sodium laser artificial guide star. A Strehl ratio of 0.14 is comparable to that of the HST before the refurbishment mission.

0.031-arc-sec pixels, but because of the alternate-row masking they do not adequately sample the PSF. The task group understands from the briefings from Lockheed that the substitution of frame-transfer arrays for the linetransfer arrays may be consistent with DOD's technical goals. If so, this modification would go some of the way toward providing some, though not an optimum, optical astronomical capability.

The absence of a CCD of normal astronomical quality (i.e., one having a shutter, low dark current, slow readout, low-noise amplifiers, and so on) is a significant deficiency of the baseline focal plane. The baseline layout can easily accommodate an additional, large-format array that could be optimized for low noise. This possibility and other enhancements are discussed in Box 2.4, "Focal Plane Enhancements."

\section{ACQUISITION AND TRACKING}

Image stability at high and low frequencies is derived, respectively, from the IPSRU and from observation of guide stars with the fine-tracking sensors. The error signals from the two sources are combined such that the IPSRU dominates for frequencies above $10 \mathrm{~Hz}$ and the fine trackers dominate for frequencies below $1 \mathrm{~Hz}$. The fine trackers are read at $10 \mathrm{~Hz}$ and therefore contribute significantly to the tracking precision at frequencies up to about $3 \mathrm{~Hz}$. At these rates, the fine trackers can follow a star with $\mathrm{V}$ magnitude near 19.

TABLE 2.3 Baseline Suite of Focal-Plane Instruments

\begin{tabular}{|c|c|c|c|c|c|c|}
\hline Task & $\begin{array}{l}\text { Array } \\
\text { Type }\end{array}$ & $\begin{array}{l}\text { Field } \\
\text { of view } \\
(\operatorname{arc} \min )\end{array}$ & $\begin{array}{l}\text { Pixels } \\
\text { (no.) }\end{array}$ & $\begin{array}{l}\text { Pixel } \\
\text { Size } \\
(\operatorname{arc} s e c)\end{array}$ & $\begin{array}{l}\text { Aperture } \\
(\mathrm{cm})\end{array}$ & $\begin{array}{l}\text { Wavelength } \\
(\mu \mathrm{m})\end{array}$ \\
\hline Visible acquisition & Si CCD & $175 \times 175$ & $512 \times 512$ & 121 & 7.5 & $0.4-0.8$ \\
\hline Infrared acquisition & PtSi & $235 \times 175$ & $640 \times 480$ & 23 & 7.5 & $1.0-5.0$ \\
\hline Visible coarse tracking & Si CCD & $3.5 \times 3.5$ & $512 \times 512$ & 0.4 & 400 & $0.4-0.8$ \\
\hline Infrared coarse tracking & $\mathrm{PtSi}$ & $4.6 \times 3.4$ & $640 \times 480$ & 0.4 & 400 & 2.7 \\
\hline Active visible, fine tracking & $\begin{array}{l}\text { Si CCD (with } \\
\text { intensifier) }\end{array}$ & $0.5 \times 0.5$ & $512 \times 512$ & 0.06 & 400 & 0.53 \\
\hline Passive visible, fine track $(\times 2)$ & $\begin{array}{l}\text { Si CCD (line } \\
\text { transfer) }\end{array}$ & $1.1 \times 1.1$ & $2048 \times 2048$ & 0.03 & 400 & $0.4-0.8$ \\
\hline Infrared imaging & $\operatorname{InSb}$ & $0.9 \times 0.9$ & $1024 \times 1024$ & 0.05 & 400 & $0.9-5.5$ \\
\hline
\end{tabular}




\begin{tabular}{|c|c|c|c|c|c|c|c|}
\hline \multirow[b]{2}{*}{ HST } & \multirow[b]{2}{*}{$\begin{array}{l}\text { KECK } \\
\text { with AO }\end{array}$} & \multirow[b]{2}{*}{$\begin{array}{l}\text { KECK } \\
\text { without AO }\end{array}$} & \multicolumn{5}{|c|}{ Diameter of $50 \%$ Encircled Energy (mas) } \\
\hline & & & $\begin{array}{l}\text { Baseline } \\
\text { ATD/NTOT }\end{array}$ & $\begin{array}{l}\text { Enhanced } \\
\text { ATD/NTOT }\end{array}$ & HST & $\begin{array}{l}\text { KECK } \\
\text { with AO }\end{array}$ & $\begin{array}{l}\text { KECK } \\
\text { without AO }\end{array}$ \\
\hline 43 & 350 & 350 & $>160$ & 60 & $53 ?$ & 400 & 400 \\
\hline 50 & 340 & 340 & $>190$ & 50 & $65 ?$ & 390 & 390 \\
\hline 70 & 17 & 320 & 80 & 60 & $85 ?$ & 360 & 360 \\
\hline 85 & 21 & 300 & 80 & 70 & $105 ?$ & 350 & 350 \\
\hline 170 & 42 & 270 & 140 & 140 & $210 ?$ & 50 & 300 \\
\hline 250 & 62 & 62 & 200 & 200 & $320 ?$ & 70 & 280 \\
\hline
\end{tabular}

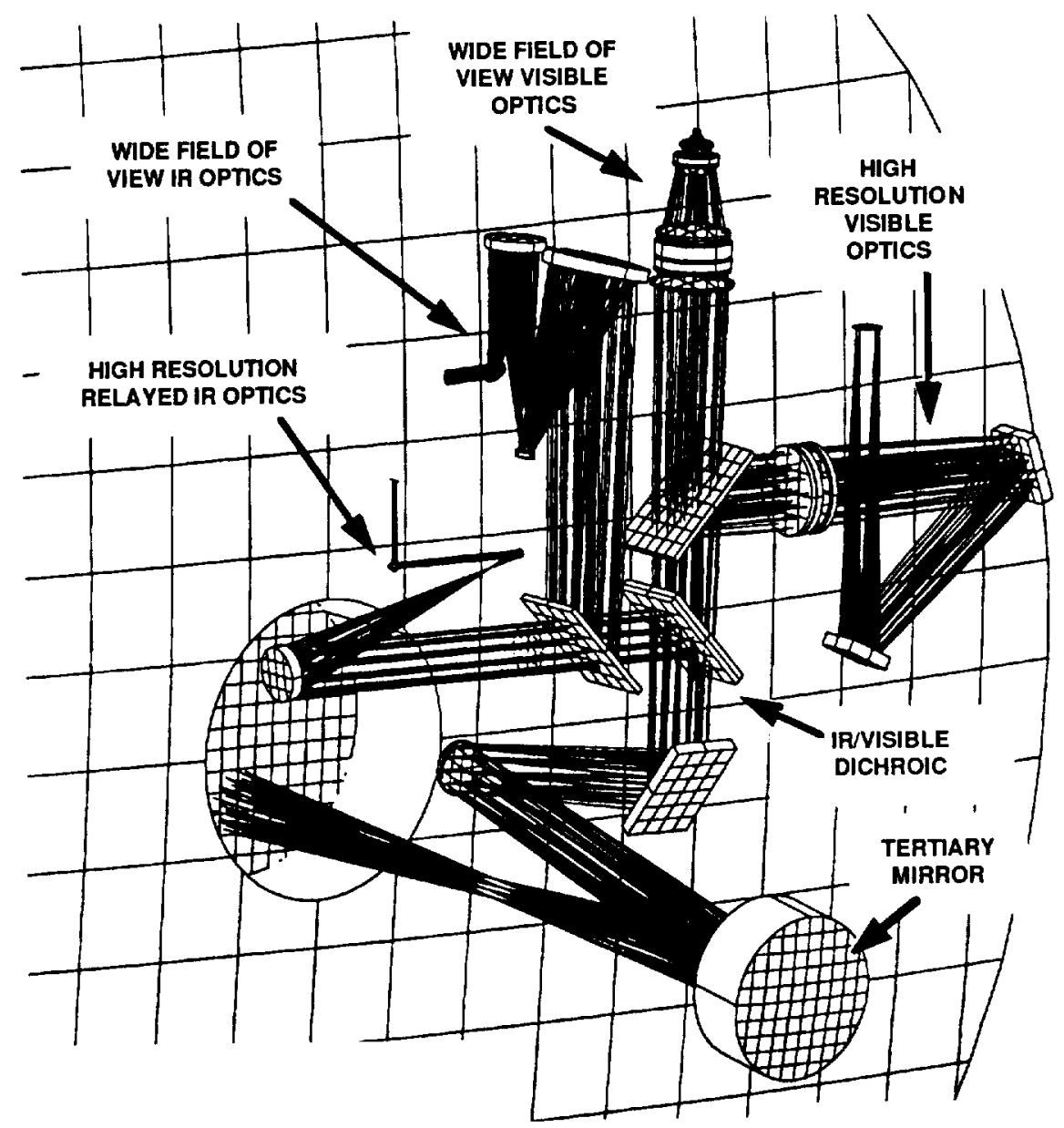

FIGURE 2.7 Schematic of a possible layout for the ATD/NTOT's focal plane. The beam from the fast steering mirror is reflected by a folding mirror and then passes through a dichroic beam splitter to spatially separate infrared and optical beams. These in turn pass through neutral beam splitters and reimaging optics to bring them to focal points on various array detectors (Courtesy of Lockheed.) 


\section{Box 2.4 Focal Plane Enhancements}

Hardware modifications to the spacecraft can be very expensive, particularly if they are driven by many scientific requirements. Nevertheless, some particular hardware enhancements seem desirable in light of potential deficiencies in the ATD/NTOT's baseline instrument suite.

\section{Optical Framing Camera}

The first and most important enhancement that should be considered is the addition of a suitable framing camera. This instrument should have the characteristics of an astronomical CCD camera. In other words, it should be capable of shuttered exposures, $100 \%$ sampling of the focal plane (no masked columns), and slow readout for minimal noise. A cheaper option would be to replace one or both of the line-transfer CCDs (i.e., ones in which every other column is masked for readout) in the fine-tracking detectors with frame-transfer CCDs. Other attributes to be considered include cooling for minimum dark current in long exposures and thermal control for photometric stability.

The effective area of the telescope is given by the ratio of the number of photons arriving at the focal plane per unit time from a given source to the number of photons incident per unit time per unit area on the aperture from the same source. In other words, it is the area of an aperture that collects as many photons as the real system would collect if it were $100 \%$ efficient. Thus the effective area takes into account the losses due to obscuration of the aperture by the secondary mirror, losses due to reflection at all surfaces, and the quantum efficiency of the detectors. The full area of a 4-meter circular aperture is $12.6 \mathrm{~m}^{2}$; the task group estimates that the tracking of CCDs will have an effective area of somewhat less than $3 \mathrm{~m}^{2}$ and believes that with careful attention to losses, an effective area near $6 \mathrm{~m}^{2}$ is possible with a high-quality framing $\mathrm{CCD}$.

A goal for this camera might be a readout noise of 3 electrons ms with a dark current of fewer than 10 electrons per hour per pixel. A thinned, back-illuminated chip is desirable to minimize the effects of cosmic-ray hits on the detector. Exposures should be precisely controllable from roughly 1 second to at least 10 minutes. Ultraviolet sensitivity is not needed, and the detector and camera should probably be optimized for sensitivity at 0.7 to 0.8 microns. The pixel size should fully sample the point-spread function (PSF) at the shortest wavelength at which the telescope will work, say at 0.5 micron, implying a pixel size near 25 milliarc sec. The size of the array should be as large as practically possible at the time of building the camera, certainly $2048 \times 2048$, and larger if mosaics of several chips are feasible. While a 2048 . chip is adequate for the technology demonstrations, the astronomical return from the mission is dramatically improved by increasing the size of the array as much as possible. The task group notes that there is ample space available for this camera in the focal plane as laid out in Lockheed's preliminary designs.

There are many groups in the country capable of designing and constructing such a camera. While it has made no careful estimates of the cost, the task group thinks that a suitable instrument would cost in the range of $\$ 3$ million to $\$ 15$ million. It notes that the far more sophisticated Hubble Advanced Camera for Exploration (HACE), recently selected by NASA, has a quoted price of $\$ 30$ million. The trade-offs should be explored between building the camera as part of the DOD sensor suite (perhaps even by the teams that built the cameras for Clementine), building it at a university as less than a class-A instrument, and building it to normal NASA specifications at a center such as the Jet Propulsion Laboratory or through a competitive bid. The important point is that the camera should be large but otherwise simple and that the procurement must be highly streamlined and cost-effective. However such a camera is built, the astronomical community must be involved at an early stage to ensure that the camera meets astronomical needs.

The justifications for adding an optical framing camera derive from both the technology demonstrations (see Chapter 4) and from subsequent astronomical operations (see Chapter 5). The alternate-column masking of the fine-tracking sensor in the DOD baseline focal plane prohibits the proper sampling of the PSF of images of point sources necessary to adequately characterize the performance of the telescope. Thus, adequate comparison of the images with the wavefront interferograms is impossible. Furthermore, the fast readout required for the fine-tracking sensors makes them unsuitable for the long exposures that are essential both for evaluating the tracking and guidance capability and for the astronomical observations. While the InSb array adequately samples the PSF at near-infrared wavelengths, the PSF at these wavelengths is significantly worse than that at shorter wavelengths because the diffraction limit is worse. The evaluation of the technology demands that images be properly sampled at the shortest possible wavelength, which in this case is near 0.5 micron. The camera thus provides the best end-to-end test of the integrated system, including all of the new technological components of the ATD/NTOT.

The inclusion of this camera is the enhancement that would most dramatically improve the astronomical retum from the mission. It would improve the quality of a Kuiper Disk search (see Chapter 5) by at least an order of magnitude. It is also the key factor that makes the search qualitatively different from one that could be done with the HST if the HST were devoted solely to that search for many months. The task group therefore views inclusion of the optical framing camera as the single most urgent addition of hardware to the focal plane. 


\section{Box 2.4 Continued}

\section{Cooled infrared Array}

Provided that the necessary funds are available, other enhancements to the baseline instrument suite should also be considered. The baseline InSb array is likely to be similar to the ones widely used in astronomy. However, the baseline design does not include maximum cooling to minimize the array's dark current, nor does it include special steps for passive cooling of the telescope structure and its optics. The InSb array will therefore be limited by local thermal emission, either of its own or from the telescope. Modifying the infrared design to optimize the cooling of the array and of the telescope structure is the second most important modification to the focal plane. The task group did not have sufficient details to evaluate this option critically but thinks it likely that a cooled infrared array would not be an expensive addition.

\section{Dedicated Focal Planes}

The imaging arrays in the baseline design have many optical surfaces between the detector and the telescope. This arrangement is particularly harmful in the infrared where the thermal emission from all these surfaces increases the background. It appears that it should be possible to pick off part of the telescope beam that is nearly on axis, namely the part that would strike the back of the fast steering mirror, and use it with suitable optics to feed a focal plane with a minimum of intermediate surfaces.

The task group has done no studies of the optics required nor determined how best to deal with tracking in this configuration, although using an internal steering mirror driven by the feedback signals from the fine-tracking array seems a possibility. While such an addition is likely to be expensive, a study of the costperformance trade-off seems justified before deciding arbitrarily whether such an enhancement is appropriate.

The IPSRU has demonstrated a stability of 7.2 milliarc sec rms, integrated over frequencies from 0.1 to 100 $\mathrm{Hz}$, with the spectrum of noise decreasing with increasing frequency. A briefing from IPSRU's manufacturer, Charles Stark Draper Laboratories, revealed that this performance was limited by the quality of the gyros in the demonstration unit. It is thought that higher-quality gyros could be obtainable and the noise reduced to $1.9 \mathrm{milliarc}$ sec rms.

The FSM can steer over a field of \pm 1.9 degrees, which translates to \pm 5.7 arc min on the sky after allowing for the 40:1 demagnification of the pupil (and the consequent 40:1 increase in the field divergence) and a factor-oftwo gain in the motion due to reflection. This mobility is adequate to eliminate the anticipated F-SAT jitter of 1.2 arc min (3-sigma excursion).

\section{OPERATIONS, ORBIT, COMMUNICATIONS, AND POWER REQUIREMENTS}

The baseline mission assumes that the ATD/NTOT is launched into a circular parking orbit with a $200-\mathrm{km}$ altitude using a Proton booster. As of early 1995 this launcher had a 19-year record of success (some 190 launches), averaging about 10 launches per year and with a success rate (last 50 moving average) of $96 \%$. It is not clear at this time whether DOD's mission would be carried out from a low circular orbit, such as the parking orbit, or from an elliptical orbit that carries the spacecraft to much higher altitudes. This choice has direct implications for the way in which astronomical demonstrations are conducted as well as for any longer-term astronomical research programs. However, either choice can be accommodated, since the baseline mission includes an eventual boost to a high-apogee, elliptical orbit.

The ATD/NTOT mission has a design lifetime of 1 year, and Lockheed's experience shows that such a mission, once past initial failures, has a high probability of lasting at least 5 years. The DOD mission is anticipated to take 6 to 12 months to complete. Since the demonstrations of astronomical capability must, at least in part, be carried out after the DOD mission, the task group has assumed that there will be an astronomical phase to the mission. For purposes of further discussion, 1 year is assumed for the national security mission and 5 years for the astronomical mission. 
The standard Proton booster (the D-1-e model) has four stages, with the fourth stage normally being used to inject the payload into its final orbit. The fourth-stage shroud, however, is not large enough to accommodate a 4meter telescope. Thus, the baseline ATD/NTOT mission assumes the use of the three-stage variant of the Proton (the D-1 model) and additional propulsion modules. These stages will boost the spacecraft into a $200-\mathrm{km}$ parking orbit that, because of launch-azimuth restrictions at Baikonur Cosmodrome, is limited to an inclination of 51.6, 64.8 , or 72.7 degrees. ${ }^{1}$ The baseline mission briefed to the task group would use the additional propulsion modules to boost the spacecraft at some time, either shortly after launch or after completing demonstrations of defense technology, from the parking orbit to a Molniya orbit (named after the class of Russian communications satellites that use this orbit). This type of orbit has an inclination of 63.4 degrees, is very eccentric, and has a nominal period of 12 hours. Perigee occurs at high southern latitudes at an altitude of about $400 \mathrm{~km}$, while apogee is at a correspondingly high northern latitude at a nominal altitude of $22,000 \mathrm{~km}$. Although some of the demonstrations of astronomical technology can readily be carried out from the low-altitude, circular, parking orbit, the Molniya orbit is the one in which most demonstrations of astronomical technology and essentially all astronomical observations for scientific purposes would be carried out. The use of alternative launch vehicles and possible orbital enhancements are discussed in Boxes 2.5 and 2.6, respectively.

In the baseline mission, the ATD/NTOT spacecraft carries some $500 \mathrm{~kg}$ of hydrazine for attitude control and maneuvering. It is expected that less than $10 \%$ of this fuel would be used in DOD's technology demonstrations, leaving significant maneuvering capability for a long-duration mission. In fact, the fuel reserve is more than enough for months of astronomical technology demonstrations and 5 years of astronomical observations.

The baseline communications capability is 5 megabits per second averaged over each orbit, and on-board data storage is 8 gigabytes. Use of the worldwide Air Force Satellite Control Network for mission control and data readout is assumed. The transmitter-antenna combination assumed for the baseline mission has an 8-dB margin above the minimum, at the apogee of the Molniya orbit, for a $10^{-6}$ bit error rate. This communications capability is sufficient to reduce the bit error rate significantly. The task group assumes that the spacecraft will have a

\section{Box 2.5 Alternative Launch Vehicles}

The selection of the launch system is critical to maintaining an overall affordable cost envelope. The basic mission mass to be put into low Earth orbit is of the order of $3900 \mathrm{~kg}$, including the F-SAT bus but with no additional scientific instruments or weight contingency. In addition, the current design requires a shroud diameter slightly greater than 4 meters, which must couple physically and dynamically to the selected launch vehicle. In the past, only U.S. launch vehicles would have been considered, including the Shuttle, Titan IV, and Atlas II, all at a cost considerably in excess of $\$ 100$ million. A broader list would include the European Ariane 4 and 5 (also very expensive) and the Chinese (PRC) Long March. With the end of both the Cold War and CO-COM trade restrictions with former Soviet republics, Russia's large, reliable, and relatively inexpensive space boosters are becoming increasingly available for consideration. A preliminary analysis indicates that the Molniya-A, Zenit, and Proton boosters would be capable of lifting the F-SAT bus and the ADT/NTOT to low Earth orbit. The Zenit and Proton are of particular interest because each has a U.S. partner (Boeing and Lockheed-Martin, respectively), and the Proton, which will be used as the supply vehicle for the International Space Station Alpha, has a new shroud with 4.2-meter internal diameter. With the Proton's low Earth orbit lift capacity of some $22,000 \mathrm{~kg}$, one or more additional large satellites could be included to share the estimated $\$ 60$ million cost. The Molniya-A is another newly available and highly successful (over 1000 launched to date) launcher that has an upperstage integral with a 4.1-meter shroud and that can lift $7000 \mathrm{~kg}$ into low Earth orbit and more than $2700 \mathrm{~kg}$ directly into the desired Molniya orbit, for an estimated price of about \$15 million.

This summary indicates that numerous boosters are suitable for placing the ADT/NTOT into orbit and that Russian boosters are very attractive from the standpoint of price, availability, reliability, and existing shroud availability. Further, the availability of existing Russian upper stages and the ability of Russian launchers to lift excess weight could lead to further cost reductions in the basic telescope system itself, for an overall mission cost lower than that originally projected by Lockheed and Itek. The main issue not addressed so far is one of policy, that of DOD purchasing a Russian launch system. The benefits suggest strongly that this approach should be seriously considered. 


\section{Box 2.6 Special Orbits}

The infrared performance of the ATD/NTOT is likely to be maximized if the telescope is operated at as low a temperature as possible. The largest obvious contributors to the heating of the telescope are sunshine (the same for al geocentric orbits) and earthshine (varying drastically with geocentric distance and direction). Studies of alternative orbits, from the point of view of both cooling and efficient operations, should be carried out during the final design phase. The orbits to be considered might include IRAS-like polar orbits orthogonal to the solar direction, IUE-like geosynchronous or even geostationary orbits, and SIRTF-like heliocentric orbits. The current communications capability might be inadequate for some of these (it would certainly be inadequate for the heliocentric orbit), and some orbits may be incompatible with DOD's need for evaluating its technology. These considerations deserve further exploration.

capability for lossless data compression, by at least a factor of two for essentially all data and by a factor of five for many types of data. It also assumes that some on-board processing will also be available that can, for example, take the median of several images to remove cosmic-ray hits (in cases for which that is desired) so that only median images need be transmitted to Earth. These assumptions provide a wide margin for the anticipated load of communications during the astronomical phases of the mission.

The power supplied by the F-SAT bus in the baseline mission is estimated at more than $3 \mathrm{~kW}$ at the end of a 6-year lifetime. Of this, approximately $1200 \mathrm{~W}$ would be used for spacecraft operations and communications, leaving some $2 \mathrm{~kW}$ for payload components. It is assumed that the payload provided for the DOD's technology demonstrations will take only a small fraction of this capability. Thus at least $1 \mathrm{~kW}$ of power should be available at the end of the ATD/NTOT's anticipated lifetime for any additions to the payload required by the astronomical tasks.

\section{REFERENCE}

1. Proton Launch Vehicle and Launch Services: User's Guide, Lockheed-Khrunichev-Energia International, Inc., San Jose, Calif., 1993. 


\section{Scientific and Operational Niches}

What is the most appropriate astronomical use for a technology demonstration such as the ATD/NTOT? Clearly a variety of technical capabilities must be demonstrated, and they are discussed in Chapter 4 . The task group also foresees a high probability that the telescope will have the capability to carry out important astronomical research. Indeed, several projects that seem to be particularly worthy are discussed in Chapter 5 .

But to choose which demonstrations and research programs to emphasize, it is critical to understand those areas in which the ATD/NTOT might have significant advantages over those other facilities likely to be in operation at the end of this decade. To this end, the task group considers two aspects of the telescope: its performance at near-infrared and optical wavelengths, and its potential operational modes.

\section{NEAR-INFRARED AND OPTICAL PERFORMANCE}

Earth's atmosphere hampers infrared observations from terrestrial observatories in several ways:

- Broad regions of the spectrum are blocked by strong absorption from $\mathrm{H}_{2} \mathrm{O}$ in the atmosphere;

- These absorption bands emit thermal radiation characterized by the mean temperature of the troposphere;

- Strong emission by $\mathrm{OH}$ makes the sky very bright at wavelengths from about 0.7 to 2 microns; and

- Turbulence, primarily in the tropopause, degrades a telescope's theoretical spatial resolution by introducing rapidly varying phase errors on scales smaller than the size of the aperture of the telescope.

Moreover, a telescope's own support structure and mirrors, which ideally are in thermal equilibrium with their surroundings, emit thermal radiation characterized by the emissivity of the mirrors and the ambient temperature $(\sim 280 \mathrm{~K})$.

The top panel in Figure 3.1 shows a theoretical model of the atmosphere's absorption-line spectrum, at wavelengths between 2 and 10 microns, above the 13,800-foot summit of Mauna Kea-one of the world's best sites for infrared astronomy. As the figure shows, the spectral range from approximately 2.4 to 3.4 microns is blocked. As the task group argues here and in Chapter 5, this inaccessible region is especially important for cosmological observations.

The bottom panel in Figure 3.1 shows the background radiation that will be seen by the infrared-optimized, 8 meter Gemini telescope at Mauna Kea. The two most important components of this background are the thermal emission from the mirrors (assumed to have a low emissivity of 0.03 ) and the thermal emission and airglow from 
the atmosphere. The radiation background seen by Gemini is orders of magnitude brighter than the limiting background due to thermal emission and scattered light from zodiacal dust (the lowermost curve in the bottom panel of Figure 3.1).

Infrared observations from space are limited only by a telescope's collecting area, thermal emission, and the natural background set by zodiacal dust. Figure 3.2 shows the zodiacal background at an ecliptic latitude of 45 degrees, calculated using a standard set of assumptions. ${ }^{1}$ At wavelengths shorter than the minimum at approximately 3.8 microns, the background rises as scattering by the zodiacal dust becomes more efficient. At wavelengths longer than the minimum, the background rises due to thermal emission from optically thin zodiacal dust.

A primary goal of space infrared astronomy is to reduce the thermal emission from the telescope below the background. This reduction is readily achievable at wavelengths between 2 and 3 microns using passive cooling techniques. The natural background in this spectral region is more than five times darker than the darkest part of the optical spectrum near 0.5 micron.

Figure 3.2 shows the thermal background from the ATD/NTOT for two different operating temperatures: the baseline value of $200 \mathrm{~K}$, and a scientifically more desirable temperature of $160 \mathrm{~K}$ (see Box 3.1, "Enhancing Infrared Performance"). The figure includes the background for the 8-meter, infrared-optimized Gemini telescope for comparison. If the ATD/NTOT operates at a temperature of $200 \mathrm{~K}$, it will be background limited and therefore able to make the deepest infrared images, in the wavelength range from 2.0 to 2.4 microns, while still having substantially lower background at longer wavelengths than any other telescope.

The ATD/NTOT will be background limited in the 2.0- to 2.4-micron band only if its baseline InSb detector
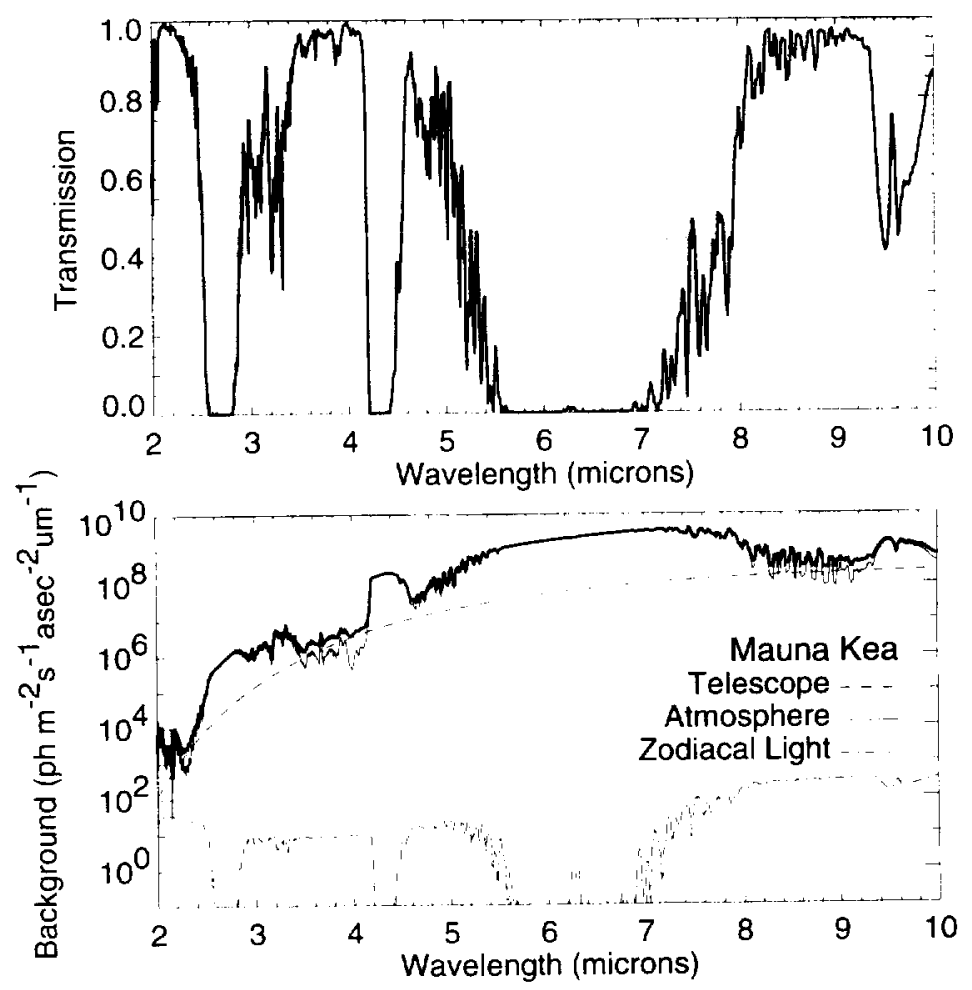

FIGURE 3.1 Upper panel: A theoretical model of the absorption-line spectrum of Earth's atmosphere, in the spectral region 2 to 10 microns, above Mauna Kea. Lower panel: Sources of the background radiation in the 2- to 10-micron region that will be seen by the infrared-optimized, 8-meter Gemini telescope on Mauna Kea. 


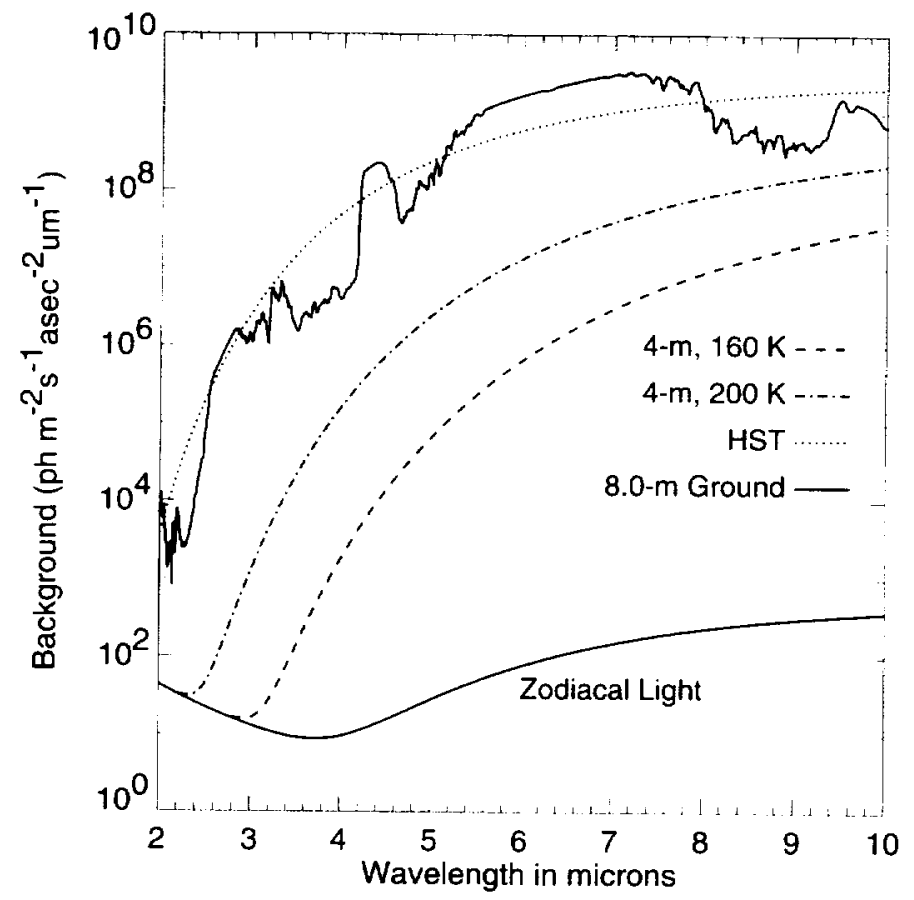

FIGURE 3.2 The zodiacal background at an ecliptic latitude of 45 degrees is compared with the thermal emission from the ATD/NTOT (assuming the effective emissivity from the 10 mirrors in the optical path to the $\mathrm{InSb}$ detector is 0.20 ) when operating at $200 \mathrm{~K}$ (baseline) and also at a more desirable value of $160 \mathrm{~K}$ (enhanced). Note that the ATD/ NTOT is background limited at wavelengths up to 2.4 and 3.0 microns for the baseline and enhanced temperatures, respectively. Thermal emission from the Hubble Space Telescope and the ground-based, 8-meter, infrared-optimized Gemini telescope is included for comparison.

has sufficiently low dark current and read noise. The task group calculated the noise requirements by assuming that the bandpass of a filter at 2.2 microns is 0.4 microns and that half the 2.2 -micron photons incident on the aperture are detected by the InSb array. The zodiacal background rate is then $\sim 0.2$ electrons per second per 50 milliarc-sec pixel. To be background limited, the dark current in the array must be lower than $\sim 0.2$ electrons per second per pixel. Assuming a 1000-second integration, the background shot noise will be $\sim 14$ electrons per pixel. Consequently, the rms read noise per pixel must be lower than this value to realize background-limited exposures.

\section{Box 3.1 Enhancing Infrared Performance}

There is considerable advantage in lowering the temperature of the telescope from the baseline value of $200 \mathrm{~K}$ to 160 $\mathrm{K}$. As Figure 3.2 indicates, the telescope is then background limited to a wavelength of 3 microns where the zodiacal background is twice as dark as at 2.4 microns. Cooling the telescope would, as indicated in Figure 3.3, result in a limiting magnitude approximately 2 magnitudes fainter over the interval from 3 to 8 microns than can be achieved in the baseline. If the telescope flies with low-noise detectors that operate at these wavelengths, every effort should be made to reach the goal of $160 \mathrm{~K}$. 


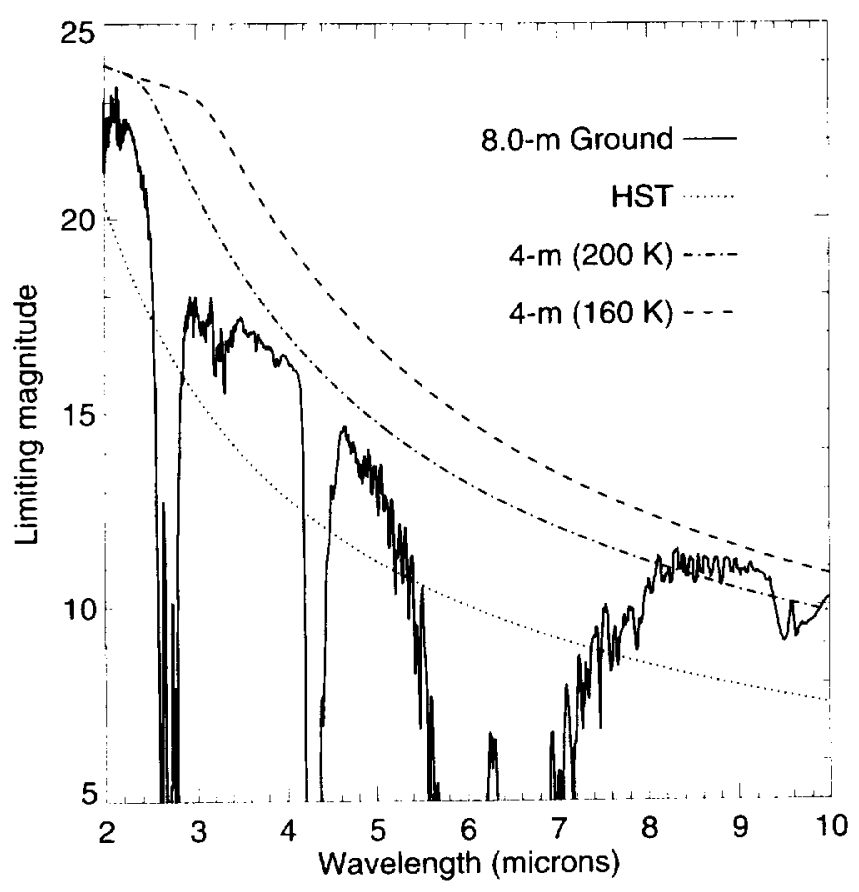

FIGURE 3.3 The limiting magnitudes of both the baseline (at $200 \mathrm{~K}$ ) and an enhanced (at $160 \mathrm{~K}$ ) ATD/NTOT are compared with those of the Hubble Space Telescope and the 8-meter, infraredoptimized Gemini telescope. Note the ATD/NTOT's advantage over the much larger Gemini telescope in the region between 2 and 3 microns. Also note that the performance of Gemini in this atmospheric window reflects the goal of $3 \%$ emissivity for that tele scope.

Figure 3.3 shows the limiting magnitude for the ATD/NTOT and the Gemini telescope at wavelengths between 2 and 10 microns. The calculation assumes that the former is diffraction limited at all wavelengths and that the latter instrument uses adaptive optics to achieve a near-diffraction-limited image with a Strehl ratio of 0.5 .

At a temperature of $200 \mathrm{~K}$, the 4-meter ATD/NTOT has a fainter limiting magnitude at all wavelengths between 2 and 8 microns than does the 8-meter Gemini telescope. In the limited spectral windows transparent from the summit of Mauna Kea, the ATD/NTOT will see objects at least 1 magnitude fainter than the Gemini telescope. The smaller aperture wins because of the lower operating temperature.

The biggest advantage of the 4-meter telescope is its deep limiting magnitude at all wavelengths between 2 and 3 microns, where the Gemini telescope is severely limited by the strong telluric absorption centered at 2.7 microns. Because the 5- to 8-micron interval is strongly blocked from the ground, exploiting the ATD/NTOT's performance in this region would be especially valuable, provided a suitable infrared detector was added to its instrument suite.

As described in Chapter 2, the baseline optical system yields images with a full width at half maximum (FWHM) that is considerably smaller than the FWHM of images obtained with the HST or with any other currently existing or planned facility. However, the optical quality of the primary mirror is low, a characteristic that leads to the rather poor value for the Strehl ratio and for the diameter encircling 50\% of the total energy.

Many problems requiring high spatial resolution can be carried out with the baseline system. However, the limits on the image quality are dominated by the surface accuracy of the primary mirror, making it difficult to evaluate other contributors to the error budget for image quality. Furthermore, the small fraction of the energy in 


\section{Box 3.2 Enhancing Optical Performance}

With an enhanced primary mirror, the ATD/NTOT has an rms wavefront error of $\sim \lambda / 10$ at 0.5 micron. This corresponds to a Strehl ratio of $\sim 0.6$ and gives a point-spread function (PSF) with a full width at half maximum (FWHM) of $\sim 30$ milliarc sec (mas). Objects at cosmological distances are better observed at longer wavelengths because of redshift and $\mathrm{K}$-corrections, and the zodiacal background is decreasing toward its minimum at $\sim 3$ microns (see Figure 3.3). Furthermore, the distant primitive bodies of the solar system, such as those in the Kuiper Disk, are best surveyed in the far red, where they are brighter than in the visible. The task group assumes, therefore, that the CCD in the optical framing camera (see Box 2.4, "Focal Plan Enhancements," in Chapter 2) will be optimized for a wavelength of $\sim 0.8$ microns. At this wavelength the Strehl ratio will be $\sim 0.8$ (i.e., the telescope is nearly diffraction limited) and the PSF's FWHM will be $\sim 40$ mas. Using these numbers and assuming that each of the silver-coated mirrors has $98 \%$ reflectivity, the task group estimates that $52 \%$ of the photons incident on the aperture are detected by the optical CCD.

Table 3.1 compares the limiting magnitude attainable by the ATD/NTOT equipped with an enhanced primary mirror and an optical framing camera, and by the Hubble Space Telescope equipped with either the existing second-generation Wide Field/Planetary Camera-2 (WFPC2) or the proposed Advanced Camera (AC). The ATD/NTOT will go nearly 3 magnitudes deeper in the I-band than can the HST with the WFPC2, and will go more than a magnitude deeper than can the HST with the AC. In the assumed bandpass, the ATD/NTOT will have approximately $70 \%$ better resolution than the HST with the AC and some 3.7 times better resolution than the HST with the WFPC2 (for the more commonly used widefield CCDs). These significant improvements would enable an attack on cosmological and cosmogonical problems that are beyond the reach of the HST.

the central peak will lead to problems when trying to track using very faint guide stars. However, if some of the enhancements discussed in Chapter 2 are implemented, then the outlook is very much improved. Box 3.2, "Enhancing Optical Performance," discusses this possibility in more detail.

\section{SCIENCE OPERATIONS FOR THE ATD/NTOT}

Provided that the ATD/NTOT's demonstration of defense technologies is successfully completed, the task group has assumed that there will be a period devoted purely to astronomical tests and observations. Such science operations might last 5 years, a period commensurate with estimates from Lockheed of the probable lifetime of the ATD/NTOT based on a design lifetime of 1 year. The task group has addressed the question of how to carry out the science operations assuming this lifetime. Successful operations for a drastically shorter period might require a very different approach, but a factor-of-two change would not alter the considerations presented here.

\section{The Science Team}

How the ATD/NTOT will be operated during the astronomical phase of its mission is an important issue. During the early planning for what is now called the Hubble Space Telescope, a committee of the National Academy of Sciences met at Woods Hole to consider how the telescope would be operated. ${ }^{2}$ NASA's vision of the HST as a Great Observatory, serving a large national and international community, had to be accommodated in the operations plan. The Woods Hole committee recommended the creation of a university-affiliated institute at which astronomers would carry out the HST's science operations. The contract for this Space Telescope Science Institute was awarded to the Association of Universities for Research in Astronomy, and the institute was built on the Homewood Campus of Johns Hopkins University.

The task group believes that the Woods Hole principle of using astronomers for science operations is a good one and should be followed for the operation of the ATD/NTOT. However, because of the need to minimize the costs of science operations, it will not be possible to operate the 4-meter telescope as an observatory that executes a large number of programs from a community of guest observers.

Rather, the task group envisions the appointment of a principal investigator and science team that will have responsibility for executing a science program and determining the suitability and limitations of ATD/NTOT 
TABLE 3.1 Limiting Magnitudes for the ATD/NTOT and the HST

\begin{tabular}{|c|c|c|c|c|}
\hline Telescope & $\begin{array}{l}\text { Strehl } \\
\text { Ratio }\end{array}$ & $\begin{array}{l}\text { FWHM Net } \\
(\operatorname{arcsec})\end{array}$ & $\begin{array}{l}\text { DQE } \\
(\%)\end{array}$ & $\begin{array}{l}\text { Limiting I } \\
\text { Magnitude }\end{array}$ \\
\hline ATD/NTOT & 0.8 & 0.040 & 50 & 29.4 \\
\hline HST/AC & 0.9 & 0.070 & 50 & 28.3 \\
\hline HST/WFPC2 & - & 0.150 & 10 & 26.6 \\
\hline
\end{tabular}

NOTE: Comparison between the full width at half maximum (FWHM), detective quantum efficiency (DQE), and estimated limiting magnitudes of the ATD/NTOT equipped with both an enhanced primary mirror and an optical framing camera and the Hubble Space Telescope (HST) equipped with either the second-generation Wide Field/Planetary Camera (WFPC2) or the proposed Advanced Camera (AC). The calculated values assume a 2400 -second exposure through an HST F814W filter; the sky background is $22.7 \mathrm{~V}$ magnitudes per square arc sec; and the limiting magnitude is the I magnitude of a star, of effective temperature $4700 \mathrm{~K}$, which has a signal-to-noise ratio of 10 through an aperture that encircles half the light.

technology for the next generation of large space telescopes. The science team will be responsible for the science operations defined below. In the task group's view, the science phase of the ATD/NTOT mission will have more in common with a small- or medium-class explorer mission than with the HST. At the outset of the program, somebody must oversee definition of science policies and goals and selection of the science team.

The scientists on the mission team will have two responsibilities: (1) adequate definition of the optical assembly so that it is capable of carrying out the astronomical technology demonstrations (see Chapter 4) and does not compromise the astronomical observations, and (2) definition of a science program to be performed after the technology demonstration. This latter task includes consideration of appropriate filters and advice on detectors and optical coatings.

The import of these decisions for the success of the science mission necessitates the inclusion of at least an advisory group of scientists early in the planning of the mission, before design constraints are frozen. It is understood that NASA and the space science community are not the primary customers of this mission but are included as partners to the extent that changes do not add unduly to the cost or change the capabilities of the ATD/ NTOT's demonstrations of technology for DOD.

The composition of the science team should not be static. Rather, it should change as the mission evolves from one phase to the next. At the outset, particularly in the planning phases, the team should consist of astronomers with expertise in the design of large telescopes. The task group emphasizes that the astronomical community has developed considerable experience in the design and building of large astronomical instruments. Astronomers who have been involved with such projects should be included on the team. Additionally, it is essential to select a team of astronomers early in the mission on the basis of proposed scientific activities, so that scientific goals can be kept in mind during the design phase.

The astronomers responsible for design oversight and devising initial science programs would also be responsible for formulating and conducting the technology demonstrations outlined in Chapter 4 , as well as for defining what calibrations are necessary for the instruments and the needed calibration interval. The initial team would start the science projects, including overseeing the sequencing of the observations and the verification of science quality.

If the demonstrations of the astronomical technology are successful and if the ATD/NTOT survives beyond its 1-year design lifetime, as is expected, the task group recommends that NASA select additional members of the science team at approximately yearly intervals to ensure that the mission is always pursuing current, important astronomical questions. It is probable that these new members would replace some of the existing team. It is recommended that sufficient overlap of team members be employed so that efficiency of the sequencing is maintained.

The task group also suggests that the Clementine model be followed with respect to data rights. In other 
words, there should be a short proprietary period while the science team prepares data for archiving and for dissemination to the science community in a timely manner.

\section{Science and Spacecraft Operations}

The task group sees a natural division between what it refers to as science operations (defined below) and operation of the ATD/NTOT spacecraft, telescope, and instruments. During the initial DOD phase of the mission, the latter operations will be done by a command center under contract to the DOD. The task group assumes that this command center will continue to operate the telescope and science instruments during the subsequent science phase.

Based on its experience with comparable military spacecraft, Lockheed estimates that the ATD/NTOT command center will require a staff of 46 people during the astronomy phase. For comparison, the Clementine operations staff had 51 full-time equivalents (FTEs) (excluding 11 FTEs devoted to mission science planning and scheduling) and 15 part-time FTEs. ${ }^{3}$ The HST command center at NASA's Goddard Space Flight Center is staffed by several hundred FTEs. But since the HST is a long-duration mission, many of the FTEs allocated to it are responsible for planning instrumental and software upgrades and preparing for future servicing missions, activities that have no counterparts in a short-duration mission such as the ATD/NTOT.

The first task of the science team is to plan a detailed science program. The program must then be turned into a detailed schedule that can be used by the operations center to produce command loads for the spacecraft and telescope. As the program is executed, considerable bookkeeping must be done to verify that all or part of the observations have been made. Calibration targets must be selected and observed, and the calibration data must be inserted into a data pipeline in an appropriate format. The observations of science targets must be calibrated by going through a data-reduction pipeline and must be distributed to members of the science team and archived for public access. The sum of these tasks - the science operation-are the responsibility of the science team.

\section{Cost of Science Operations}

Although it proposes that a principal-investigator-led science team be responsible for science operations, the task group has estimated the cost of science operations based on experience at the Space Telescope Science Institute (STScI), as projected for the ATD/NTOT mission. The factors it has identified as strong cost drivers are ordered below by approximate impact on operations; factors with the largest impact are listed first.

1. The number of observers served. There is a huge difference in the cost to support a guest observer program versus one with a few knowledgeable co-investigators. A large number of guest observers increases initial costs and greatly increases recurring costs.

2. The number of distinct science programs carried out. There is a substantial overhead cost for planning each program, generally requiring interaction between the operations staff and the observers. Many small programs require more work than a few large ones.

3. The number of scientific instruments and their complexity. There is a large potential cost to document, schedule, command, and analyze data from complex instruments with many different features and operating modes. This factor affects both initial costs and ongoing operations costs.

4. The number of different detectors and calibration flows. These drive the costs of calibration and calibration software.

5. Requirements to observe targets of opportunity. Control centers are most efficient if they can plan well in advance and operate primarily on a one-shift basis. Requiring responses to targets of opportunity drives the techniques for commanding the spacecraft and thereby adds to the cost.

6. Requirements to observe moving targets. Observing moving targets requires extra software for scheduling and computing the positions of objects. For planning observations of moving targets, the interface between the project and the astronomer should be set up so that most of this additional effort could be borne by the astronomer, 
who would be highly motivated to carry it out in the most efficient manner. If not approached in this or a similar manner, a requirement to observe moving targets could become a prime area for unplanned cost growth.

7. The fraction of data collected per orbit that can be stored on-board. If there is sufficient capacity to store data on-board, the scheduling process can ignore data volume, leaving schedules for data downlinks to the control center. If there is insufficient on-board storage capacity, the scheduling process must consider data rates and data volume while scheduling, driving up both the initial and recurring costs.

8. Scheduling constraints. The following constraints have approximately equal impact: the type of orbit; Sun, Earth, and Moon viewing constraints; requirements for time critical observations; and constraints on spacecraft roll angles. The complexity of the constraints drives the initial costs for the scheduling system: the more complex and constrained the actual observing programs, the longer the staff needed to do the scheduling.

9. The complexity of planning for guide-star acquisitions. Selecting guide stars is generally automated, and so it drives the initial costs of the ground system but is a minor driver of recurring costs.

Although the task group thinks that many of the cost drivers such as observing targets of opportunity and observing moving targets are scientifically desirable, it makes the following assumptions in order to estimate a lower limit to the cost of science operations:

- The telescope will be in a 12 -hour Molniya orbit;

- The telescope will acquire guide stars autonomously;

- Sun, Earth, and Moon constraints will be comparable to those of the HST;

- There will be minimal support for time-critical observations, observing targets of opportunity, or observing moving targets;

- The telescope will have sufficient on-board memory to store the data from two orbits; and

- There will not be a guest observer community.

Any of the above assumptions can be relaxed, but doing so will increase the cost of science operations. For example, if the telescope is in low Earth orbit and operated with HST capability and a large guest observer community, the cost of science operations will be a significant fraction of the cost of HST science operations.

For the purposes of evaluating the likely costs of supporting such a mission, the task group has used estimates from the STScI. While other approaches to supporting the mission could conceivably be used, drawing on the substantial investment in support activities at the STScI would allow substantial savings in cases where to do otherwise would be to "reinvent the wheel." In addition, some services could be provided quite effectively at marginal cost (as is normally done, for example, with archives where the substantial investment by NASA in hardware and software is utilized for a number of programs).

The task group estimates that the task of planning the science program will require the equivalent of one fulltime astronomer. It assumes that the schedule of science observations will be created with the software developed at the STScI to schedule the HST, namely, the artificial intelligence system called "Spike." Spike is being used to schedule NASA's Extreme Ultraviolet Explorer and Japan's Advanced Satellite for Cosmology and Astrophysics and is being considered for NASA's X-Ray Timing Explorer and Far Ultraviolet Spectroscopic Explorer. Thus it is a system with demonstrated utility for smaller science missions.

Using Spike, two or three people could schedule the ATD/NTOT's scientific observations. The precise number will be determined by the requirements of the spacecraft and telescope control center. Based on information supplied by the STScI, the task group estimates that Spike could likely be converted to the scheduling specifics of the 4-meter telescope for about $\$ 100,000$. The exact amount will depend on the mission parameters of the spacecraft.

The analysis here is based on the assumption that the science team will contract with the STScI for the use of its existing data pipeline. The present HST pipeline handles images from four $800 \times 800$ CCDs. By 1997 the pipeline will calibrate images from the Space Telescope Imaging Spectrograph's (STIS) $1024 \times 1024$ CCDs, and infrared images from the $256 \times 256 \mathrm{HgCdTe}$ arrays of the Near Infrared Camera and Multi-Object Spectrograph (NICMOS).

The pipeline could be modified to accommodate optical and infrared images from the ATD/NTOT for a cost 
TABLE 3.2 Estimated Annual Costs in Full-Time Equivalents (FTEs) for BMDO 4-meter ATD/NTOT Science Operations

\begin{tabular}{|c|c|c|c|}
\hline \multirow[b]{2}{*}{ Task } & \multicolumn{2}{|c|}{ Science Team } & \multirow[b]{2}{*}{ Total FTEs } \\
\hline & Optical & Infrared & \\
\hline Planning the observing program & 0.5 & 0.5 & 1.0 \\
\hline Scheduling the scientific observations & 1.5 & 1.5 & 3.0 \\
\hline Monitoring data quality and calibrations & 2.0 & 2.0 & 4.0 \\
\hline $\begin{array}{l}\text { Using Space Telescope Science Institute } \\
\text { pipeline for data reduction }\end{array}$ & 0.5 & 0.5 & 1.0 \\
\hline Total Science Operations & & & 9.0 \\
\hline
\end{tabular}

of approximately $\$ 200,000$. Approximately two people for each of the two instruments (infrared and optical) would be needed at the STScI to monitor science data quality, plan calibration observations, reduce the calibration observations, produce the calibration files, and deliver the reduced data to the science teams. These four astronomers could either be members of the science team or STScI staff working under contract to the science team. The cost of contracting to use the STScI modified pipeline are estimated to be one FTE per year.

The cost of modifying the STScl data archive to accept ATD/NTOT images would be approximately $\$ 100,000$. There would be a relatively small operational charge for optical disks, scaled by the volume of data collected by the telescope. The four calibration scientists would be responsible for delivering the data to the archive and ensuring that the data were archived correctly. The estimated costs in FTEs for science operations are summarized in Table 3.2 .

The annual estimated effort for science operations is 9 FTEs. This estimate can be compared to the 11 FTEs in the Clementine mission devoted to mission science planning and scheduling. For comparison, the STScI schedules some 300 guest observer (and Investigation Definition Team) proposals a year on the HST's four multimode science instruments. Approximately 50 FTEs are required to process and schedule the proposals, and another 10 FTEs are needed to maintain the processing and scheduling software. Approximately 12 people are required to process the data through the data-reduction pipeline and put the data into the data archive. These numbers do not include the approximately 20 FTEs required to support the instruments and instrument calibrations, nor the science data aides who support visiting astronomers at the STScl.

In summary, the task group estimates that 9 FTEs will be needed for ATD/NTOT science operations. The one-time cost for software conversion is approximately $\$ 400,000$. It has not estimated the cost of writing or modifying software for data analysis, or the cost of supporting the science team for analysis of the science data.

In the task group's opinion, these estimates of the costs of science operations are a lower limit to the operations cost. Experience shows that unanticipated problems will increase costs. Additional scientific requirements will also increase costs.

\section{SUMMARY}

The preceding sections suggest some clear directions for astronomical use of the ATD/NTOT beyond the demonstrations of technological capability. Large surveys are an appropriate use of the ATD/NTOT because they can be carried out at minimal operational cost. Furthermore, surveys make repeated use of a single mode and require only a small team of scientists. Such observations are not an optimal use of major facilities such as the HST, whose high versatility and complex instrumentation can support a wide community of users with diverse needs. In the context of surveys with the ATD/NTOT, which will necessarily sample the sky sparsely, guest observers could, perhaps, be accommodated to the extent that they want observations of the same general type as are being made for the surveys but that are pointed at other parts of the sky to view particular targets. Adding to 
the survey a target list that requires no extra calibrations, provided the observations are not time critical, adds minimal extra cost to the operations.

Emphasis should be given to programs for which access to space significantly reduces the sky background for observations and to programs for which either high spatial resolution or large collecting area is important. The ATD/NTOT thus has a major advantage over other facilities for the following kinds of programs:

- Programs in the near infrared ( 2 to 4 microns), where the difference in the sky background is reduced by several orders of magnitude (largely through reduction of $\mathrm{H}_{2} \mathrm{O}$ and other tropospheric species);

- Programs in the far red $(>0.7$ micron), where the sky is reduced by up to one order of magnitude (OH emission); and

- Programs that depend on the contrast between a point source and its neighborhood (either the sky background or its own astronomical environment) or that require subarc-second spatial resolution to reach interesting spatial scales or that are photon-starved, that is, receive little attention with the HST.

\section{REFERENCES}

1. Thompson, R.I., "NICMOS: A Second Generation Infrared Instrument for the Hubble Space Telescope," Advances in Space Research 13(12):509-519, 1993.

2. Space Science Board, National Research Council. Institutional Arrangements for the Space Telescope, National Academy of Sciences, Washington, D.C., 1976. Also see, Space Science Board, Institutional Arrangements for the Space Telescope: A Mid-Term Review, National Academy Press, Washington, D.C., 1985.

3. Space Studies Board, National Research Council, Lessons Learned from the Clementine Mission, National Academy Press, Washington, D.C., in preparation. 


\section{Astronomical Technology Demonstrations}

Assuming that the ATD/NTOT flies as a demonstration of technology for future DOD applications, it is most appropriate that the first astronomical goal should be a similar demonstration, or at least a test, of technological capability for astronomical applications. Based on the information at its disposal, the task group believes that many, but obviously not all, of the activities in support of this goal can be carried out during DOD's technology demonstrations. This chapter enumerates some of the many possible demonstrations and tests of capabilities that would be of interest to astronomers.

Some of the tests suggested here are essential to validate the ATD/NTOT technology for use in future space astronomy missions. Other tests are desirable but not essential. A number of the suggested tests are directly related to the possible scientific programs that could be conducted in an extended mission, and others are not. Some of the demonstrations can be carried out entirely within the baseline mission, whereas others require one or more of the performance enhancements discussed in Chapter 2.

The task group would like to see all of its suggested enhancements to the baseline ATD/NTOT implemented. The critical ones are improving the surface of the primary mirror and adding the optical framing camera. Nevertheless, the task group recognizes that none of them may be financially feasible, and some may even be incompatible with the still-changing goals of DOD's mission. If none can be made, the baseline mission (as understood in mid-1995) is still capable of demonstrating many important technological capabilities that are of interest to astronomers.

Clearly, the task group's list of possible demonstrations is not intended to be exhaustive. Rather, it is an initial list from which tasks can be added or deleted as the financial constraints and programmatic goals of the ATD/ NTOT mission become clearer.

\section{DEMONSTRATIONS DURING THE BASELINE MISSION}

\section{Use of Active Optics}

The most fundamental demonstration for astronomical purposes is to show that large mirrors can be refigured on orbit. The ability to sense a wavefront and close the feedback loop to control the shape of the mirror is critical to using the ATD/NTOT's technology in future astronomical missions.

As part of this demonstration, various approaches to closing the loop should be explored. Techniques to be 
explored should range from autonomously closing the loop on the spacecraft to deliberately varying the focus in order to attempt phase retrieval on the ground and uploading of new transfer matrices for the feedback loop. The interferograms from the wavefront sensor must be recorded and returned to the ground to enable thorough analysis.

\section{Image Quality}

A top priority is to evaluate the quality of the images. With the baseline equipment, evaluation will be primarily indirect, based on the output from the optical wavefront sensor. The quality of the images must be evaluated under a variety of conditions, including various positions in the field of view that can be reached through the fast steering mirror and a variety of thermal conditions.

To explore the effects of thermal environment, the image quality should be studied under a variety of orbital configurations. If the DOD phase of the mission is carried out in low Earth orbit, then these tests should first be done in the low Earth orbit and then repeated in the Molniya orbit. In addition, the image quality should be assessed after the DOD mission during a period in which the telescope is pointed so as to let it cool to the minimum possible operating temperature.

In addition to evaluating the images with data from the wavefront sensor, it is also important to evaluate actual images from both the InSb array and the fine-tracking array. Although neither of these detectors is ideal for analyzing the images, they do provide important information about the images to supplement that available from the wavefront sensor, and they also provide an end-to-end test of the system.

\section{Thermal Background}

The next critical test is to determine the thermal emission characteristics of the telescope and other components of the system. Like the test for image quality, this demonstration should be done both in the initial orbit and in the Molniya orbit to understand the changes in thermal background as a function of the orbital characteristics. The task group's initial estimates are that, when in low Earth orbit, the telescope's thermal emission will be too high for deep-infrared observations. However, a Molniya orbit, in which substantial passive cooling can occur, should allow such observations.

The ATD/NTOT's design has not been optimized for thermal control. In addition, it is very different from traditional infrared-optimized telescopes on the ground. Thus, there are no detailed analyses available that can be used even as a guideline for estimating its performance. If designed from the outset for low thermal background, it would likely be quite different from the baseline system described in Chapter 2. Thus determining the thermal emission characteristics is critical for understanding the astronomical capability of the ATD/NTOT. Low thermal background is essential for carrying out the near-infrared cosmological survey discussed in Chapter 5.

Evaluation of the baseline telescope's thermal environment would be carried out using images obtained with the InSb array. It is not clear to the task group whether this detector will have sufficient sensitivity to fully characterize the telescope's emission under all possible circumstances. It will certainly be adequate for characterizing the emission whenever the system is, in some relative sense, warm. Furthermore, the thermal background seen at the focal plane is likely to vary as the fast steering mirror follows the motion of the target with the field of the telescope. These variations must be evaluated in order to assess the limits that they place on detecting faint sources at infrared wavelengths.

\section{Field Distortion Stability}

Since the ATD/NTOT is fundamentally different from most ground-based telescopes, the stability of its field distortion must be evaluated. This factor is important because it directly affects the ability to make long exposures. Optical stability also affects the ability to detect moving targets and to register successive images of the same field to remove cosmic-ray hits (something which may be essential because the ATD/NTOT's eccentric orbit will take it well outside the shielding of Earth's magnetosphere).

A particular concern is that the field distortion is very likely to change as the fast steering mirror moves the 
field of view to counter the spacecraft's motion. Furthermore, the distortion may change as the actuators respond to remove fluctuations in the shape of the primary mirror. The distortion of the field has not been assessed, and so it is impossible at this point to evaluate the changes in distortion.

Much of the evaluation of field stability can take place in prelaunch computer simulations. The ultimate test will, however, be done in space. Distortions should be evaluated during the DOD phase of the mission in low Earth orbit as well as during the astronomical phase in a Molniya orbit.

\section{Orbital Maneuvering}

The DOD's technology demonstrations require that the ATD/NTOT be highly maneuverable and be able to slew rapidly from one point on the sky to another. This ability combined with the substantial reserves of hydrazine (i.e., beyond that needed for station keeping and dumping angular momentum) carried by the baseline mission raises an interesting possibility. It should be possible to maneuver the spacecraft so that it is in the correct position to observe a spatially localized event such as a stellar occultation (e.g. by an asteroid or by a planetary atmosphere). Like solar eclipses, the tracks of these events are generally visible in regions considerably smaller in size than the Earth; thus mobility is key to observing them. Moreover, the much larger geometric cross section presented by the Molniya orbit will allow the ATD/NTOT to potentially intercept more such events than are possible from Earth-based facilities or telescopes in low Earth orbit.

The ability to maneuver a space telescope so that it is in the right place at the right time to rendezvous with an occultation path would be as revolutionary as the advance realized in the 1970s and 1980s by exploiting the mobility of NASA's Kuiper Airborne Observatory-a capability that led to the discovery of the rings of Uranus and Pluto's atmosphere. A 4-meter space telescope in a Molniya orbit could observe occultations inaccessible to ground-based observatories or telescopes in low Earth orbit, greatly increasing the potential application of the occultation technique.

Successful demonstration that an orbiting telescope could be maneuvered to record occultations unobservable from Earth would open the way for comprehensive investigations of the occultations of virtually any chosen body in the solar system (including the possibility of measuring diameters of Kuiper Disk objects).

Since most occultation tracks are roughly parallel to Earth's equator, each such track would cross a highly inclined orbit, such as the ATD/NTOT's Molniya orbit, somewhere and thus could be intercepted by changing the phase of the telescope in its orbit. If major changes in orbital energy are discounted, few additional events would be reached by adjusting the eccentricity or inclination of the ATD/NTOT's orbit. If a truly spectacular occultation could be reached by more radical orbital maneuvers, then the impact of such changes on other operations would have to be carefully assessed.

The orbital variations needed to adjust the ATD/NTOT's orbital phase so that it would arrive in line with the body to be studied and a bright star might typically require velocity changes of a few meters per second (the amount of fuel required for a velocity change of $10 \mathrm{~m} / \mathrm{s}$ is $0.5 \%$ of the mass of the spacecraft, that is, about $30 \mathrm{~kg}$ ). Although time critical, a sequence of maneuvers for a given event could be planned well in advance, with the motions specified by Earth-based or space-based astrometric measurements.

The largest maneuver required would be an advance (or retardation) in orbital longitude of the telescope by 180 degrees over a period of 2 months. For an orbit with a period of 12 hours, this maneuver would require a velocity change of $5.4 \mathrm{~m} / \mathrm{s}$. If the accuracy of this maneuver were only $10 \%$, then one would "home in" on the correct orbit by two more maneuvers of the same magnitude - the first, 6 days prior to the occultation and the final one, 14 hours before. The final accuracy of the ATD/NTOT's location, in this hypothetical case, would be some $85 \mathrm{~km}$. This would be sufficient to position the ATD/NTOT close enough to the center of the shadow of, for example, Triton or Pluto to probe into their atmospheres as deeply as is possible during an occultation.

Imaging arrays are now the preferred tool for observing occultations, because the data they provide enables the construction of sophisticated models to remove the effects of background light. Contrary to the case for lunar occultations, no additional information is gained in planetary occultations by the use of kilohertz recording rates, because Fresnel diffraction produces a blur on a time scale of

$$
\frac{\sqrt{\lambda D}}{v} \text {. }
$$


For a typical asteroidal occultation observed at 0.75 micron, the distance $D=1.5 \mathrm{AU}$ and the velocity $v=20 \mathrm{~km} / \mathrm{s}$ imply a time scale of $0.02 \mathrm{~s}$. In addition, rather than reading out the full array, subarrays are read as needed. Thus, occultation data recording rates are typically 10 to $50 \mathrm{~Hz}$, and the recording should be synchronized with absolute time and spacecraft position. Since the high-speed array detectors planned for the military mission would provide simultaneous occultation light curves at visible and infrared wavelengths at these rates, no special changes to the baseline plan appear to be necessary for this astronomical technology demonstration other than the on-orbit maneuverability. Successful demonstration of this maneuvering capability would, for example, permit the program to investigate global change on Pluto and Triton as described in Chapter 5.

\section{Very Long Exposures During a Single Orbit}

Being able to reach faint limiting magnitudes is a major advantage of large telescopes. Observing the faintest objects requires long integration times and correspondingly long times on-source.

Performing long integrations is a very inefficient use of telescopes in low Earth orbit (e.g., the HST) since half of each orbit is occupied with Earth occultation. Moreover, the target must be reacquired during each orbit. In practice, only 30 to 40 minutes of each of the HST's 90 -minute orbits is actual observing time for a single, very long exposure. Thus it would take 15 to 16 orbits, or 24 hours, to achieve an 8 -hour integration. In contrast, this same observation could be performed in a single, 12-hour Molniya orbit.

Even with ground-based telescopes, 8 continuous hours of observation is rare, unless the observation is done at large air masses. Only the International Ultraviolet Explorer in its geostationary orbit can match or exceed the efficiency of the ATD/NTOT's baseline Molniya orbit.

Long integration times have other uses besides just pushing to faint limiting magnitudes. They are preferred when looking for rotational or pulsational periodicities in objects because aliasing problems are reduced if interruptions in the data stream are minimized. Typical observations from the ground suffer severe aliasing problems since an object is observed only at night. For ground-based observations, this problem can be overcome only by highly coordinated observing programs involving many telescopes around the world.

With a Molniya orbit and on-board data storage for times when there was no direct communication with a ground station, it would be possible to achieve almost continuous coverage of an object, eliminating much of the aliasing. The removal of aliasing is especially critical in studies of pulsation modes in stars.

\section{Testing Modes for Operations and Scheduling}

Operations and scheduling of orbiting telescopes can be and have been accomplished in many different modes. The different approaches to key observational issues discussed in this section include the following:

- Observational programs defined by science teams and individual observers;

- Block scheduling and queue scheduling;

- Operations from a dedicated operations center and from a private institution (such as a university); and

- Response to targets of opportunity.

Although it has urged that routine operations of the ATD/NTOT be conducted in the first of these modes (that is, by a science team), the task group notes the possibility of experimenting with other modes that might be important for certain types of observations on future missions.

The choice among these options should be considered in terms of cost, "scientific efficiency," and the degree of autonomy granted to users (within the bounds of safely operating the facility). However, quantitative comparison of these factors, especially the latter two, is difficult, if not impossible. For example, scientific efficiency could be gauged in terms of quantifiable measures, such as on-target time or the number of research publications produced by users of the facility. High marks with respect to these measures, however, could ignore missed opportunities for discoveries that might have resulted from programs not carried out by the facility because they did not fit into a more rigid program.

As discussed in Chapter 3 , the task group anticipates that the operations of this telescope will generally be 
under the control of a science team rather than a support group charged with meeting everyone's needs. It also expects that in an extended mission this science team might evolve. Although the task group does not anticipate that the ATD/NTOT will ever be operated primarily as a facility for guest observers, some experimental changes could be made in the mode of operation to enable important, direct comparison of different operational philosophies.

One key point of comparison is the way in which the telescope responds to targets of opportunity (TOs). Responses might range from never deviating from a planned sequence (almost certainly the least expensive approach) all the way up to attempting to respond to a variety of TOs within hours of discovery. Typical targets of this type might include novae and supernovae as well as comets and an occasional, particularly interesting asteroid, such as one passing unusually close to Earth. These might be called externally discovered TOs.

There are also what might be termed internally discovered TOs, for example, the detection of an anomalous event during monitoring of data being collected in survey projects. The possibility of such events suggests the need to investigate the efficiency of several different preplanned responses. In other words, a survey for supernovae, for example, might have a preplanned response to the discovery of a supernova that would stop the survey and monitor the light curve of the supernova.

Past space missions have traded the operating efficiency of planned programs to respond to TOs in a variety of ways. These range from immediate hands-on control, such as the ability to reprogram the International Ultraviolet Explorer in real time, through a more typical response involving a lag of several hours to days, on down to delays of several weeks, as when the HST is used to respond to less urgent TOs.

A variety of operational modes can be achieved with the telescope under the control of the science team that is responsible for implementing all observations, including those of TOs. Such a team can determine the necessary trade-offs on the basis of the expected scientific return, provided, of course, that the team (or at least the team in charge of operations for a particular time period) has an interest in TOs. The key point is to put the workload for implementing a particular capability on the users, that is, the science team, for implementing observations of TOs. A key test of the capability to respond to externally discovered TOs would be to carry out follow-up observations on Earth-approaching objects discovered by the Spaceguard ${ }^{1}$ or equivalent surveys of near-Earth objects. It should be kept in mind that other telescopes will be available for follow-up observations of TOs, so that observations of such targets with the ATD/NTOT should in any case be limited to tests of responsiveness and to observations that cannot be done with other telescopes such as the HST.

\section{ASTRONOMICALLY ENHANCED DEMONSTRATIONS}

Although the astronomical community can learn much from those demonstrations that can be performed with the baseline ATD/NTOT, certain mission enhancements offer improved capabilities for astronomical technology demonstrations. The task group notes that, as always, a cost/benefit analysis must be performed prior to enhancing the baseline mission. The following are among the technology demonstrations that could be performed with enhancements to the baseline ATD/NTOT.

\section{Enhanced Image Quality}

The single most valuable enhancement for testing the applicability of the ATD/NTOT's technology to astronomy is improving the figure of the primary mirror. Figuring the primary to an accuracy of better than $20 \mathrm{~nm}$ rms would reduce to roughly $50 \%$ its contribution to the total error budget. Although still significant, this level would allow investigations of the effects of other error sources more relevant to the ATD/NTOT's unique technology. These additional sources include the tracking system, the fluctuating thermal load, the varying field distortion as the fast steering mirror moves the field, and so on. The addition of an optical framing camera would lead to significant additional information about the performance of the telescope.

As noted in Chapter 2, the fine-tracking camera (if equipped with line-transfer CCDs) does not completely sample the point-spread function (PSF) and does not allow the long exposures typically used in astronomical observations. The InSb array, on the other hand, will fully sample the PSF but only at a relatively long wavelength such that the larger diffraction limit is a significant factor in the PSF. Thus the optical framing camera would lead to a major improvement in the ability to evaluate the PSF under realistic astronomical conditions. 
Long exposures at the shortest practical wavelength (approximately 0.5 micron) would provide the best, complete, end-to-end test of the ATD/NTOT's technology. Such exposures would also serve as a direct test of the telescope's utility for astronomical imaging. Long exposures would allow a complete characterization of all aspects of the system at all spatial frequencies, including effects at spatial frequencies higher than those sampled by the wavefront sensor.

While image quality must be tested and calibrated by observing point sources, the real demonstration of quality suited to astronomical purposes will be in the ability to separate a point source from a close, fainter neighborhood. Ideal as a test for this task are attempts to resolve the galaxies surrounding quasars and cometary nuclei from their comae.

\section{Thermal Background}

In the baseline mission, the thermal background would be evaluated using the InSb array. It is not clear to the task group whether this detector would be limited internally by its own noise or externally by the emissivity of the optics in the infrared beam. Enhanced cooling of the InSb array, required for sky-limited astronomical performance, might be an important addition for evaluating the thermal performance of the system if the telescope and its optics are allowed to cool to a sufficiently low temperature.

\section{Field Distortion Stability}

The addition of an astronomical framing camera would allow several additional measurements that are related directly to the astronomical projects discussed in Chapter 5 and that also can demonstrate stability of the field, or at least the ability to calibrate the distortions. The distortion of the field is naturally a factor in all astrometric observations, but it is also a very important factor in co-adding images to remove artifacts caused by cosmic rays. There should certainly be a series of astrometric tests on rich stellar fields, but there are other possible tests that would also represent a real advance in astronomical capability, for example, determination of the geocentric parallax of objects in the Kuiper Disk.

Once a suspected Kuiper Disk object is discovered, its orbit must be determined to see if it is really a member of the cometary reservoir located beyond Neptune's orbit. This is a laborious task for ground-based telescopes because it entails many months of astrometric observations to define an orbit accurately enough to determine its distance to better than $10 \%$. Furthermore, the follow-up observations must be made soon enough (within weeks) to ensure that the object is not lost.

There is a faster way to measure the distances to suspected Kuiper Disk objects. An object at 40 AU has a parallax of about 0.5 arc sec (about 5 pixels) when viewed from one side of the HST's orbit to the other. This is a difficult observation since it requires being able to produce a good secondary reference frame for each image. In a Molniya orbit, the baseline between apogee and perigee is much longer than that for the HST and the corresponding parallax is 1.8 arc sec. Coupled with the higher spatial resolution of the ATD/NTOT, this displacement translates to 60 pixels, and the determination of geocentric parallax becomes straightforward.

Thus the combination of a larger orbit and smaller PSF leads to an order-of-magnitude improvement in the ability to measure parallax. These factors, in turn, lead to an improvement by two orders of magnitude in the time needed (days instead of a year) to determine an orbit for an object in the Kuiper Disk.

Of course, many other more typical astronomical applications of astrometry could also be used as a test of the field stability and astrometric performance of the telescope. A particularly interesting possibility is to directly measure motions of stars and emission-line sources in star-forming regions using the infrared detector.

\section{Photometric Stability}

Although the ATD/NTOT's instruments are imagers, they may also be used to make accurate photometric measurements of objects. One important test of the ATD/NTOT's capabilities will be the precision and accuracy of such measurements, both on short time scales and over long time periods. However, it should be noted that 
many photometric studies do not require absolute accuracy but can be accomplished in a differential sense with high precision. Unless the point-spread function is highly oversampled, photometry does require a camera without "gaps," such as the masked alternate rows in the fine-tracking camera's line-transfer CCD, in order to collect all the light from a source. The inclusion of an optical framing camera would circumvent this problem.

An excellent example of the necessary level of precision is seen in the field of stellar seismology. In these observations, astronomers search for radial and nonradial pulsational modes in stars by looking at the variation of the light with time. The amplitude of the variations and the relevant time scales are functions of the properties of the star. The Sun, for example, has oscillations with periods between 3 minutes and 27 days, but relatively small amplitudes.

Observations of much more distant solar-type stars will require rapid sampling at high precision, an approach that probably will not be possible to high enough precision with an imaging detector. However, if a star is mostly degenerate and has a large effective gravity (e.g., white dwarfs and neutron stars), the amplitude of the pulsations will be as large as a few hundredths to tenths of a magnitude. CCDs are capable of accuracy of this order even sampling at the $20-$ to $60-\mathrm{Hz}$ rate at which white dwarfs and neutron stars pulse (though this requirement may compromise the low-read-noise performance of the CCD system, and so some trade-offs may be needed).

Observations of this type are typically made in a differential sense, that is, by comparing the target star with nearby field stars so that drifts in instrumental sensitivity with time are not important. A critical problem with ground-based studies of white-dwarf pulsations is the aliasing introduced by observing only at night. With sufficient control of the photometry, the ATD/NTOT's Molniya orbit could minimize or eliminate much of the aliasing, allowing determination of pulsation modes more accurately and more quickly than would be possible from the ground.

A program to test the photometric stability over time scales from milliseconds to months is an excellent test of the ATD/NTOT's technological capability for astronomical applications. If the photometry is found to be precise and stable, surveys of oscillating stars may become a feasible science project to consider for an extended mission.

\section{REFERENCE}

1. Morrison, David (ed.), The Spaceguard Survey: Report of the NASA International Near-Earth-Object Detection Workshop, NASA, Office of Space Science and Applications, Washington, D.C., 1992. 


\section{5}

\section{Science Projects}

If the ATD/NTOT passes many of the key technological tests outlined in Chapter 4 and can also exceed its design lifetime by a considerable margin, it should have a significant capability for astronomical research. This being the case, what science projects can be conducted with the ATD/NTOT, but not with any other facility currently in development?

The report of the Astronomy and Astrophysics Survey (Bahcall) Committee ${ }^{1}$ emphasized an understanding of origins - the formation of the universe, galaxies, and stars and planets-as an essential theme for astronomy in the 1990s. Similarly, a more recent report by the Committee on Planetary and Lunar Exploration ${ }^{2}$ underscored an understanding of the origins of planetary systems and life as one of the principal motivations for research in the planetary sciences in the period from 1995 to 2010.

Given these endorsements, the task group emphasizes in this chapter some projects that are critical to understanding origins. Furthermore, it emphasizes activities that require large amounts of observing time (i.e., surveys). As explained in Chapters 3 and 4 , surveys are perhaps the minimal-cost mode for operating a large telescope, and they should not be done with complex, versatile instruments such as the HST that serve wide communities of users.

The first project discussed here involves the use of wide-field infrared surveys to directly address the origin of galaxies. The second and third projects address the origin of planets and stars; one looks at primitive planetesimals preserved in the Kuiper Disk beyond the orbit of Neptune, while the other focuses on young stars still in the throes of their birth. The fourth is a possible project for addressing the origin and subsequent evolution of planetary atmospheres by observing global change on Pluto and Triton.

The ability to carry out these projects must, of course, be assessed after the ATD/NTOT is in orbit and its performance has been adequately tested. The infrared galaxy survey, though significantly enhanced by the addition of a large-format framing camera, can be carried out fruitfully so long as the baseline ATD/NTOT performs somewhere near its expected level. The Kuiper Disk survey requires the addition of a framing camera. Moreover, the scientific return from both projects is improved dramatically (by at least an order of magnitude) if the ATD/NTOT is flown with the enhanced mirror suggested by the task group. The young-star project can be carried out in part if the telescope performs nominally, but other aspects of this undertaking require that the pointspread function be very stable and that scattered light is minimized-factors that will not be fully understood until after launch. Although important scientifically, this project is one that becomes appropriate for the ATDNTOT only if the technology performs very well.

The study of global change on Pluto and Triton differs dramatically from the other projects in that it requires 
extensive operational control of the spacecraft. Indeed, conducting this project depends not so much on the ATD/ NTOT's performance as on other factors. Prime among these are the extra operational costs. Nevertheless, if the ATD/NTOT is viewed as a demonstration of technological capability, such an innovative use of a spacecraft represents an interesting challenge.

\section{WIDE-FIELD INFRARED SURVEYS}

The area in which the baseline ATD/NTOT has the greatest promise for making new astronomical discoveries is in deep infrared surveys of the early universe. Several possible observing campaigns are described below.

\section{Galaxies in the Early Universe}

Several lines of evidence suggest that the epoch of galaxy formation lies beyond a redshift $(z)$ of about 1 . For example, the co-moving number density of both quasars and their absorption-line systems changes dramatically for redshifts greater than approximately 1.5. The nature of galaxies associated with distant radio sources, and the nature of the radio sources themselves, also change significantly for redshifts beyond $z \sim 1$.

This has been beautifully exemplified in HST images of the radio source 3C 324 at $z \sim 1.2$ (Figure 5.1). The dramatic examples of interacting and merging galaxies in this image are also seen at lower redshifts, but it is becoming clear that not all galaxies have evolved significantly below $z \sim 1$. Dwarf galaxies at lower redshifts show evidence of substantial evolution, primarily through bursts of star formation. But more massive galaxies appear to be different; the available data suggest that the bulk of their formation occurred at redshifts greater than about 0.8 to 1 . As a class, massive galaxies appear to have undergone little evolution between that time and the present day. Thus the formation or assemblage of these objects must have occurred at a higher redshift.

Unfortunately, extensive studies of such objects with the HST will prove to be very difficult. Because they are expected to have high sensitivity from below 0.4 microns to $\sim 2$ microns, the HST's second- and third-generation imaging instruments, such as NICMOS and HACE, respectively, will clearly provide substantial gains for studying distant galaxies. However, several factors mitigate against the HST for a comprehensive study of galaxies at redshifts of $z \sim 1$ to 5 .

At these redshifts, the optical and near-infrared images correspond largely to wavelengths in the rest-frame ultraviolet. The brightness of a galaxy in the ultraviolet, however, depends dramatically on the amount of star formation and on the quantity and distribution of dust. Surface brightness dimming (through the $(1+z)^{4}$ factor) also has a major impact at these redshifts. The contrast of distant galaxies against the foreground therefore decreases rapidly at redshifts of $z>1$. While quite model dependent, the dilution factor owing to foreground galaxies could be as large as 100:1.

The cumulative effect of all these factors is large and is exacerbated by the HST's small collecting area. Extremely luminous star-burst galaxies with strong near-ultraviolet and ultraviolet fluxes will likely be identified and studied with the HST, but a more complete census of high-redshift objects will require a telescope with greater infrared sensitivity. Typical "young" galaxies are likely to have broadly peaked energy distributions around 0.4 to 0.6 microns, and so the study of these objects is best carried out in the wavelength range $0.5(1+z)$ microns, or $\sim 1$ to 5 microns for such objects in the redshift range $1<z<6$.

The sensitivity of most large, ground-based telescopes is limited for wavelengths greater than 2 microns by their own extremely high thermal background. Only telescopes that are both optimized for the infrared and located in Antarctica have a chance of reaching the limits of the atmospheric background, which even in Antarctica is considerable compared to, for example, the zodiacal background seen from space. Adaptive optics will clearly help these telescopes by increasing the contrast between small sources and the background, but the background remains a dominant source of noise, especially for any extended components of the sources.

Cryogenic space telescopes, such as the European Space Agency's soon-to-be-launched Infrared Space Observatory (ISO) and NASA's proposed Space Infrared Telescope Facility (SIRTF), will be free from any thermal background at the wavelength regions being considered here, but they will face another problem. At the faint magnitudes required for study of these distant galaxies, source confusion is already a serious concern. At a median 


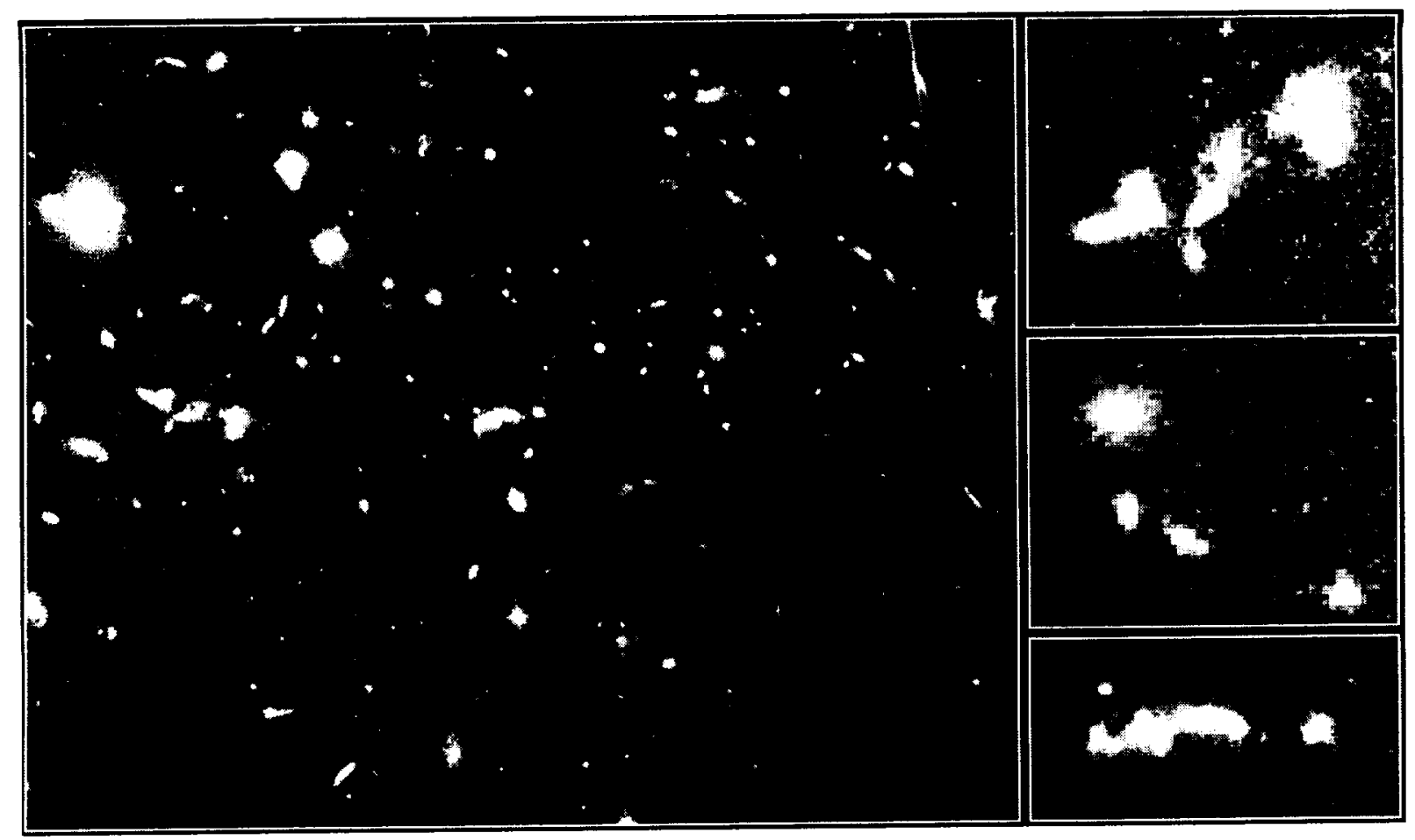

FIGURE 5.1 This deep HST image shows the distant cluster of galaxies around the radio source $3 \mathrm{C} 324$. Very few of the cluster's members are recognizable as normal spiral galaxies. Most have irregular shapes (inset, top) and appear disrupted, possibly due to mergers and interactions. In contrast, the elliptical galaxies present (inset, middle) are remarkably similar to those seen at lower redshifts. The peculiar radio galaxy $3 \mathrm{C} 324$ (inset, bottom) has a redshift of 1.2 . This image, which shows objects as faint as 29th magnitude, is the full field of view of the HST's Wide-Field/Planetary Camera-2. It required an 18hour-long integration, made over 32 orbits between May 11 and June 12, 1994. (Courtesy of NASA's Space Telescope Science Institute.)

redshift of $z \sim 0.6$, there are almost $10^{6}$ background galaxies per square degree. Thus, as seen with a resolution of approximately 1 arc sec, background galaxies cover some 10 to $15 \%$ of the sky. Surveys attempting to reach significantly higher median redshifts will require sensitivities an order of magnitude higher, at which point small space telescopes (like ISO and SIRTF) or large ground-based telescopes without adaptive optics will become seriously confusion limited.

Given that ground-based telescopes experience very large infrared backgrounds relative to the zodiacal minimum (see Chapter 3), it is clear that the optimum approach for galaxy studies is through the use of a large, cooled space telescope. A 4-meter-class telescope in space, with resolutions of the order of 0.1 arc sec, cooled and instrumented for optimum detection in the 2 to $5+$ micron range, would be an immensely powerful tool for comprehensive exploration of the galaxy population in the $1<z \leqslant 6$ range. Although the baseline ATD/NTOT mission is not optimized for low-temperature operations, with modest passive cooling its performance in the 2- to 5 -micron range is still impressive-better, in fact, than anything else currently under development. Moreover, with the implementation of some of the enhancements described in Chapter 2, the ATD/NTOT's performance would be close to that of an ideal system for observing galaxies in the early universe.

The simulations shown in Figure 5.2 compare the expected capability of a 4-meter-class, passively cooled space telescope with that from the ground in the $\mathrm{K}$-band at 2 microns and hence into the background minimum at 3 microns (where the baseline operating temperature of $200 \mathrm{~K}$ provides background-limited performance at 2 microns-but optimum sensitivity at longer wavelengths requires an enhanced design that uses passive cooling to reach $160 \mathrm{~K}$ ). The simulations are also shown for two different values of the deceleration parameter $q_{0}$ (i.e., $\Omega_{0}=$ 


\section{BMDO 4m Cosmology}
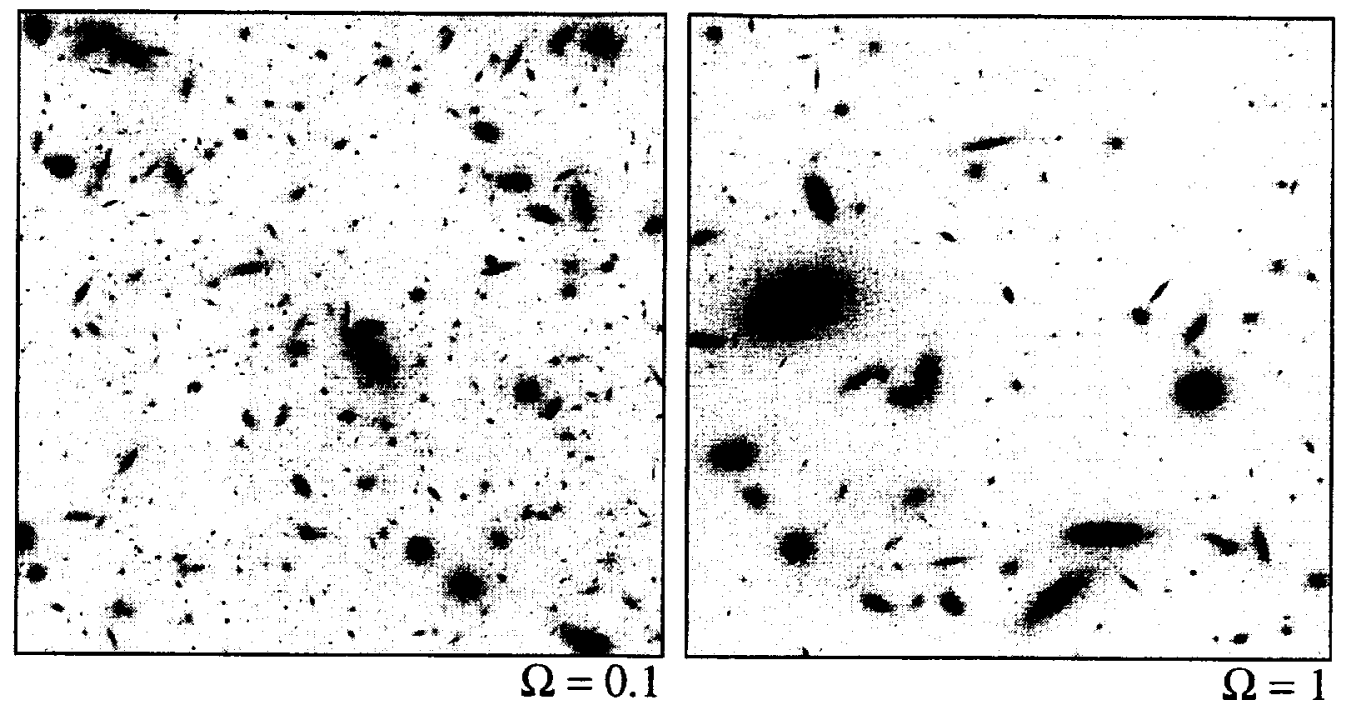

4m Background Limited Imaging at $\mathrm{K}$

$\Omega=0.1$
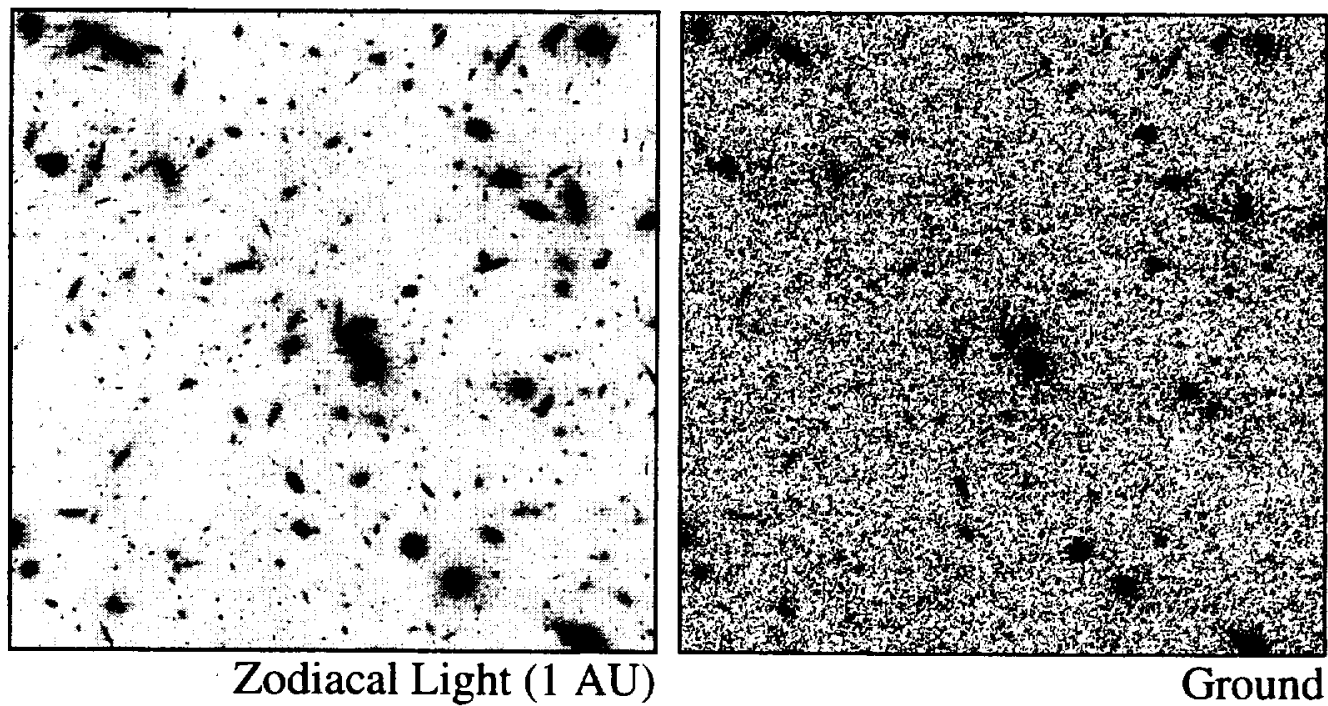

Ground

FIGURE 5.2 Simulations of images of distant galaxies that could be expected from a 4-meter space telescope like the ATD/ NTOT with modest levels of passive cooling appropriate for background-limited K-band imaging (nominally about $220 \mathrm{~K}$ ). The simulations are based on a recent set of evolutionary models (Bruzual, A.G., and Charlot, C., Astrophysical Journal $405: 538,1993)$ where the epoch of formation is assumed to be $z=10$. The bandpass was assumed to be $\mathrm{K}(-2$ to 2.2 microns) and $H_{0}=50 \mathrm{~km} / \mathrm{sec}$ per megaparsec was used. The upper pair of illustrations show what 12-hour exposures would look like for two different cosmologies, an open $\left(\Omega_{0}=0.1\right)$ and a flat $\left(\Omega_{0}=1.0\right)$ model, for images from a 4-meter space telescope. These images do not include a background, though comparison with the lower pair indicates that inclusion of the low space near-infrared background has little effect. The lower pair compare the results from a diffraction-limited 4-meter ground-based telescope (with adaptive optics) with what can be obtained from a 4-meter telescope in space. The difference is dramatic, even in the $\mathrm{K}$ band where the ground-based background is not fully dominated by thermal emission; at 3 microns where the background in space is less, that on the ground would be two to three orders of magnitude greater, essentially wiping out all the objects visible in the panel. (Courtesy of Peter Stockman, Space Telescope Science Institute.) 
1 (flat) or $\Omega_{0}=0.1$ (open), assuming that the cosmological constant $(\Lambda)$ is 0 ). At such high redshifts the counts clearly are influenced very dramatically by the cosmology. Thus, they are a means of discriminating between different cosmological models, although not a very quantitative one because of highly model-dependent uncertainties from merging, star formation, dust, and luminosity evolution.

It is clear that a deep, multifield survey would be of great utility for exploring the early universe. A few regions at high galactic latitude should be selected (free from obvious clustering at lower redshifts and galactic "cirrus"), and a series of integrations carried out in a strip.

Based on a 5-year science mission in which $25 \%$ of the time is spent on the zodiacal-minimum deep (and ultradeep) surveys, some 2800 hours of integrations should be a practical goal for these multifield, multi-waveband observations (assuming an on-target data collecting efficiency of 25\%). For year-round access to low-background regions, and for subsequent ground-based follow up (radio maps, further optical and near-infrared imaging and spectroscopy, and so on), a nominal goal would be to observe three fields. Each of these fields is optimally a strip at least several arc minutes long; the task group has chosen a region 2.5 arc min wide by 12 arc min long for the purpose of estimating the limiting magnitude. Since the InSb sensor has a nominal field of view of only 54 arc sec, many pointings will be needed, even for this relatively small strip. One pointing in each of these fields would also be chosen for an ultradeep survey (see next section).

The primary imaging band (with a nominal $30 \%$ bandpass) would be centered on the background minimum, as defined by the ATD/NTOT's temperature and the zodiacal background but likely to be near 2.5 microns. In such a band, the limiting magnitude at a signal-to-noise ratio of 5:1 can be expected to be approximately $\mathrm{K}=29$ in 10 hours of integration (for an unresolved source-and based on the system parameters listed in Chapter 2). Although galaxies will typically be resolved, there are indications from recent HST images that the source size will be small. Very faint sources appear to have half-light radii of $<0.5 \mathrm{arc} \mathrm{sec}$, and probably nearer $0.3 \mathrm{arc}$ sec for typical galaxies at $z=1.0 .3,4$ Thus the loss in limiting magnitude will not be large for distant galaxies (a reasonable estimate might be $\mathrm{K} \sim 27$ ). To cover the above strip with images would require 42 pointings, each of 10 hours, for three fields for a total of 1260 hours of integration.

While the primary waveband should be centered on the background minimum, observations of additional bands can broadly define the redshifts of the sample objects because of the dramatic changes in luminosity occurring across major spectral features (e.g., the $\mathrm{H} / \mathrm{K}$ break at 0.4 micron). Several additional bands, probably two on the blue side and one on the red side of the minimum, should be selected. These need not go as deep. If an optical framing camera is added to the ATD/NTOT's baseline instrument suite, simultaneous broad-band optical (nominally I-band at 0.8 micron) imaging would also be carried out on these fields. Simultaneous use of the framing camera would provide one of the blue bands at no incremental cost in observing time. Since this optical image would be obtained along with every infrared integration, the limiting magnitude would be large (but against a zodiacal background that is substantially larger than that at 2 to 4 microns). If 5 hours per pointing is allocated per band (for each of the two additional infrared bands), or an additional 10 hours per pointing, a further 1260 hours are needed to obtain the additional colors.

It is unlikely that accurate redshifts can be obtained for many of the distant galaxies that would be imaged with ATD/NTOT. Only those with magnitudes brighter than $K \sim 21$ are likely to be within the reach of 8- to 10-meterclass telescopes with high throughput spectrographs. Thus it is very important to estimate the redshifts from infrared colors as described.

\section{Ultradeep Survey}

What does the universe look like at the faintest possible levels? The ATD/NTOT could go to fainter magnitudes and look deeper into the universe than any other telescope has ever done. An ultradeep survey of a small region of the sky through a broad filter centered on the background minimum could be used to define the magnitude/number-count relation $\mathrm{N}(\mathrm{m})$ to unprecedented levels. The size-distribution/magnitude relation could also be determined, as could the surface density of objects as a function of magnitude. Existing HST data suggest that the sizes and shapes of high-redshift galaxies are dramatically different from those of objects at intermediate redshifts. 
One could also envisage carrying out a measurement of the cosmic background light, that is, the unresolved background flux. This would require careful modeling and subtraction of the resolved sources. Given the problems with confusion of foreground galaxies mentioned above, this undertaking will not be practical with conventional ground-based telescopes. Even equipping ground-based telescopes with adaptive optics will not help, since the project requires a very stable and quantifiable point-spread function that cannot be achieved given the variable seeing ground-based telescopes suffer. Furthermore the infrared array must be very stable and well calibrated.

Logistically, the most practical approach to this ultradeep survey would be to center the field in one of the strips used for the deep galaxy survey. Since the InSb fields are so small, it would be very prudent to have a single pointing in each of the fields, so as to evaluate the dispersion in the counts over several independent fields. Within the allocated budget a single integration of 100 hours per strip would be practical. This is 10 times longer than the other integrations discussed so far, thus satisfying the goal of a substantial increment in depth (for galaxies one might expect to get to $\mathrm{K} \sim 29-30$ !). Together with the strip survey, the ultradeep survey fills the nominal 2800 hours for the deep zodiacal minimum surveys. If additional time were available it might be valuable to obtain increased integrations in two other infrared filters at that pointing. Again, as noted above, the addition of an optical framing camera would allow I-band images to be obtained contemporaneously with each of the infrared images, thereby building up an ultradeep optical image.

These images would provide a means of establishing, albeit crudely, some idea of the redshift distribution of the detected objects. Unusually large color changes would indicate, as discussed above, that major spectral features such as the Lyman limit, Lyman Alpha, or the H/K break could lie between the filter bandpasses.

\section{Cosmological Tests}

Over the next few years it is expected that substantial progress will be made in establishing the geometry of the universe from survey observations of type-1 supernovae and the use of other "standard candles" at intermediate redshifts (e.g., using internal velocities for fundamental plane studies of ellipticals galaxies). However, as history has shown, such tests are likely to be less definitive than expected. Thus tests at higher redshifts will likely be needed to minimize the uncertainty in the determinations of the cosmological parameters. The sensitivity to different cosmologies is just too small at low redshifts to provide a highly accurate determination of the deceleration parameter, $q_{0}$. The relative values of $\Omega_{\text {baryon }}, \Omega_{\text {matter, }}$, and $\Omega_{\Lambda}$ are therefore likely to remain in dispute for some time to come. Supernovae or certain classes of objects may well provide useful probes of the scale of the universe at high redshifts.

The ATD/NTOT could help begin the exploration for suitable standard candles, even if it is unable to complete the program during its lifetime. It could be a pathfinder that would identify some of the central goals of cosmology that would be addressed by a subsequent, longer-lived, and more capable astronomy mission.

\section{KUIPER DISK SURVEY}

Since comets represent some of the least altered objects left over from the formation of the solar system, the study of comets provides important constraints on solar nebular models. However, to use these constraints, astronomers must understand where in the solar nebula these primitive objects formed and, therefore, where the constraints are pertinent.

Known comets are believed to have originated in one of two reservoirs in the solar system. The first of these is the Oort cloud, a spherical ensemble of comets that surround the Sun at distances of $10^{3}$ to $5 \times 10^{4} \mathrm{AU}$. The comets in the Oort cloud probably formed in the Uranus-Neptune zone and were ejected outward by planetary perturbations. Perturbations pumped up the inclinations until a halo of objects resulted. Comets from the Oort cloud are occasionally nudged into a random walk toward the inner solar system by the passage of nearby stars, interstellar clouds, or other disturbances.

However, more recently, it has been noted that the comets with the shortest orbital periods (less than 20 years), the so-called Jupiter-family comets (JFCs), all have inclinations that are near the plane of the ecliptic. Since no known mechanism could preferentially flatten the inclinations of these JFCs when they were perturbed from the 
Oort cloud, it has been argued that the source of the JFCs must be a disk of comets whose inner edge is just beyond the orbit of Neptune. ${ }^{5,6}$ This disk is now known as the Kuiper Disk because it was Gerard Kuiper ${ }^{7}$ who pointed out that unless there was more material past the orbit of Neptune, the solar system would have a sharp edge to its mass distribution, which was unlikely. These comets likely formed where they are currently located and have undergone little or no evolution.

Until recently, the Kuiper Disk was only a theoretical construct because there was no observational evidence for its existence. Since 1992, however, more than 20 objects with trans-neptunian orbits have been found. All have $\mathrm{V}$ magnitudes of 23 to 25 , putting them near the limits of ground-based detection. Assuming a typical cometary albedo of 0.04 , these objects all have radii from 50 to $100 \mathrm{~km}$, most at the larger end. These sizes are inconsistent with the sizes of known JFCs, which typically must have radii of 1 to $20 \mathrm{~km}$. Astronomers know of no mechanism for converting 100-km Kuiper Disk objects to 10-km-sized cometary nuclei.

Thus, our knowledge of the JFC parents is still not firm. It is critical to our understanding of conditions in the early solar nebula to provide a link between the Kuiper Disk and the JFCs. Finding the link requires finding Kuiper Disk objects whose sizes are more typical of those of JFCs. However, at $40 \mathrm{AU}$, the inner edge of the Kuiper Disk, a $10-\mathrm{km}$ object with an albedo of 0.04 would have a $\mathrm{V}$ magnitude of 28.5 , much too faint for detection by ground-based surveys.

While the HST with its current Wide-Field/Planetary Camera 2 (WFPC2) can reach such faint limiting magnitudes, it requires a combined integration time of 5 hours to reach signal/noise 4 for $V=28.5$. With the HST's low orbit and the resultant Earth occultation periods, it takes approximately 30 hours to obtain the necessary 5 hours of integration time. Even at quadrature, when Earth parallactic motion is zero, an object at $40 \mathrm{AU}$ would move 15 arc sec (or 150 pixels on the wide-field CCDs of the WFPC2) in 30 hours. Additionally, the WFPC2's field of view is small (around 4.2 square arc min for the three wide-field CCDs), and observing time is highly competitive. The combination of these factors means that although the HST can observe deeply enough to image objects of the size of a large comet at $40 \mathrm{AU}$, an extensive survey for such objects is not an appropriate use of the HST.

With a 4-meter space telescope, especially one with a Molniya orbit or another orbit that does not suffer from frequent Earth occultation, a definitive survey for the Kuiper Disk could be undertaken. As noted in the previous section, the larger aperture and higher resolution of the ATD/NTOT should allow researchers to observe point sources with $\mathrm{I}=29.5$ (V $\sim 30$ for typical colors) (i.e., objects with radii $R \sim 6 \mathrm{~km}$ at $r=40$ AU or with $R=100 \mathrm{~km}$ at $r=150 \mathrm{AU}$ ) in a 1 -hour integration. One could even detect a Pluto-sized object in the inner Oort cloud. Since the Molniya orbit allows multiple 1-hour exposures per orbit (probably five to eight) whereas the HST orbit requires 30 hours to complete a single 5 -hour integration, the difference in results is dramatic.

The ATD/NTOT would allow astronomers to observe $10-\mathrm{km}$ objects to $50 \mathrm{AU}$. Assuming the canonical $r^{-3}$ distribution of bodies with size, researchers should find roughly 1000 objects per square degree, or one in every few fields, even with the very small field of view of a single $2048 \times 2048$ chip.

It should be noted that the $2048 \times 2048 \mathrm{CCD}$ projected under the task group's proposed enhancements would have pixels of 0.03 arc sec or a field of view of only 1.1 square arc min. Therefore some of the ATD/NTOT's speed advantage over the HST is negated by the even smaller field size. However, a 5-hour integration could be achieved in a single orbit, making this telescope much more efficient than the HST for carrying out such a survey.

A very deep survey covering a significant region of the sky would enable a study of the mass distribution of the Kuiper Disk. Current ground-based surveys are restricted to the brightest and largest objects, and only a very small region of the sky can be examined by the HST. For the latter, distances and sizes must be computed based on assumptions about the orbit of candidate objects, since it will be impossible to follow up the original observations and compute orbits. With a more complete survey using the ATD/NTOT, it will be possible to compute actual orbits for objects if follow-up observations are planned.

\section{Properties of Kuiper Disk Objects}

The group of 20-plus known, large Kuiper Disk objects is a critical confirmation that trans-neptunian objects exist. However, their link with the JFCs is uncertain. A mass distribution is needed to constrain dynamical 
integrations of the lifetimes of such objects. A comparison between the observations of JFCs and the numerical integrations of Levison and Duncan ${ }^{8}$ or Holman and Wisdom ${ }^{9}$ shows that the current population of Kuiper Disk objects is smaller by a factor of 1000 to 10,000 than a simple extrapolation of the surface density in the outer planetary region would predict. This discrepancy leads to the conclusion that there is a dip in the surface density of the solar system outside the orbit of Neptune. Knowing the size distribution of Kuiper Disk objects can allow unique differentiation among three possible explanations for this feature:

1. There was an edge to the original solar nebula,

2. The Kuiper Disk initially had a much larger mass but evolved to its current mass due to collisions, or

3. The early migration of Neptune (either inward or outward) dynamically cleared the inner portion of the Kuiper Disk.

In order to continue progress in our understanding of the Kuiper Disk, it is not sufficient to continue to discover the objects that are the largest members of the class. Thus, there will come a limit to the effectiveness of ground-based surveys. The small field of view and the nature of the way in which the HST is scheduled do not allow for a detailed survey, although the HST could obtain observations of an interesting, small, limiting size. Indeed, the HST recently obtained suggestive, but as yet unconfirmed, evidence of the existence of a population of small Kuiper Disk objects. With the much longer continuous viewing time of the Molniya orbit, a 4-meter telescope that undertook as a project to complete the surveying of a large piece of sky (models predict $100010-\mathrm{km}$ objects in 1 square degree) to $I=29.5$ or $V \sim 30$ would finally achieve a definitive picture of the mass distribution in the Kuiper Disk (Figure 5.3), both according to the size of the object and, for objects like those already discovered, with distance from the Sun. Assuming 16 useful hours per day of observation in the Molniya orbit, such a survey would take roughly 8 months, a reasonable share of a 5-year mission.

\section{Follow-up and Related Projects}

Depending on the details of the operations of the telescope, a variety of more or less automatic follow-up programs could be executed on each object discovered in the Kuiper Disk. These observations would be aimed at determining the orbit quickly and determining some of the basic physical properties. The first such follow-up observation would be a sequence of observations from opposite sides of the spacecraft's orbit around Earth in order to directly determine the distance to the body (see Chapter 4). This would allow a rapid separation of Centaurs, Neptune librators, and transition objects from true Kuiper Disk objects.

Another important follow-up observation would be to obtain colors of the Kuiper Disks objects and to determine their photometric variability in order to understand the distribution of rotational periods of these objects and even to place constraints on their deviations from a spherical shape. Observations to date have shown that at least one of the known trans-neptunian objects exhibits significant variability in its brightness. All of these observations are straightforward with the ATD/NTOT once the object has been discovered and its motion determined to sufficient accuracy that it can be found again. The task group notes that an I-K color would be reliably determined automatically for objects in the central part of the field, since the InSb detector would presumably be taking data at all times also.

Closely related programs can also be carried out in a survey mode. One obvious choice is to image all known cometary nuclei as close as possible to their perihelia in order to estimate nuclear sizes (assuming an albedo), estimate nuclear rotational periods, and determine nuclear colors. Lamy and Toth ${ }^{10}$ have already demonstrated the great advantage of the HST over ground-based observations for photometrically separating the nucleus from the coma, even when comets are near the Sun and have significant comae.

The potentially superb spatial resolution of the ATD/NTOT at optical wavelengths enables higher spatial resolution than with the HST, and the large collecting area makes it feasible to carry out the observations when comets are further from the Sun and therefore when cometary activity is weaker. The task group notes that the point-spread function of the ATD/NTOT will have a full width at half maximum (FWHM) of roughly $150 \mathrm{~km}$ for a JFC near aphelion and about $300 \mathrm{~km}$ at Chiron. Although this performance is not sufficient to spatially resolve 


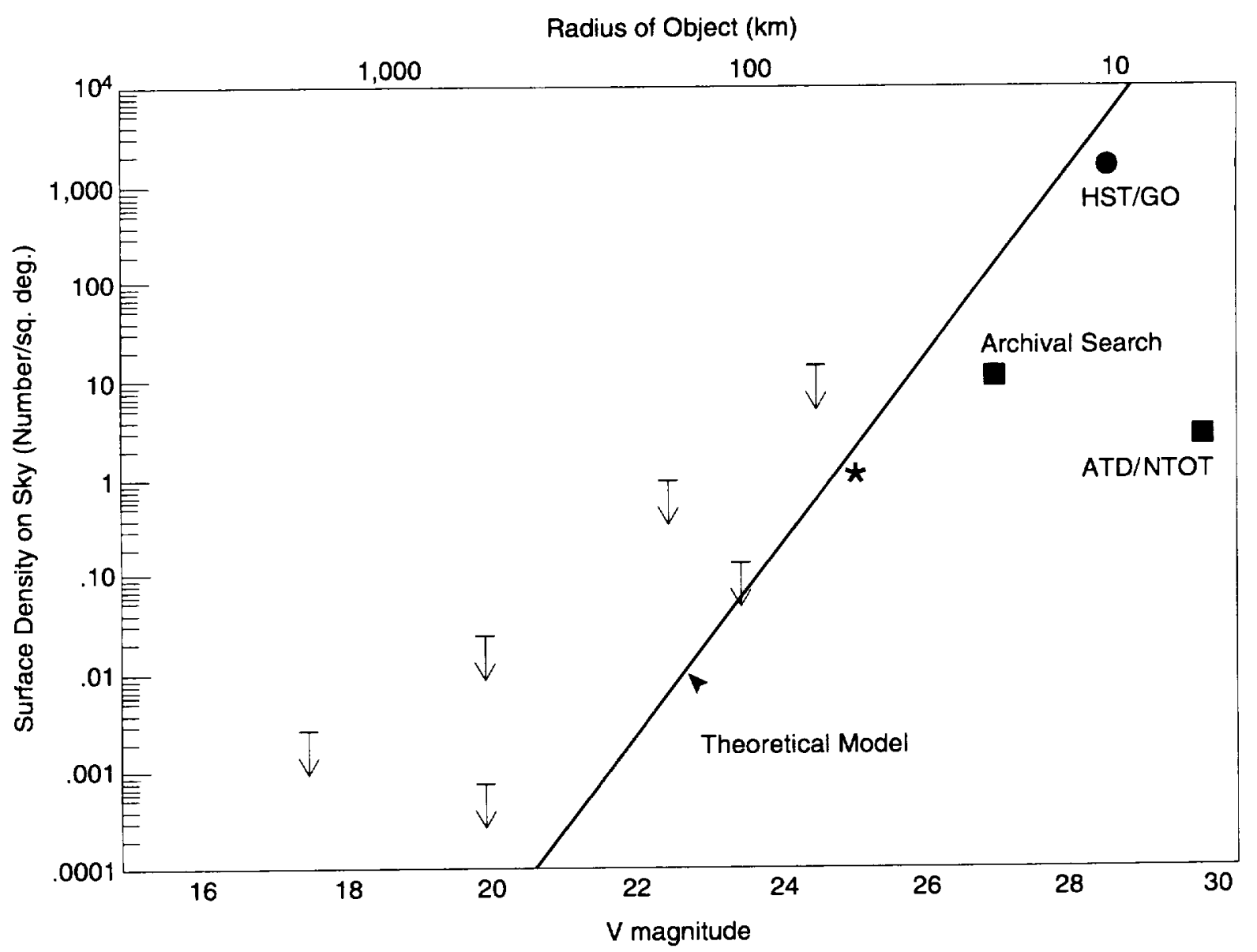

FIGURE 5.3 A comparison between various searches for Kuiper Disk objects and a numerical model of the size distribution of these trans-neptunian primitive bodies. The number of Kuiper Disk objects per square degree as seen on the sky brighter than a certain $V$ magnitude is shown as a function of that magnitude. The top axis shows the size of the smallest object at 40 AU detectable with these surveys (assuming an albedo of 0.04). The dashes with downward pointing arrows represent upper limits determined by unsuccessful surveys. The success of the model is indicated by noticing that all the unsuccessful searches resulted in points lying above the curve and that the survey (asterisk) that discovered the Kuiper Disk objects 1992 QB1 and 1993 FW lies below the curve. The model also predicts that the ongoing so-called HST/GO and Archival searches should be successful in detecting objects as small as 10 to $20 \mathrm{~km}$ across. Use of the ATD/NTOT would open a much larger region of search space for investigation. A 250 -day survey to $\mathrm{V}=30(\mathrm{I}=29.5)$ would cover 1 square degree and thus set three-sigma limits of three per square degree as shown.

the nucleus, it is sufficient to photometrically resolve, and thus determine the nuclear brightness from, any coma that is strong enough to matter.

Nuclear colors, particularly those extending into the near infrared, are extremely important for understanding the physical nature of cometary nuclei. Colors are particularly important for determining whether there is bare ice on the surface, either everywhere on the surface or in patches such as the active areas observed on Halley's comet.

The ATD/NTOT should be capable of measuring even the near-infrared colors for some comets at aphelion and for others when they are closer to the Sun. Observations at other points in their orbits would also be invaluable for understanding the onset of activity as periodic comets approach the Sun. Since the number of known shortperiod comets is less than 200 , this entire program is only a small part of the basic survey of the Kuiper Disk. 


\section{STAR- AND PLANET-FORMING REGIONS}

As noted above, the key research areas identified in the Bahcall report include the origins of stars and planets. ${ }^{11}$ The study of star-forming regions is another area that can benefit dramatically from an extensive survey with the ATD/NTOT if it performs as desired.

At the time of the ATD/NTOT's flight (presumably shortly before the turn of the century), adaptive optics will enable large, ground-based telescopes to often achieve high Strehl ratios in the infrared, although limitations on sky coverage will remain unless laser guide-star systems are routinely in use. Thus, the ATD/NTOT's resolution, as such, in the infrared is not a dramatic gain for addressing these problems. On the other hand, studies of starforming regions require extracting every possible bit of resolution from the telescope. Thus if its point-spread function (PSF) is very stable, the ATD/NTOT will have a major advantage over ground-based telescopes whose PSFs are certain to be rendered unstable by variable seeing. Furthermore, even at 2 microns, studies at NASA's Infrared Telescope Facility (IRTF) have shown that neither the Keck nor the Gemini telescopes will be able to compete with IRTF with tip-tilt (and thus with ATD/NTOT) until they adopt full, high-order adaptive optics.

The ATD/NTOT's much bigger advantage will occur at visible wavelengths because adaptive optics will not have been developed to a sufficient extent to allow multimeter-aperture, ground-based telescopes to achieve high Strehl ratios. Thus the PSF of the ATD/NTOT will provide a dramatic increase in resolution so long as the performance expected from an enhanced primary mirror (see Chapter 2) is achieved. This increase in resolution will be particularly advantageous if the PSF is stable over long periods.

Clearly an optical framing camera of astronomical quality will be required to take advantage of these technological gains in the telescope. Ideally, a coronagraph should also be installed to minimize the scattered starlight when looking for or at faint disks around stars. But it appears to this task group that the quality of the primary mirror and the significant diffraction by the secondary supports do not justify a coronagraph. Even without a coronagraph, however, the ATD/NTOT has the potential to make significant incremental advances in studies of star formation.

The following sections describe several important observing campaigns that would take advantage of the unique capability of an enhanced ATD/NTOT with a stable PSF to address three separate phases in the formation of stars and particularly planets.

\section{Embedded Young Stellar Objects}

The task group believes that the ATD/NTOT can address three of the goals for understanding the phase of star formation concerned with embedded young stellar objects. These include:

- Quantifying the frequency of disks in star-forming regions of differing densities,

- Inferring the structure of envelopes surrounding forming stars, and

- Determining the duration of the envelope-infall phase for stars of different mass.

\section{Frequency of Disks}

The first goal is to quantify the frequency of disks around stars of masses ranging from 0.1 to 5 solar masses in star-forming regions of differing stellar densities. The effect of stellar density on the formation of disks around protostars is an important open question that dramatically affects the inferred number of planets around other stars. The appropriate technique to investigate this question is to use imaging in the J, H, K, L, and M color bands of regions in which the density of young stellar objects (YSOs) is low (i.e., the mean separation between stars is $\sim 0.1$ parsec) and high (i.e., the mean separation between stars is $<0.01$ parsec). As noted in Chapter 3 , observations in the $\mathrm{L}$ and $\mathrm{M}$ bandpasses are far better done from space because of the dramatically reduced background emission. A space telescope's advantages relative to those of a warm, ground-based telescope enable sensitive imaging of deeply embedded YSOs, including those still shrouded by their natal cores. This imaging would allow extremely young, embedded stars $\left(A_{v}<50\right)$ to be examined and disk frequencies and lifetimes to be evaluated over the entire mass range. The ATD/NTOT's high angular resolution is also crucial because dense star clusters, which are 
forming the full range of stellar masses, are found only at distances greater than some 500 parsecs where the projected separations between objects in a dense cluster are only of the order of 1 arc sec.

\section{Protostellar Envelopes}

The second goal is to quantify the structure of envelopes surrounding forming stars. It should then be possible to infer the range of both angular momenta (characterizing protostellar cores) and centrifugal radii (characterizing the disks surrounding young stars) as a function of stellar mass. This characterization is best done by comparing models of infalling envelopes, computed for a range of initial conditions (e.g., core sound speed, mass, rotation), with JHKL imaging of the scattered-light envelopes surrounding embedded YSOs.

The study would also ideally utilize narrow-band (e.g., [Fe II] at 1.64 microns; $\mathrm{Br} \gamma ; \mathrm{Br} \alpha$; and $\mathrm{H}_{2}$ at 2.2 microns) imaging to locate and characterize jets, which would establish the rotation axis of the system and the interaction between stellar jets and infalling matter. The task group notes that the limited instrumentation in the focal plane might not allow a wide selection of filters. The filters themselves, however, would cost only a nominal amount if the capability were present to install and use them.

These observations would allow imaging of embedded stars out to the distance of Orion and would enable properties of the envelope and disk to be inferred from scattered-light patterns around stars of differing mass. The presence of disks and the magnitude of their centrifugal radii would be inferred from the scattered-light patterns. ${ }^{12}$ The presence of disks with centrifugal radii ranging from 20 to $200 \mathrm{AU}$ should be readily inferred due to the ATD/ NTOT's high angular resolution (FWHM $\sim 20$ AU for the near infrared at Orion).

\section{Envelope Infall and Other Projects}

The third goal is to determine empirically the duration of the envelope-infall phase around stars of different mass. As with the two previous projects, the appropriate technique is to use JHKL imaging to search for the scattered light patterns produced by dust in rich, embedded clusters. These images can be the same ones used for the second project.

Other interesting observations (e.g., those aimed at diagnosing the presence of accretion-driven jets and winds) would benefit from narrow-band images in the light of $\mathrm{H}_{2}$ lines. High-angular-resolution observations of jets associated with YSOs spanning a range of masses and relative evolutionary states would enable characterization of jet collimation and wind-envelope interactions, and provide important insight into the role played by winds and jets during the early stages of star formation. The advantages of the ATD/NTOT would be its high angular resolution and the ability to obtain deep-infrared images of heavily obscured YSOs in their earliest evolutionary phases.

\section{Accretion-Dominated Phase}

Even when a YSO's optically thick, infalling envelope disappears, it is still surrounded by a massive, optically thick accretion disk. There are two main goals for understanding this so-called accretion-dominated phase of star and planet formation:

- Determining the sizes of disks surrounding stars of differing mass, and

- Understanding wind and jet morphology and collimation as a function of evolutionary state.

\section{Disk Sizes}

Observing scattered-light patterns is a good way to determine the sizes of disks. This is best done with $\mathbf{R}$ - and I-band imaging of a sample of optically visible YSOs in nearby ( 150 parsecs) star-forming regions. Selecting which stars to examine is best done by looking for signatures of disks in their spectra. For geometrically flat disks, the disk/star contrast ratio may be less than 0.001 and thus extremely difficult to observe. The suitability of the ATD/NTOT for this project would need to be evaluated after the telescope has been thoroughly tested in orbit. 
These observations would also be influenced by the presence of scattered-light contributions from dust embedded within an optically thin, remnant infalling envelope. With the correct geometry, this contribution might overwhelm that from the disk. Detecting such envelopes is interesting for understanding star formation but not (directly) for understanding the formation of the solar system.

\section{Winds and Jets}

The second goal is to examine the wind and jet morphology and understand wind collimation as a function of evolutionary state. Optical (R, I) probes (e.g., [S II], which is formed and excited in shocks) would enable imaging on scales of $5 \mathrm{AU}$.

\section{Post-Accretion Phases}

After the accretion phase is over, YSOs have disks that are populated by "secondary" dust produced by collisions between planetesimals. The study of these YSOs, called weak T Tauri stars, represents potentially the most exciting arena for ATD/NTOT observations. Astronomers would first observe a sample of several hundred weak T Tauri stars in nearby star-forming regions and search for evidence of low-surface-brightness scatteredlight disks. Observations in the R and I bands would take full advantage of the ATD/NTOT's high angular resolution to image scattered-light disks and resolve objects as small as $5 \mathrm{AU}$. Disk sizes and radial surface densities could be inferred from surface-brightness distributions.

The presence of "dust-free" holes in disks might provide the strongest, although indirect, evidence for the presence of planets of sufficient mass to sweep up the material and create a void between the inner and outer disks. By targeting stars whose spectra (from ISO and ground-based telescopes) suggest large inner holes, astronomers could search for evidence of such $(r>5 \mathrm{AU})$ holes in a disk's surface-brightness distribution. Narrow-band filters could be used to attempt to carry out mineralogical studies of disk dust, at least for those mineral species containing $\mathrm{Fe}^{+}$and $\mathrm{Fe}^{2+}$ that have absorption features in the 0.5- to 5.0-micron spectral range. Because of the low surface brightness of some such disks, the capability to do this project must be tested on orbit when the ATD/ NTOT's actual PSF has been evaluated.

\section{GLOBAL CHANGE ON PLUTO AND TRITON}

Numerous synoptic problems relating to planetary atmospheres are well suited to the high spatial resolution of the ATD/NTOT, although the advantage of using the ATD/NTOT rather than the HST are not particularly clear. One particular project relating to Pluto and Triton stands out because it is more closely related to origins than the others that immediately come to mind and also represents a much more significant demonstration of technological capability.

Pluto and Triton are of interest because these bodies most likely formed at the same time as the Sun and not when the other planets formed. As such, they provide a sample of objects somewhat larger than those in the Kuiper Disk whose formation was contemporary with the Sun's. Bodies of this type may have been the building blocks of the outer planets. Their relatively large sizes mean that they are differentiated bodies and are massive enough to retain atmospheres, with surface pressures in the microbar range.

Because of its low gravity, Pluto's atmosphere is the largest in the solar system relative to the size of the body. As a consequence, Pluto's outer atmosphere is in hydrodynamic escape. Unlike the jovian planets, the major constituent of Triton's and Pluto's atmospheres, $\mathrm{N}_{2}$, is likely to be in vapor-pressure equilibrium with surface ice. These atmospheres also contain small amounts of $\mathrm{CH}_{4}$ and $\mathrm{CO}$. The only other body in the solar system whose atmosphere is known to be in vapor-pressure equilibrium with surface ice is Mars, whose principal atmospheric constituent is $\mathrm{CO}_{2}$.

Pressure changes of several orders of magnitude have been predicted to occur as Pluto and Triton respond to seasonal changes in insolation. This behavior arises because, at the temperatures involved $(\sim 40 \mathrm{~K})$, vapor pressures are extremely sensitive to temperature. The magnitude of these surface-pressure changes depends on the sublimation-condensation properties and extent of subsurface volatile reservoirs. 
Learning about how the atmospheric structure of these bodies changes over time scales of months and years is necessary for understanding the complex relationships involved in surface-atmosphere volatile exchange, as well as the processes that control their basic atmospheric structure. For example, one model predicts that the structure of Pluto's middle and lower atmospheres is controlled by $\mathrm{CH}_{4}$, whereas another predicts that $\mathrm{CO}$ is more important. The more we learn about these atmospheres from remote observations, the more effectively we can plan investigations to be conducted by future spacecraft missions to these bodies.

The structure of Pluto's atmosphere has been probed at only one time--by a stellar occultation in 1988. The structure of Triton's atmosphere has been probed twice-during Voyager 2's encounter in 1989 and by a stellar occultation in 1993.

If the technology demonstration of the ATD/NTOT's maneuverability (see Chapter 4) proves successful, then it would be possible to carry out a program of 1 to 2 atmospheric probes of these bodies per year, based on their (present) average rate of stellar occultations. This rate could be higher or lower, depending on the orbit of the telescope and the maneuverability demonstrated (i.e., how much, how accurately, and how often) during the testing phase of the ATD/NTOT mission.

The occultation rate is proportional to the fraction of time during an orbit when Triton or Pluto is visible. Also there would be a gain in the number of potential occultations that is proportional to the diameter of the ATD/ NTOT's orbit (projected onto the Earth's polar axis) relative to the diameter of the Earth. For a Molniya orbit this factor is 2.6 .

The ability to undertake this observing program does not depend on any of the enhancements suggested in Chapter 2. Rather, the decision should depend on the availability of resources to pay for the significant extra operational costs entailed in maneuvering the spacecraft to a new orbit or to a different position in its current orbit. It is also unclear to the task group whether or not the spacecraft would be usable for other astronomical tasks while the maneuvering was taking place. If not, the observational efficiency, defined as time collecting scientific data divided by elapsed time, would be extremely low. Low efficiency is not inherently bad, but it represents a value judgment that cannot be made until more is known about the maneuverability of the spacecraft.

\section{REFERENCES}

1. Astronomy and Astrophysics Survey Committee, National Research Council, The Decade of Discovery in Astronomy and Astrophysics, National Academy Press, Washington, D.C., 1991.

2. Space Studies Board, National Research Council, An Integrated Strategy for the Planetary Sciences: 1995-2010. National Academy of Sciences, Washington, D.C., 1995.

3. Phillips, A.C., Gronwall, C., Koo, D.C., Forbes, D.A., Illingworth, G.D., and Huchra, J.P., Astrophysical Journal Letters, 1995, submitted.

4. Driver, S.P., Windhorst, R.A., Ostrander, E.J., Keel, W.C., Griffiths, R.E., and Ratnatunga, K.U., Astrophysical Journal Letters, 1995 , submitted.

5. Fernandez, J.A., Monthly Notices of the Royal Astronomical Society 192:481, 1980.

6. Duncan, M., Quinn, T., and Tremaine, S., Astrophysical Journal Letters 328:69, 1988.

7. Kuiper, G., Astrophysics: A Topical Symposium, J. Hynek, ed., McGraw-Hill, New York, 1951, p. 357.

8. Levison, H.F., and Duncan, M.J., Astronomical Journal 100:1669, 1990.

9. Holman, M.J., and Wisdom, J., Astronomical Journal 105:1987, 1993.

10. Lamy, P.L., and Toth, I., "Direct Detection of a Cometary Nucleus with the Hubble Space Telescope," Astronomy and Astrophysics, 293L:43, 1995.

11. Astronomy and Astrophysics Survey Committee, National Research Council, The Decade of Discovery in Astronomy and Astrophys. ics, National Academy Press, Washington, D.C., 1991.

12. Kenyon, S.J., Calvert, N., and Hartmann, L., "The Embedded Young Stars in the Taurus-Auriga Molecular Cloud I: Models for Spectral Energy Distribution," Astrophysical Journal 414:676, 1993. 


\section{6 \\ Issues and Concerns}

The task group identified several areas of concern regarding the astronomical utility of the ATD/NTOT, some arising from the fundamental differences between the needs of space astronomy and the requirements of the experiments for which the telescope was designed, and others that are more generic and are based on experience with other missions. The concerns based on special astronomical needs include requirements for:

- Stabilizing images using faint guide stars;

- Minimizing background due to stray and scattered light in the optics and in the infrared by passive cooling;

- Understanding the impact of refiguring the mirror on observing efficiency; and

- Performing long integrations in a high-radiation environment;

The more generic concerns include the issues of:

- Analyzing the ATD/NTOT as a complete system;

- Ensuring the reliability of the software; and

- Controlling cost and schedule.

In subsequent sections the task group discusses at length aspects of these important issues that require further study during the final definition of the ATD/NTOT mission. The discussions are arranged roughly in a priority order reflecting the task group's interests and expertise.

\section{NEED FOR FULL SYSTEMS ANALYSIS}

In the course of its briefings from BMDO, Lockheed, Itek, and Charles Stark Draper Laboratories, the task group identified areas where there are clearly open or, as yet, undefined issues that could significantly affect the ATD/NTOT's performance of astronomical observations or valid demonstration of technology for future scientific applications. At present the ATD/NTOT is a collection of components that exist in one form or another in various parts of the relevant industries. However, little systems analysis has been done to validate the concept that the various components can be combined into a telescope that is useful for astronomical observations. The task group has no reason to think that they cannot be so combined, but a more complete systems analysis will be needed before launch. 


\section{IMAGE STABILIZATION AND GUIDE-STAR AVAILABILITY}

Astronomical operations differ markedly from the proposed DOD experiments in the need for stellar guidance input. It is worth noting that although the ATD/NTOT's collecting area is several times larger than the HST's, it has only about $1 \%$ of the available guidance field of view. Consequently, the ATD/NTOT must be able to produce a fine-error signal using very faint stars. The fine-guidance system as designed requires one guide star for controlling pitch and yaw and a second for stabilizing roll. The guidance analysis in the Lockheed/Itek briefing concluded that at 19th magnitude there is sufficient guide star availability and that the ATD/NTOT will achieve sufficient signal-to-noise to determine the centroid of a star's image to 0.003 arc sec, assuming a detector temperature of $260 \mathrm{~K}$ with a $10-\mathrm{Hz}$ sampling rate. The reliability of the estimates of signal-to-noise for centroiding was not clear to the task group. More importantly, however, guide stars will be significantly less abundant than assumed, especially at higher galactic latitudes.

The source of the average stellar density data presented in the Lockheed/Itek briefing is not known to the task group, but the briefing assumed an average guide-star density of about 25 per square milliradian at a limiting magnitude of 19. The task group's calculations, based on the Bahcall-Soneira model (Figure 6.1), show that for latitudes greater than 40 degrees, the density falls below this number, even when looking toward the galactic center. The situation is considerably worse when looking away from the galactic center, with densities almost five times lower. The stellar density remains low over the entire hemisphere facing away from the center of our galaxy and rises by less than 50\% in going from longitudes of 180 degrees to 90 degrees. Since the original estimate assumed success in finding guide stars only $80 \%$ of the time, it follows from Figure 6.1 that the success rate will be closer to $15 \%$ over most of the galaxy.

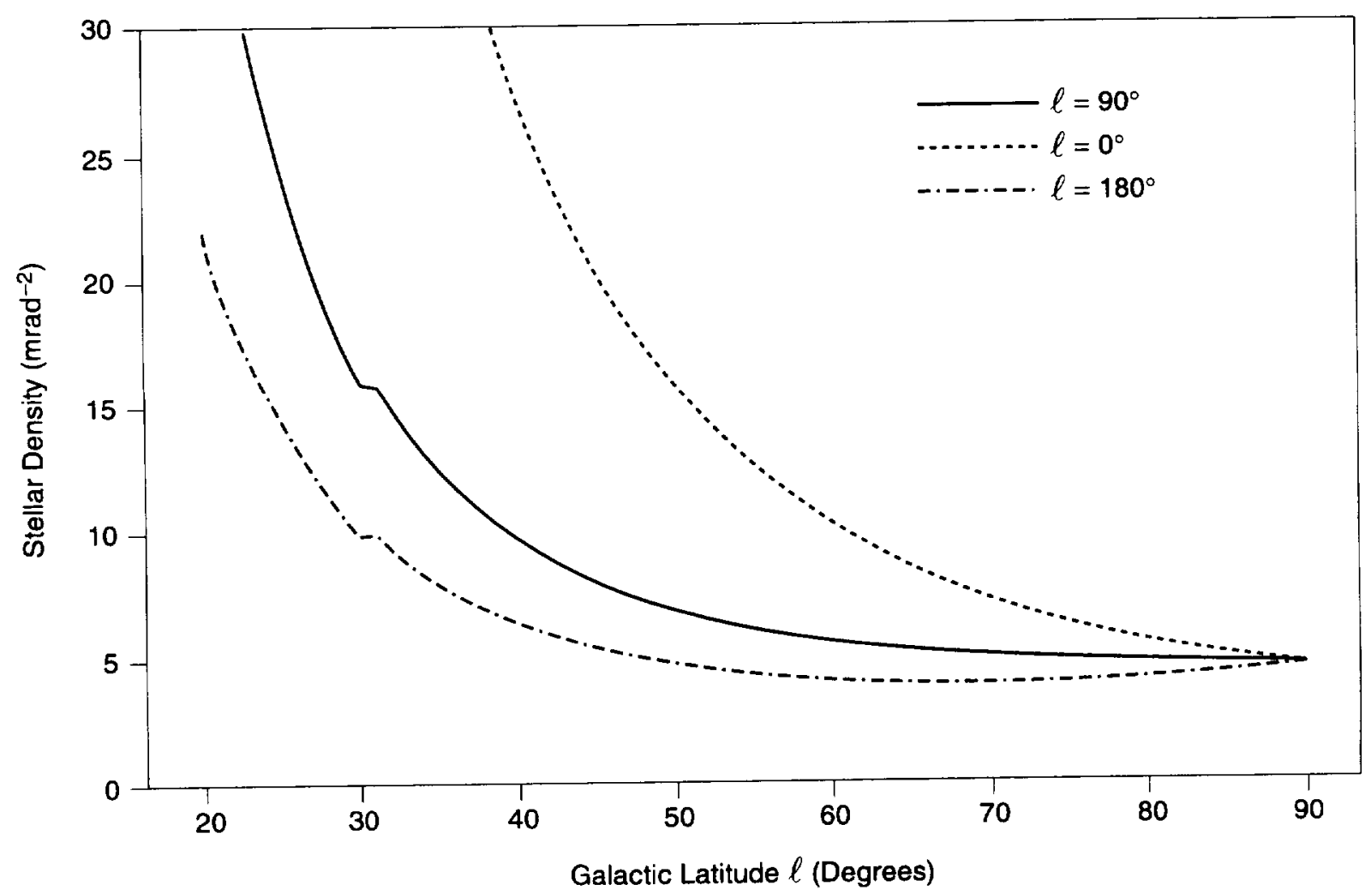

FIGURE 6.1 The number density of stars brighter than 19th magnitude, in the Bahcall-Soneira model of the galaxy, is shown as a function of galactic latitude for three different galactic longitudes. 
For many of the surveys considered as prime possible uses of the ATD/NTOT, the sky will be only sparsely sampled and the fields can be chosen so as to include suitable guide stars. For observations requiring specific fields, the lack of available guide stars can be accommodated by requiring only a single guide star for controlling pitch and yaw, while using an external star tracker for controlling roll. Clearly these issues must be explored in greater depth.

\section{STRAY AND SCATTERED LIGHT}

The issue of telescope background is another area where astronomical operations are likely to differ significantly from DOD's technological demonstrations. There is clearly a need in astronomy for a dark (i.e., skylimited) background. The ultimate utility of a large aperture is the ability to concentrate a great deal of energy in a small region of the focal plane, limiting the background contribution. As the background is elevated, faint objects are detected at lower confidence levels and, thus, require longer integration times. In other words, the background reduces the effective collecting area and limiting magnitude of the telescope.

Concerns about background arise from two design aspects of the ATD/NTOT. The first is the lack of a significant forward baffle. This deficiency increases the solar and lunar avoidance angles and allows more of the sky to contribute to stray light in the telescope. Stray light is a major issue at both optical and infrared wavelengths. The second issue is the large, rectangular cross section, tripodal truss supporting the secondary mirror. These elements provide flat specular surfaces facing the primary obscuration that permit photons to enter the focal plane area by direct reflection or by other paths through the optics.

Because the dynamic range of brightness is so large between the Sun and Moon, on the one hand, and deepspace objects, on the other, scattered light can be a tremendous problem for any studies of faint objects, that is, for the prime astronomical targets of the ATD/NTOT. Like the problem of a low density of guide stars, the problem of scattered light from specific, bright sources can be minimized by a careful choice of the fields of a survey. Avoiding bright objects is not, however, a general solution for all fields or for all types of scattered light. Further analysis of the scattered light is essential, and options should be explored for installing baffles and surface treatments to minimize specular reflections.

\section{FREQUENCY OF REFIGURING}

The ATD/NTOT derives much of its cost and weight leverage from its ability to fly a light, meniscus mirror with many actuators that can be reconfigured on orbit to compensate for low-spatial-frequency errors of figure. The input data for the optical correction is intended to come from an on-board, wavefront sensor. Although this sensor has been identified as slope measuring (and having a mass of $35 \mathrm{~kg}$ ), no information was available about its speed, dynamic range, noise, and calibration levels - all factors with a significant impact on astronomy operations.

It is envisioned that the influence functions of each of the primary mirror's actuators will be determined after launch. In all likelihood for astronomical operations, as with the HST, phase-retrieval analysis of images will provide better input to wavefront correction than the input provided by the on-board sensor. Thus it is reasonably possible that for astronomy operations, both in manipulating the influence functions and in sensing the wavefront, the reconfiguring of the optical system will not be autonomous.

Over the course of the acquisition, tracking, and pointing experiments for which the ATD/NTOT has been designed, it is unlikely that wavefront stability will be an issue. The experiments are of short duration and impose only modest limits on stability. Astronomical observations, however, characteristically require longer on-target times and therefore more demanding requirements on the telescope that arise in many different ways. At the very least, the requirement to reconfigure the primary mirror together with the possible need for interaction with the ground is a time overhead factor on operations. The Molniya orbit envisioned for the astronomy phase of the operation involves a dramatically varying thermal environment whose effect on the telescope point response function has not been quantified.

The undefined character of thermal and other figure drivers means that their impact on the required rate of figure correction is not known. Moreover, between corrections, the telescope point-spread function will degrade 
from a just-corrected to a needs-to-be-corrected state. The amplitude of this variation can be controlled at the expense of observing overhead as long as the intercorrection period is long compared to a typical integration time. Should the required correction period become too short, then the nature of the science that can be accomplished will be seriously compromised.

One option for exercising a more deterministic control of image quality is to close a slow feedback control loop around the figure actuators driven by output from the wavefront sensors. This option assumes that autonomous figure correction is adequate for astronomy operations. In effect, the figure control loop becomes an analog of the pointing control loop. Assuming a low signal-processing overhead, the speed of the loop is driven by the rate of photon arrival into the wavefront sensor. Assuming that a 19 th-magnitude star is used for figure sensing, Lockheed and Itek have estimated that the figure could be updated at intervals of a few minutes. The viability of this approach will depend on the quality of the correction data supplied by the wavefront sensor, the ability of the system to correct the wavefront without disturbing the field, and the availability of a suitable star for a phase reference. In light of the guide-star problem discussed above, this issue needs careful study. Since this issue is inherent to the ATD/NTOT's technology, perhaps the optimum approach is to implement the necessary control software and investigate it during the technology-evaluation phase of the mission.

\section{SOFTWARE RELIABILITY}

Since the Clementine project was carried out under constrained schedule and cost, the lessons learned from it can be applied to the proposed ATD/NTOT. For a variety of reasons, software development has proven more difficult to plan and complete on schedule than has hardware development. The software to run the Clementine mission had not been completed by the time of launch and was still being written during the early stages of the mission. Lessons learned from Clementine about software development include the following: ${ }^{1}$

- Take care to understand the impact of the software development environment on test and schedule;

- Realize that "non-commercial" processors are not supported as well as commercial ones;

- Assure that undefined processor states cannot enable critical circuitry; and

- Make maximum use of software verification tools prior to new uploads to the spacecraft.

\section{IMAGING DETECTORS IN A HIGH-RADIATION ENVIRONMENT}

A problem inherent in imaging arrays is their susceptibility to radiation events, often called cosmic rays. These events leave behind an enhanced signal that can span one or more pixels. Two factors contribute to the severity of these events. That is, the number of detected events is proportional to:

- The thickness of the imaging chip, with thicker chips trapping many more events and having longer detection paths; and

- The intensity of the radiation field.

Thus, thin, backside illuminated CCDs (such as the Texas Instrument chips in the HST's old WFPC) have fewer cosmic-ray events detected per unit time than thick, front-side illuminated chips (such as the Loral-Fairchild chips in the HST's new WFPC2). Similarly, detectors on ground-based telescopes are protected by the Earth's magnetic field, while the HST sees the enhanced radiation field in low Earth orbit. A spacecraft in a geostationary or Molniya orbit would spend much of its time above the protective Van Allen Belts. Thus, its CCDs would have a substantially higher event rate than corresponding chips in a ground-based telescope or the HST.

Experience with the HST has shown that cosmic-ray events can render less useful a significant fraction of the pixels on a chip. They also necessitate breaking total exposure time up into many 20 -minute subintegrations in order to be able to statistically eliminate cosmic-ray detections from the final images. There is a cost associated with such subintegrations, since reading out a CCD chip can take 1 to 2 minutes (though new low-noise output amplifier designs already suggest that much shorter readout times will become the norm)-time during which observations cannot be obtained. Additionally, for studies of faint moving objects, such as a Kuiper Disk survey, 
removal of cosmic-ray events can be problematic since the target object is moving relative to the background stars and can thus be mistaken for spurious events by many cosmic-ray detection algorithms.

As mentioned in Chapter 4, exploiting the ATD/NTOT's eccentric orbit to obtain very long exposures is an important technology demonstration of this mission. The feasibility of this test will depend on additional study of the extent to which cosmic-ray events can be handled, either by reducing them through shielding of the chip (although at least one side cannot ever be shielded), by finding intelligent ways to analyze data contaminated with such events, or by implementing continuing improvements in CCD technology.

\section{COST AND SCHEDULE CONTROL}

The planned ATD/NTOT mission is defined as a build-to-cost mission and not a build-to-specification mission. This constraint has important implications. If such a plan is continued, great care must be taken to ensure that the mission schedule does not slip, since time slips always result in higher costs and, if the costs are capped, a loss of capability. However, it is important that early in the mission, the capabilities be well defined so that all of the necessary pieces can be completed. A plan should also be assembled that dictates which specifications are most important in the event of a schedule slip, thus providing a mission with the most value for the budget.

Some of the astronomical projects discussed in this report can be accomplished with the baseline telescope and instruments. However, some important scientific projects and technology demonstrations will require enhancements, for example, an optical framing camera. Great care must be taken in deciding to add such instruments, since their addition has implications for the mission's cost and schedule. In today's cost-constrained NASA, it is not reasonable to perturb the budget greatly in order to add an instrument of the cost of the WFPC2. NASA and/or DOD should investigate alternatives, such as a university instrument of lower than class A or an instrument procured in the mode of Clementine or an instrument derived very directly from a camera currently being developed. It is also important that NASA and the astronomical community understand the short time scale needed for the delivery of such instruments and that the reasonableness of achieving timely production of such an instrument be considered.

If scientific investigations are to be an important part of the ATD/NTOT mission, then scientists will need to be included early so that appropriate decisions can be made regarding details such as filters. Such early inclusion means that the selection process for scientists will have to come early in the mission and be expedited.

Traditionally in NASA programs, the interval is long from formulation of a Research Announcement to the point when the personnel start work. The short development schedule anticipated for the ATD/NTOT does not allow a lengthy selection procedure if the goal of including scientists early is to be met. Similarly, the finite lifetime of the mission requires that personnel be used efficiently throughout the mission. Thus, consensus will have to be reached on how to achieve early involvement of scientists, in order to minimize any impacts on the delivery schedule and maximize the flexibility of the team to react to problems. Since such an approach is consistent with many of the goals being espoused within NASA today, the ATD/NTOT mission would provide an excellent opportunity to evaluate this new paradigm.

\section{REFERENCE}

1. Space Studies Board, National Research Council, Lessons Learned from the Clementine Mission, National Academy Press, Washington, D.C., in preparation. 


\section{7 \\ Conclusions and Recommendations}

The ATD/NTOT mission uses advanced technology that has important potential applications for future space astronomy missions. Furthermore, its advertised cost-effectiveness is crucial to NASA's ability to carry out significant space astronomy missions in an era of tightly constrained budgets. Both of these factors have not escaped the notice of other groups. Thus, both the High-Z and Polar Stratospheric Telescope concepts draw heavily on the capabilities of the ATD/NTOT's technology. This task group's basic conclusion is that the ATD/ NTOT mission does have the potential for contributing in a major way to astronomical goals. This is true both in the area of evaluating the ATD/NTOT's new technology for subsequent use in space astronomy missions, and in the area of performing selected, significant astronomical observations. There is a very high probability that the ATD/NTOT will be able to carry out astronomical programs that cannot be done with any other facility in existence or in development. Thus the task group's first and foremost recommendation is as follows:

1. To optimize the return to astronomy from the ATD/NTOT, the astronomical community should be directly involved in the continued study and development of this mission, including system engineering and complete mission analysis. These community representatives should be selected by NASA, and their role should be to advise NASA on the continuing value of this mission for astronomy. The group should include not only astronomers proposing specific observing programs, but also individuals with particular expertise in the design of large telescopes and space missions.

The task group's basic philosophy is that the ATD/NTOT mission is primarily a demonstration of technology for future national security missions, with a nominal lifetime of only 1 year. Furthermore, the mission is designed to cost, using existing technology, and it is not responsive to specific astronomical requirements. The astronomical applications are a bonus, a point of view that NASA and the astronomical community must share. Consequently, the task group recommends the following:

2. If the ATD/NTOT mission flies, a suite of tests of the suitability of its technology for astronomical applications - tests that may be different from the tests and demonstrations requested by DOD-should be carried out. Some of these tests can be conducted concurrently with the tests of defense technology, but others require an astronomical phase of operation after the conclusion of DOD's mission.

3. Although scientific goals must be kept in mind and accommodated insofar as possible during the planning of the ATD/NTOT mission, these goals should not impose requirements that would have a major impact on 
development or operations costs. In this sense the ATD/NTOT will be like possible future missions under the aegis of NASA's proposed New Millennium program.

4. The ATD/NTOT's astronomical promise is sufficient, even at this preliminary stage, that it is appropriate to plan for a mission phase devoted to astronomical observations. The resources devoted to planning an astronomical mission should be kept to a minimum until such time as the ATD/NTOT's scientific and technological capabilities are better defined.

If an extended mission occurs, the task group offers several additional recommendations regarding the way in which it should be managed. These recommendations reflect the philosophy that development costs for astronomical research programs should be kept to an absolute minimum because of the ATD/NTOT mission's nature as a demonstration.

5. An extended ATD/NTOT mission devoted to astronomical research should be carried out by a principal investigator and a science team, with rotating membership to involve people with different types of expertise during different phases of the mission. No provision should be made for a traditional guest observer program.

6. An extended mission should concentrate on extensive surveys that repeatedly use the ATD/NTOT in a single mode. Pointed observations of specific targets suggested by guest observers should be carried out only if they can be done simply by adding fields to the survey program and provided that suitable guide stars are available in those fields.

7. All scientific data collected during the ATD/NTOT mission should be delivered promptly to an existing public archive that is independent of and expected to outlive the mission. Such an archive might be that for the Hubble Space Telescope, the Planetary Data System, or the Infrared Processing and Analysis Center, with a specific choice to be made at a later date.

Because DOD sponsorship of the ATD/NTOT is uncertain, the mission's exact specifications are not yet clear. As noted in this report, the task group has assumed a baseline performance predicated on the requirements necessary to perform a mission that BMDO has now deferred. Several of the potential enhancements outlined by the task group would have a significant impact on the ATD/NTOT's astronomical capability relative to that baseline. Some of these enhancements may ultimately be required by, or at least be consistent with, a DOD mission if and when it is finally defined. All of the suggested improvements must be evaluated for their costeffectiveness during the system engineering phases of the mission. The task group has discussed its suggested enhancements with representatives of Lockheed and Itek. In the case of improvements to the figure of the primary mirror, the costs are well defined and the performance benefits reasonably well determined. In other cases, neither the costs nor the actual improvement in performance are very well determined, but it is the sense of this task group that the enhancements are likely to be very cost- effective and important for the astronomical aspects of the mission. In particular:

8. The figure of the ATD/NTOT's primary mirror should be improved by roughly a factor two to reduce its surface error to $\sim 17 \mathrm{~nm}$, and, thus, the total system's wavefront error to roughly $50 \mathrm{~nm}$ rms. This enhancement would allow both a better evaluation of the ATD/NTOT's technology and significantly enhance its astronomical potential. The cost of this improvement is reasonably well determined at $\$ 100,000$ according to representatives of Itek.

9. A large-format, framing, optical CCD of astronomical quality should be included in the ATD/NTOT's focal-plane package. This CCD should be optimized for performance in the far red (say, 0.8 micron) and should be of normal astronomical quality with respect to dark current, readout noise, and other relevant parameters. Its inclusion would enhance astronomers' ability to evaluate the technology and would lead to dramatic increases in the astronomical return from the mission. A less expensive but clearly less desirable option would be to replace the baseline line-transfer CCDs in the fine-tracking sensors with frame-transfer CCDs.

10. The ATD/NTOT's baseline InSb infrared array should be optimized for sensitivity by, for example, further cooling of the array itself and by minimizing the number of emitting surfaces in the optical path. The astronomical return would be significantly enhanced if the system ultimately reaches the sensitivity limit set by the zodiacal light. 
Some additional enhancements have been considered by the task group but were not discussed extensively with representatives from Lockheed and Itek. Furthermore, no quantitative details of these enhancements have been considered by the task group itself. It is likely that these enhancements would have a much greater cost impact on the mission than those discussed above. In addition, it is not clear if their implementation would be consistent with the goals of a possible national security mission. Therefore they are included here only for completeness and as suggestions for more detailed consideration at a later date. These improvements include:

- Modifying the ATD/NTOT's orbit to minimize and/or stabilize the thermal load on the spacecraft. The thermal load in the Molniya orbit varies significantly with orbital phase. Several possible alternatives exist, and their practicality should be explored.

- Optimizing the design to enhance the passive-cooling characteristics of the telescope and focal-plane instruments. Cooling could have a significant impact on the astronomical return and could enhance understanding of the technological aspects of, for example, stabilizing an active mirror at low and variable temperatures.

- Possibly adding dedicated instruments for scientific research either instead of or in addition to modifying the ATD/NTOT's instrument suite. Such an instrument might be an infrared camera with far fewer optical components in the path than are in the baseline design.

Even without all the possible enhancements, it is clear that the ATD/NTOT mission, at least if flown with the task group's suggested, critical enhancements (improved figure of the primary mirror, optical framing camera, and thermal control), can contribute dramatically to astronomy, both in the development of future missions and in the return of key astronomical data. The ATD/NTOT's advertised ability to make these contributions at relatively low cost, by using off-the-shelf components of previously unavailable technology, offers the possibility of dramatic breakthroughs for the future of astronomical missions in space. 


\section{APPENDIX}

\section{Glossary}

$\mathrm{AC}$

Active optics

Adaptive optics

Afocal

ALOT
Advanced Camera: a generic name for a number of different proposals to develop a third-generation camera to replace one of the Hubble Space Telescope's first or secondgeneration instruments in the late 1990s. In 1995, NASA selected the proposal called the Hubble Advanced Camera for Exploration.

A technique to take account of slowly varying forces, such as gravitational deflections and temperature drifts, that can distort a mirror on time scales of minutes to hours, resulting in imperfect images. This is usually implemented by means of a lookup table that provides values for actuator signals as a function of temperature and telescope orientation. These tables are initially generated by making observations of wavefronts from a distant source at a variety of temperatures and orientations.

A set of techniques to adjust the shape of mirrors on time scales of a fraction of a second to correct for rapid fluctuations in image quality. In the astronomical community, this term is used for systems that correct for distortions in images of ground-based telescopes due to atmospheric turbulence ("seeing"). In the DOD community there can be other local sources of distortion, such as in laser weapons systems, and the term is used to cover a wider range of applications. In both communities, the essential feature of the implementation is use of real-time sensing of the wavefront from a distant source to provide the signals to the actuators that control the shape of the mirror.

An optical system that receives parallel rays of light from a distant object and outputs parallel rays of light at a different magnification

Adaptive Large-Optics Technologies: a DOD-sponsored project at Itek Optical Systems that developed a complete, lightweight, 4-meter space telescope equipped with an advanced adaptive-optics system 
Anastigmat

Aperture

Aphelion

Apogee

Arc minute

Arc second

Array

Asteroid

ATD

AU

BMDO

Byte

CCD
An optical system that does not suffer from common optical defects such as coma, astigmatism, or spherical aberration

The diameter of the primary lens or mirror of a telescope; hence, the simplest single measure of the light-gathering power of a telescope

The point at which a body in orbit around the Sun reaches its farthest distance from the Sun

The point at which a body in orbit around Earth reaches its farthest distance from Earth

A unit of angle corresponding to $1 / 60^{\text {th }}$ of a degree. The full moon is 30 arc minutes in diameter.

A unit of angle corresponding to $1 / 3600^{\text {th }}$ of a degree; $1 / 60^{\text {th }}$ of an arc minute. An arc second is approximately the size of a penny viewed from about 2.5 miles.

Astronomical instruments that have recently been fabricated using new electronic components called detector arrays that consist of thousands of individual detectors constructed on centimeter-sized wafers of silicon, or other materials

An object orbiting the Sun that is smaller than a major planet (sub-kilometer to about $1,000 \mathrm{~km}$ in diameter) but shows no evidence of an atmosphere or other types of activity associated with comets. Most asteroids are located in a belt between Mars and Jupiter ranging from 2.2 to $3.3 \mathrm{AU}$ from the Sun.

Advanced Technology Demonstrator

Astronomical unit: the mean distance between Earth and the Sun, about 150 million $\mathrm{km}$ or 93 million miles

Ballistic Missile Defense Organization: the arm of the U.S. Department of Defense charged with developing missile-defense systems

A unit of information used in reference to computers and quantities of data. A byte consists of 8 bits ( $0 \mathrm{~s}$ and $1 \mathrm{~s}$ ) and may correspond to a single character or number.

Charge-coupled device: an electronic chip that has in recent decades replaced photographic emulsions as the primary recording medium for astronomical images. The recording portion of the chip is divided into discrete photosensitive elements (pixels) arranged in a rectangular array of rows and columns. The HST's CCDs have 640,000 pixels arranged in 800 rows and 800 columns. For various applications, some pixels are masked. An astronomical CCD has no masked area. At the end of an exposure, a shutter is closed to keep more light from coming in while the array is being read. The entire array of pixels is shifted, one column at a time, into a so-called column register at the edge, and after each shift the column register is read out slowly, row by row, through amplifiers and other circuitry. 
Clementine

Coma

Comet

Cosmic ray

Deceleration parameter

Diffraction limit

Discovery

DOD

Ecliptic

ESA

Figure

FOV

Frame-transfer CCD

F-SAT

FSM
A small-spacecraft technology demonstration mission launched by the DOD's BMDO in January 1994. The mission mapped the entire Moon but failed before flying by asteroid 1620 Geographos.

The spherical envelope of gas and dust surrounding the nucleus of an active comet

A volatile-rich body that develops a transient atmosphere as it orbits the Sun Observed comets usually have highly elliptical orbits, many approaching parabolic, although many comets in the Kuiper Disk are likely to have nearly circular orbits, as are many of the currently unobservable comets in the Oort cloud. When a comet comes near the Sun, some of its material vaporizes, forming a large head of tenuous gas, and often a tail.

A charged, subatomic particle of matter (not radiation), usually an atom stripped of all its orbiting electrons and accelerated to extremely high energies by unknown processes in space

A measure of the rate at which universal expansion is retarded by gravity

The finest detail that can be discerned with a telescope. The physical principle of diffraction limits this to a value proportional to the wavelength of the light observed divided by the diameter of the telescope.

A relatively new program within NASA's Solar System Exploration Division to fly frequent, relatively cheap missions, primarily in the inner solar system. NEAR (Near Earth Asteroid Rendezvous) will be the first Discovery mission flown. Mission costs are capped at $\$ 150$ million, excluding launch costs. NASA's plan is to cancel any mission that can not stay within the cost limit.

\section{Department of Defense}

The mean plane of Earth's orbit around the Sun

European Space Agency

The exact shape of the surface of a mirror or other optical component

Field of view: the region of sky visible to a detector at any one time

A type of $\mathrm{CCD}$ in which half the chip is masked. At the end of an exposure, the entire image is shifted rapidly to the masked half of the array. Then the masked portion is shifted, one column of pixels at a time, into the column register at the edge, and, after each 1-column shift, the register is slowly read out through an amplifier and other circuitry.

Frugal satellite: a generic satellite bus currently under development by Lockheed. It will be able to provide basic services, such as power, guidance, and communications, to a range of different payloads.

Fast steering mirror 
FTE

FWHM

GEO

Gigabyte

HACE

HgCdTe array

High-Z telescope

HST

Infrared astronomy

InSb array

IPSRU

IRAS
Full-time equivalent: a measure of the workload imposed by a particular activity

Full width at half maximum: a measure of the degree of concentration of light from a source produced by an optical system. It is equal to the width of a plot of intensity of light versus distance from the center of the image at a point where the intensity has fallen to half its maximum value.

Geostationary Earth orbit

One billion $\left(10^{9}\right)$ bytes: a unit of information used to describe quantities of data or the storage capacity of computers

Hubble Advanced Camera for Exploration: a third-generation instrument to be installed in the Hubble Space Telescope toward the end of this decade. This particular camera was recently chosen from among several proposals to build an Advance Camera $(\mathrm{AC})$ for the HST.

An array used for sensing near-infrared radiation. The photo-sensitive material is composed of an alloy of mercury ( $\mathrm{Hg}$ ), cadmium (Cd), and tellurium (Te). The spectral range extends from the red to an infrared cutoff that is adjustable in manufacture between 2.5 and 5 microns with trade-offs for poorer sensitivity in exchange for the wider spectral range. The arrays are addressable, storing signals in each pixel as a capacitor, from which the voltage is read. Thus the readout is fundamentally different from that of optical CCDs.

A proposed 4-meter space telescope optimized for cosmological observations

Hubble Space Telescope: a 2.4-meter-aperture, optical/ultraviolet telescope developed by NASA and the European Space Agency

The study of astronomical objects using intermediate-wavelength radiation to which the atmosphere is mostly opaque and the human eye insensitive. Humans sense infrared energy as heat. The infrared part of the electromagnetic spectrum generally corresponds to radiation with wavelengths from $1 \mu \mathrm{m}$ to $1000 \mu \mathrm{m}(1 \mu \mathrm{m}$ is one-millionth of a meter). Objects with temperatures around room temperature or lower emit most of their radiation in the infrared.

An array used for sensing near-infrared radiation. The photo-sensitive material is indium (In) antimonide ( $\mathrm{Sb}$ ). The spectral range extends from the far red to approximately 5 microns. Currently available InSb devices have greater sensitivity than $\mathrm{HgCdTe}$ devices when a response to 5 microns is required, but they also require operation at very low temperatures to achieve this sensitivity. Both technologies are still under active development.

Inertial Pseudo-Stellar Reference Unit: a device developed at Charles Stark Draper Laboratories to provide an inertially stable beam of light that can be tracked by an optical system in a spacecraft to maintain stable pointing

Infrared Astronomical Satellite: a cryogenically cooled, infrared satellite, developed as a cooperative program among the United States, the United Kingdom, and the Nether- 
IRTF

ISO

IUE

JFCs

Kuiper Airborne Observatory

Kuiper Disk

LAMP

Line-transfer CCD

LOS

Magnitude

mas

Molniya orbit

NASA lands, which between January and November 1983 made a survey of the entire sky at several different infrared wavelengths

Infrared Telescope Facility: a 3-meter telescope located on Mauna Kea, Hawaii, operated by NASA to study planets and other astronomical objects

Infrared Space Observatory: a 0.6-meter-aperture, cryogenically cooled, infrared space telescope to be launched by the European Space Agency in late 1995

International Ultraviolet Explorer: a joint U.S.-ESA-UK orbiting telescope to study ultraviolet radiation

Jupiter-family comets

A 0.9 -meter-diameter telescope for infrared and submillimeter observations that is carried above most of Earth's water vapor in a C-141 aircraft

The extension of the solar system beyond the Neptune-Pluto region to hundreds of astronomical units. Originally hypothesized by Edgeworth and by Kuiper to avoid a discontinuity in the surface density at Neptune-Pluto, this disk is now invoked as the source of the short-period comets of the inner solar system, all of which have dynamical lifetimes much less than the age of the solar system.

Large Active Mirror Program: a DOD-sponsored project at Itek Optical Systems to develop a 4-meter-aperture, actively controlled, segmented mirror for use in a spacebased, laser weapon

A type of CCD in which alternate rows of pixels are masked to facilitate rapid readout. To read out the array, each row of pixels is shifted (simultaneously) to the adjacent, masked row. The unmasked rows can then begin integrating again immediately, while the masked rows are read out slowly. The masked rows are read out along the row, one column of pixels at a time, to a column register at the end of the chip. After each shift along the rows by one column, the entire end-column register is shifted out sequentially to the read amplifier.

Large Optical Segment: a DOD-sponsored project at Itek Optical Systems to develop an 11-meter aperture, actively controlled, segmented mirror

A logarithmic unit of brightness for stars and other astronomical objects. Fainter stars have numerically larger magnitudes. The brightest stars, excluding the Sun, are about magnitude 0 ; the faintest star visible to the unaided eye is about magnitude 6. A star with $V=15$ is one-millionth as bright as the half-dozen brightest stars with $V=0$. Stars as faint as magnitude 28 can be seen with powerful terrestrial or spaceborne telescopes.

Milliarc second: one-thousandth of an arc second

A highly eccentric and inclined orbit with a nominal period of 12 hours used mainly by Russian telecommunication satellites

National Aeronautics and Space Administration 
NEO

New Millennium

NICMOS

NTOT

Occultation

Oort cloud

Optical astronomy

Parallax

Perigee

Perihelion

Pixel

Point-spread function

Polar Stratospheric

Telescope

Proton

Protoplanetary or protostellar disk

Protostar
Near-Earth orbit: object whose orbit brings it near Earth's orbit: specifically, certain asteroids and comets

A NASA program to develop and fly a series of ultra-lightweight spacecraft to demonstrate the advanced technology required for future space science missions

Near-Infrared Camera and Multi-Object Spectroscope: a second-generation instrument for the Hubble Space Telescope to be installed in 1997. This camera uses a HgCdTe array as its sensor.

New Technology Orbital Telescope

The obscuration of one celestial body by another of greater apparent diameter; especially the passage of the Moon in front of a star or planet, or the disappearance of a star behind an asteroid or comet.

A spherical cloud of comets having semimajor axes between 1,000 and 50,000 AU. Comets in this cloud, most of which have orbits with low eccentricity, can be sufficiently perturbed by passing stars or giant molecular clouds so that a fraction of them acquire orbits that take them within the orbits of Jupiter and Saturn.

The study of astronomical objects using light waves with wavelengths from about 0.3 to $1 \mu \mathrm{m}$. The human eye is sensitive to most of these wavelengths.

The apparent shift in position of a nearby object relative to a more distant object, as the observer changes position. Using basic trigonometry, it is possible to derive the distance of a star from its parallax as observed from opposite points on Earth's orbit.

The point at which a body in orbit around the Earth most closely approaches the Earth

The point at which a body in orbit around the Sun most closely approaches the Sun

The smallest element of a digital image. A typical image from a single chip in the Hubble Space Telescope is a square with $800 \times 800$ discrete pixels.

The variation of intensity with distance from the center of an image of a true point source. The PSF describes the observing instrument's effect on the light source. A detected image is a convolution of the true brightness distribution on the sky with the PSF of the instrument.

A proposed project in which a 4-meter telescope is suspended beneath a tethered aerostat

A family of large Russian launch vehicles

A disk of gas and dust surrounding a young star or protostar out of which planets may form

The earliest phase in the evolution of a star, in which most of its energy comes from the infall of material, or accretion, onto the growing star. A protostellar disk probably forms around the star at this time. 
PSF

$\mathrm{PtSi}$

Quadrature

Redshift

Refigure

Resolution

SDIO

SIRTF

STIS

Strehl ratio

STScI

Supernova

TGBNTOO

Titan IV

Type I Supernova

ULE

Van Allen Belts
Point-spread function

A platinum (Pt) silicide ( $\mathrm{Si}$ ) infrared array detector

The direction at right angles to the direction toward the Sun as seen from Earth. When another body of the solar system is at quadrature, its apparent angular motion is entirely due to its own motion. At any other point in the body's orbit, the motion of the Earth is also reflected in the apparent motion of the body.

The increase in the wavelength of a spectral line from an astronomical body relative to its value measured in a terrestrial laboratory. The wavelength shift is then given by the factor $(1+z)$. The redshift is usually interpreted as being due to the Doppler effect (motion away from the observer), and for nearby objects relative motions lead to both blueshifts and redshifts. At very large distances the redshift is interpreted in many cosmologies, but not all, as being due to the expansion of the universe.

Changing the shape of an optical surface

The ability of an instrument to discern fine detail

Strategic Defense Initiative Organization: the predecessor of BMDO

Space Infrared Telescope Facility: NASA's proposed 1-meter-aperture, cryogenically cooled, infrared space telescope

Space Telescope Imaging Spectrograph: a second-generation instrument that will be installed in the Hubble Space Telescope in 1997

A measure of the quality of an optical instrument equal to the ratio of the amplitude of the point-spread function to that of an equivalent, ideal instrument

Space Telescope Science Institute

An explosive death of a star whose tremendous energy output causes its expanding debris to glow brightly enough to be seen at extragalactic distances for weeks or months thereafter

\section{Task Group on BMDO New Technology Orbital Observatory}

A large U.S. launch vehicle used primarily by the Department of Defense

A type of supernova that occurs in a binary star system containing a white dwarf. All such events are believed to have the same intrinsic brightness and so can be used as distance indicators.

Ultra-low expansion: a type of glass ceramic used to make telescope mirrors

Zones of intense radiation surrounding Earth's mid-section, caused by charged particles trapped in Earth's magnetic field 
Wavefront

WFPC

WFPC2

YSOs

$z$

Zodiacal emission
A surface of constant phase, that is, an imaginary surface linking the crests or troughs of the rays of light from a common source

Wide Field/Planetary Camera: the primary camera used by the Hubble Space Telescope before its first servicing mission

Wide Field/Planetary Camera 2: the primary camera currently being used on the Hubble Space Telescope

Young stellar objects

Redshift: the increase in the wavelength of a spectral line from an astronomical body relative to its value measured in a terrestrial laboratory. The wavelength shift is then given by the factor $(1+z)$.

A faint glow caused by sunlight scattering off interplanetary dust near the plane of the ecliptic 

